\begin{abstract}
ATTWOOD, BRIAN CHRISTOPHER. Global phase diagram for monomer/dimer mixtures. (Under the direction of Carol K. Hall)

The objective of this thesis is to calculate the global phase diagram predicted by the Generalized Flory Dimer equation of state for mixtures of square-well monomers and dimers. Towards that goal, we first extend the Generalized Flory Dimer (GFD) theory for hard sphere monomer/dimer mixtures to square-well monomer/dimer mixtures. Theoretical predictions for the compressibility factor as a function of volume fraction are compared to discontinuous molecular dynamic simulation results on monomer/dimer mixtures at well depth ratios $0.5-1.5$ and dimer mole fractions $0.111-0.667$ and on monomers/8-mer mixtures at well depth ratios $0.5-1.5$. Agreement is found generally to be good and consistent with the agreement obtained when the GFD theory is applied to other square-well systems.
\end{abstract}

Next we calculate the GFD predicted global phase diagram for square-well monomer/dimer mixtures using a brute force method. The locus of critical points in the $P-T$ plane is calculated for a grid of points in the $\Lambda-\zeta$ plane, where $\zeta$ is a measure of the difference between the monomer and dimer well depths and $\Lambda$ is a measure of the strength of the attraction between monomers and dimers. Initially, the locus of critical points was calculated for 360 points in a square grid between the values of -0.9 and 0.9 for both $\zeta$ and $\Lambda$. Additional points were calculated as necessary to gain resolution in areas where more detail was needed.

The most significant features of the resulting global phase diagram are the absences of type IV and type VI behaviors. The absence of type VI behavior is not surprising because the existence 
of closed loop liquid-liquid immiscibility predicted by equations of state for molecules of spherical potentials is a matter of debate. The absence of type IV behavior is somewhat more unexpected because such behavior is found in most previous work on systems of spherical molecules. Although we performed extra calculations in the area in the $\zeta-\Lambda$ plane where type IV behavior should be exhibited, it is possible that our search was not intense enough to find the small region where type IV behavior might exist. The phase diagram is shifted towards the negative $\zeta$ and $\Lambda$ directions when compared to the van der Waals global phase diagram for equal diameter spherical molecules. The shift in the $\zeta$ direction can be attributed to the size difference between monomers and dimers. The shift in the $\Lambda$ direction implies that monomer/dimer systems have a greater tendency towards liquid-liquid immiscibility in our system than in monomer/monomer systems. 


\title{
GLOBAL PHASE DIAGRAM FOR MONOMER/DIMER MIXTURES
}

\author{
by \\ BRIAN CHRISTOPHER ATTWOOD
}

\author{
A thesis submitted to the Graduate Faculty \\ of North Carolina State University \\ in partial fulfillment of the \\ requirements for the Degree of \\ Master of Science
}

\section{Chemical Engineering}

Raleigh, NC 27695

2001

APPROVED BY:

Carol K. Hall

Richard J. Spontak

Chair of Advisory Committee

Robert E. Funderlic

Robert E. White 


\section{Biography}

The author was born in Troy, New York on April 27, 1975. He is the eldest son of Richard and Dorothea Attwood and has two brothers, Jason and Kristopher. He received a B.S. degree in Chemical Engineering from the State University of New York at Buffalo in May, 1997. In August, 1997, he was admitted to North Carolina State University to pursue graduate studies in Chemical Engineering. On June 21, 1998, he married Karen Elizabeth Zawoysky of Clifton Park, New York. 


\section{Acknowledgments}

Through their support and encouragement, many people have contributed to the preparation of this thesis. It gives me great pleasure to acknowledge them here.

First, I would like to thank my advisor, Professor Carol Hall. This thesis could not have been completed without the insights I gained from discussions we had on both technical and personal matters. Professor Hall is obviously very concerned about the professional and personal success of her students and I am grateful to be one of her students. I would also like to thank her for obtaining the funding necessary to support me and my research for the past several years.

I thank the GAANN Computational Sciences Fellowship Program of the U.S. Department of Education, the Office of Energy Research, Basic Sciences, Chemical Science Division of the U.S. Department of Energy, and the donors of the Petroleum Research Fund administered by the American Chemical Society for their financial support for the research included in this thesis.

I am grateful to Julie McCormick and Andrew Schultz for their parts in our joint effort to keep our computers running smoothly. Although at times our jobs are frustrating, we've learned that there is much wisdom in the saying that two (or three!) heads are better than one. I would also like to thank the other members of our research group, past and present, for their friendship and encouragement. To my other colleagues in the department with whom I have interacted, I thank you for the support you have shown my work and the enjoyment you given me socializing outside of the office.

To my family and friends, I am especially grateful for the love and encouragement that you have given me while in graduate school. Although I do not get to see you as much as I would like, I think of you often. To my parents, thank you for always expressing your confidence in my ability 
to succeed and pride in what I have accomplished. To Jason and Kris, even though you like to tease me about my extended schooling, I know that in your hearts you wish me well. Finally, to my wife, Karen, I thank you for being that one person I can always count on to be there for me in my defeats and my successes and who can always make me smile. 


\section{Contents}

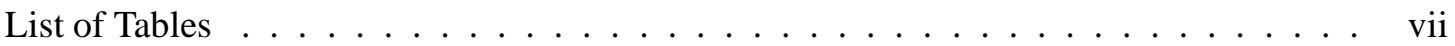

List of Figures $\ldots \ldots \ldots \ldots \ldots \ldots \ldots \ldots \ldots$ viii

1 Introduction 1

2 GFD equation of state for mixtures of square well chains and monomers 4

2.1 Review of Generalized Flory-Dimer theory for hard chain fluids $\ldots \ldots \ldots$

2.2 Review of Generalized Flory-Dimer theory for square-well chain fluids $\ldots \ldots \ldots$

2.3 Generalized Flory-Dimer theory for square-well chain/monomer mixtures . . . . 12

2.4 Comparison of GFD theory to molecular dynamic simulation . . . . . . . . . . 19

3 Calculation of Global Phase Diagram 21

4 Global Phase Diagram for Monomer/Dimer Mixtures 27

5 Summary 29

$\begin{array}{ll}\text { References } & 30\end{array}$

$\begin{array}{ll}\text { Appendices } & 42\end{array}$

$\begin{array}{lll}\text { Appendix A } & \text { Mixture Excluded Volume } & 43\end{array}$

$\begin{array}{lll}\text { Appendix B Code Listing } & 45\end{array}$ 
B.1 Code listing for calculating of the locus of critical points $\ldots \ldots \ldots \ldots$

B.1 Code listing for calculating coexistence points . . . . . . . . . . . 75 


\section{List of Tables}

1 Values of the density-independent coefficients of Equations 27, determined using Boublik's equation of state for mixtures $\ldots \ldots \ldots 33$

2 The shape parameter ratios, $\omega$ and $\psi$, and their derivatives, $\omega^{\prime}$ and $\psi^{\prime}$ with respect to $N_{i}$, in terms of species shape parameters and mixture shape parameters . . . . .

3 Species shape parameters for monomers and dimers determined from the segment radius,

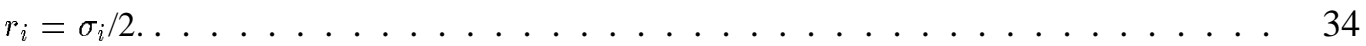

4 Coordination number model parameters, where $\sigma_{r}=\sigma_{22} / \sigma_{11} \ldots \ldots \ldots \ldots \ldots$. . . 34 


\section{List of Figures}

1 Comparison of GFD equation of state to molecular dynamics simulation results for mixtures of monomers and dimers at $\epsilon_{22} / \epsilon_{11}=0.5,1.0$ and 1.5 , equal segment diameters and equal volume fractions $x_{1} v_{1}=x_{2} v_{2}$ of the two species. . . . . .

2 Comparison of GFD equation of state to molecular dynamics simulation results for mixtures of monomers and dimers at $\epsilon_{22} / \epsilon_{11}=1.5$ and $x_{2}=0.111,0.333$, and

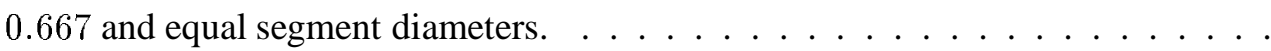

3 Comparison of GFD equation of state to molecular dynamics simulation results for mixtures of monomers and 8-mers at $\epsilon_{22} / \epsilon_{11}=0.5,1.0$, and 1.5 , equal segment diameters and equal volume fractions $x_{1} v_{1}=x_{2} v_{2}$ of the two species. . . . .

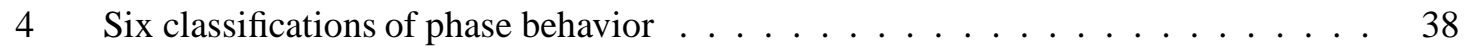

$5 \quad$ Schematic of a parametrized plot of $\mu_{1}$ vs. $\mu_{2} \ldots \ldots \ldots \ldots$

6 The global phase diagram predicted by the Generalized Flory Dimer theory for a mixture of square-well monomer and dimers. . . . . . . . . . . . . . . . . 40

7 A schematic of the global phase diagram predicted by the van der Waals equation of state for a mixture of equal-sized molecules as calculated by van Konynenburg

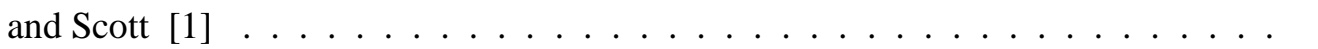




\section{Introduction}

Prediction of phase equilibria for multicomponent systems containing chain like molecules is important in a number of areas in industry, including oil refining, pharmaceutical purification, and the processing of many types of polymers, such as rubbers, plastics, and lubricants. Foreknowledge of the phase behavior of a solution containing chainlike molecules during processing allows us to induce or avoid phase separation as needed and hence is critical to successful design and operation of that process. One way to approach this problem that has become popular in recent years is to construct a global phase diagram that predicts the phase behavior expected for a particular equation of state. A global phase diagram gives a map of the types of phase behavior associated with a particular equation of state in intermolecular interaction parameter space. Using the knowledge embodied in a global phase diagram, a process engineer could tune a process, for example, with regards to the solvent strength, to select one type of phase behavior over another less-desirable phase behavior.

Applying phase equilibria conditions to a particular equation of state for the purpose of calculating phase coexistence is a technique that is well established. Usually such calculations are carried out for specific values of the molecular interaction parameters in order to predict the phase equilibrium of a particular system. A different approach was taken in the late sixties by van Konynenburg and Scott $[1,2]$ who chose to examine the phase behavior predicted for a binary mixture by the van der Waals equation of state over the entire range of interaction parameters. Their approach involved simultaneously solving the two equations, $\left(\partial^{2} G / \partial x_{i}^{2}\right)_{P, T}=0$ and $\left(\partial^{3} G / \partial x_{i}^{3}\right)_{P, T}=0$, that define a mixture critical point, where $G$ is the Gibb's free energy of mixing, $x_{i}$ is the mole fraction of component $i, P$ is the pressure, and $T$ is the temperature and hence calculating the locus of critical points as a function of composition, pressure, and temperature. The type of phase behavior could 
then be classified into one of six types based on the projection of the locus of critical points onto the $P-T$ plane.

For their analysis of mixtures of equal sized components, Scott and van Konynenburg [1,2] used two dimensionless parameters, $\zeta$ and $\Lambda$, which were a function of the attractive interaction parameters $a_{11}, a_{22}$, and $a_{12}$ between molecules of components 1 and 2 , where $\zeta=\left(a_{22}-a_{11}\right) /\left(a_{22}+\right.$ $\left.a_{11}\right)$ and $\Lambda=\left(a_{11}-2 a_{12}+a_{22}\right) /\left(a_{22}+a_{11}\right)$. For equal sized molecules, $\zeta$ is related to the differences in critical temperatures or pressures of the pure components and $\Lambda$ to the molar heat of mixing at $T=0$. By determining the locus of critical points for a range of these parameters, they were able to construct a global phase diagram which divides the $\zeta-\Lambda$ parameter space into regions of similar phase behavior. Their global phase diagram for the van der Waals equation of state accounts for five of the six types of phase behavior known to exist in nature. The only type of phase behavior not found by Scott and van Konynenburg was type VI, which is distinguished from the other types by a region of closed loop immiscibility. This was an important result because it showed that although the van der Waals equation of state is simplistic and quantitatively inaccurate for most fluids, it is able to predict much of the phase behavior exhibited in nature.

Since that landmark paper, a number of other groups have made similar explorations of the phase behavior for other equations of state. Furman et al. calculated the global phase behavior for a regular solution model of a three-component mixture that is nearly equivalent mathematically to a van der Waals model for binary mixtures with $b$ independent of concentration (implying the molecules are of equal size) and $a$ a quadratic function of composition [3]. They found significant agreement between the phase diagram they calculated and the one calculated by van Konynenburg and Scott for equal diameter molecules. Later Furman and Griffiths [4] revisited the global phase di- 
agram for the van der Waals model and discovered a heretofore unidentified "shield region", a small region containing several new classes of phase behavior that had not been pointed out previously by van Konynenburg and Scott.

Mazur and Boshkov [5-7] investigated the global phase behavior of binary Lennard-Jones fluid mixtures using a polynomial form for the equation of state that was proposed earlier and regressed to simulation data by Ree [8]. This was the first analysis of the phase behavior of a relatively modern equation of state that included a systematic look at the entire interaction parameter space. Mazur and Boshkov's papers revealed that the global phase behavior predicted for Lennard-Jones fluids was qualitatively similar to that of the van der Waals equation of state, except that Type VI phase behavior was also predicted. This result was controversial because closed loop immiscibility was thought to occur only in systems with strong directional bonding, e.g. hydrogen bonding. More recently, Yelash et al. $[9,10]$ presented evidence that strong directional forces may not be necessary for the appearance of closed loop immiscibility and that large deviations in the cross-interaction parameters from the averages of the pure substances interaction parameters are sufficient. Dieters and Pegg calculated the global phase diagram for the Redlich-Kwong equation of state [11] in 1989 and found that for equal sized molecules, the global phase diagram predicted by the Redlich-Kwong equation of state is similar to that found for the van der Waals fluid or the Lennard-Jones fluid. In this case however, no instances of Type VI behavior were found. As the difference in molecular size was increased, the topology of the global phase diagram changed considerably, with new sub-classes of phase behavior being predicted. Kraska and Dieters [12] analyzed the Carnahan-Starling-RedlichKwong equation of state a few years later in 1992. For equal sized molecules the results were again similar to those for the van der Waals equation of state. For molecules of different sizes, some unusual new phase behavior was found involving four-phase states and high density instabilities. 
One of the important results of this last study was that it showed that calculations based on a more "sophisticated," non-cubic equation of state yielded results that were, in the case of equal sized molecules, surprising similar to those found for cubic equations of state.

In this study, we consider the global phase diagram associated with the Generalized Flory Dimer equation of state for a binary mixture. The Generalized Flory Dimer theory evolved from work done in this laboratory to develop equations of state that predict the behavior of fluids containing chainlike molecules. Our approach has been to start with extremely simple models and then to systematically add more realistic features with the goal of thoroughly understanding the impact of each new feature on the fluid behavior. The generalized Flory theories were first introduced in 1986 by Dickman and Hall [13] who extended the probabilistic assumptions of the Flory lattice model to hard chains moving in continuous space. These theories were based on estimating the probability of inserting a chain molecule into a chain fluid and substituting this into the osmotic equation of state to find the compressibility factor of the chain fluid. The insertion probability estimate was built up in terms of the probability of inserting a monomer into a monomer fluid, which could be derived from an equation of state for that monomer fluid. Honnell and Hall [14] later refined the estimate for chain insertion to include terms related to the insertion of a monomer into a monomer fluid and a dimer into a dimer fluid culminating in the Generalized Flory-dimer (GFD) theory. Yethiraj and Hall $[15,16]$ extended the generalized Flory theory to square-well chains in 1991, adding a little more realism to what had previously been an equation of state for athermal chains. Our efforts over the past five years have been devoted to extending GFD to more realistic and/or complex fluids including: hard chain mixtures [17], alternating, block, and random heteronuclear square-well chain fluids [18-20], hard-chain networks [21] and gels [22]. One thing that Hall and coworkers did not do was derive a GFD expression for square well monomer/dimer mixtures. 
In this paper, we first derive the Generalized Flory Dimer theory for binary mixtures of square well monomers and dimers by extending the GFD theory for hard monomer/dimer mixtures to include attractions. This is accomplished in a manner similar to that used to extend GFD for pure hard chain fluids to pure square well chain fluids. The associated global phase diagram is found by calculating the locus of critical points at discrete points in interaction parameter space and then classifying the phase behavior at those points based on the resulting critical point locus topography. The calculation proceeds in a manner similar to that used by Van Konyenburg and Scott for the van der Waals equation of state, with the exception that numerical procedures are required to solve the governing equations. The locus of critical points for monomer/dimer mixtures of equal segment diameter was calculated at more than 100 discrete points in $\zeta-\Lambda$ space, with $\zeta$ and $\Lambda$ each ranging from - 0.9 to 0.9 . For this range of $\zeta$ and $\Lambda$, the value of $\epsilon_{11} / \epsilon_{22}$ ranged from 0.0526 to 19.0 and the value of $\epsilon_{12} /\left(\epsilon_{11} \epsilon_{22}\right)^{(1 / 2)}$ ranged from 0.1 to 4.36 .

Highlights of our results are the following. The Generalized Flory Dimer theory was found to produce good agreement with simulation for mixtures of monomers and dimers of equal segment diameter at varying temperatures, interaction strengths, and compositions. The agreement between simulation and theory improved when the dimer component in the mixtures was replaced with an 8mer. This result was to be expected based on an analysis by Kumar and coworkers [23] of the errors associated with the assumptions made in the derivation of the Generalized Flory dimer theories. The resulting global phase diagram was found to represent four of the six types of phase behavior found by Scott and van Konynenburg. Type IV and VI phase behaviors were absent from our phase diagram. Additional calculations in the areas where Type IV behavior was presumed to exist were not productive, leading us to believe that either we did not look hard enough, or that these types of phase behavior were not predicted by the GFD theory. Much like the Scott and van Konynenburg 
results for molecules of unequal diameter, the vertical line of symmetry in our $\zeta-\Lambda$ plot was found to be shifted towards negative values of $\zeta$. The horizontal boundary between regions that display liquid-liquid immiscibility, i.e. II and III, and those that do not, i.e. I and V, was shifted slightly in the negative $\Lambda$ direction.

The remainder of this paper is organized as follows. In Section 2 the Generalized Flory Dimer theory for hard chains and square-well chains is reviewed and the Generalized Flory Dimer theory for square-well chain/monomer mixtures is derived. Theoretical predictions for the compressibility factor are compared to simulation results. In Section 3 the method used to calculate the global phase diagram is described. In Section 4, the global phase diagram resulting from the GFD prediction for square-well monomer/dimer mixtures is presented. Section 5 concludes the paper with a brief summary. 


\section{GFD equation of state for mixtures of square well chains and monomers}

In this section, we derive the Generalized Flory-Dimer (GFD) equation of state for mixtures of square-well monomers and chains and compare the theory with simulation results. The starting point for the Generalized Flory-Dimer theory is the osmotic equation of state

$$
\frac{P(\eta, n)}{k T}=\frac{\eta}{v_{n}}\left[1-\ln p_{n}(\eta)\right]+\frac{1}{v_{n}} \int_{0}^{n} \ln p_{n}\left(\eta^{\prime}\right) d \eta^{\prime}
$$

which relates the $n$-mer pressure, $P$, to the $n$-mer insertion factor, $p_{n}$, the volume of one molecule, $v_{n}=(\pi / 6) n \sigma^{3}$, and the volume fraction, $\eta \equiv N v_{n} / V$, where $\sigma$ is the segment diameter. The insertion factor is defined as

$$
p_{n}(\eta)=\left\langle\exp \left(-\frac{U}{k T}\right)\right\rangle
$$

where $U$ is the potential energy change upon inserting a test chain of length $n$ into the fluid and the brackets indicate an average taken over all configurations of the test chain and the chains in the fluid. For hard chains, the insertion factor takes on values of 0 and 1 , leading to the interpretation of this parameter as the chain insertion probability. The insertion factor is also related to the chemical potential [23] by

$$
\frac{-\mu_{n}^{*}(\eta)}{k T}=\ln p_{n}(\eta)
$$

where $\mu_{n}^{*}(\eta)$ is the corrected residual chemical potential, defined as the difference between the residual chemical potential and the residual chemical potential at zero density. (The residual chemical potential is the difference between the real and ideal chemical potentials at a given set of conditions.) 
The compressibility factor, $Z$, can be expressed in terms of the chain insertion factor as

$$
Z \equiv \frac{P(\eta, n) v_{n}}{\eta k T}=\left[1-\ln p_{n}(\eta)\right]+\frac{1}{\eta} \int_{0}^{n} \ln p_{n}\left(\eta^{\prime}\right) d \eta^{\prime}
$$

Because equation (4) is thermodynamically exact, the only challenge in deriving a good equation of state for a chain fluid is to accurately estimate the insertion factors. The Generalized FloryDimer theory uses simple geometric arguments to estimate the chain insertion factors based on the insertion factors for monomers and dimers in monomer and dimer fluids; the latter are available because accurate equations of state have been developed for monomer and dimer fluids. The GFD approach has been applied to pure fluids of athermal and square-well chains and to mixtures of athermal chains. Below, we briefly review the GFD approach for pure hard chain fluids and pure square-well fluids, since this will be relevant to our derivation of the GFD theory for square-well chain/monomer mixtures.

\subsection{Review of Generalized Flory-Dimer theory for hard chain fluids}

For pure fluids, the GFD estimate for the probability of inserting a hard chain into a hard chain fluid is given by

$$
\begin{aligned}
p_{n}^{h s}(\eta) & =p_{1}^{h s}(\eta)\left[\frac{p_{2}^{h s}(\eta)}{p_{1}^{h s}(\eta)}\right]^{\left(v_{e}(n)-v_{e}(1)\right) /\left(v_{e}(2)-v_{e}(1)\right)} \\
& =p_{1}^{h s}(\eta)^{-Y_{n}} p_{2}^{h s}(\eta)^{Y_{n}+1}
\end{aligned}
$$


where $p_{1}^{h s}$ and $p_{2}^{h s}$ are the probability of inserting a hard monomer and a hard dimer, respectively, into a hard $n$-mer fluid, $v_{e}(n)$ is the volume excluded by an $n$-mer to a monomer, and

$$
Y_{n}=\frac{v_{e}(\eta)-v_{e}(2)}{v_{e}(2)-v_{e}(1)}
$$

Eq. (5) may be understood by imagining that the test chain is inserted into the chain fluid one segment at a time. The exact expression for the test chain insertion probability is given by

$$
p_{n}(\eta)=\left(\begin{array}{c}
\text { Probability } \\
\text { of } \\
\text { inserting } \\
\text { bead 1 }
\end{array}\right)\left(\begin{array}{c}
\text { Conditional } \\
\text { probability } \\
\text { of } \\
\text { inserting } \\
\text { bead } 2 \\
\text { given bead } 1
\end{array}\right)\left(\begin{array}{c}
\text { Conditional } \\
\text { probability } \\
\text { of inserting } \\
\text { bead } 3 \\
\text { given beads } \\
1 \text { and } 2
\end{array}\right) \cdots\left(\begin{array}{c}
\text { Conditional } \\
\text { probability } \\
\text { of inserting } \\
\text { bead } \mathrm{n} \\
\text { given beads } \\
1, \ldots, \mathrm{n}-1
\end{array}\right)
$$

The first term on the right hand side of Eq. (8) is the probability of inserting the first bead of the test chain, $p_{1}^{h s}$, and using mean field arguments may be approximated by the probability of inserting a monomer into a monomer fluid. The second term on the right hand side of Eq. (8) is the conditional probability of inserting the second bead of the test chain next to the already-inserted first bead, i.e. the probability of finding a hole in the chain fluid large enough to accommodate the extra excluded volume, $v_{e}(2)-v_{e}(1)$, required. This term may be approximated by $p_{2}^{h s} / p_{1}^{h s}$ where $p_{2}^{h s}$, is the probability of inserting a dimer into a chain fluid which is itself approximated by the probability of inserting a dimer into a dimer fluid. The third term is the conditional probability of inserting the third bead next to the second, i.e. the probability of finding a hole in the chain fluid large enough to accommodate the extra excluded volume, $v_{e}(3)-v_{e}(2)$, required. This term may be approximated 
by $\left[p_{2}^{h s} / p_{1}^{h s}\right]^{\left(v_{e}(3)-v_{e}(2)\right) /\left(v_{e}(2)-v_{e}(1)\right)}$, the conditional probability of inserting the second bead next to the first bead, raised to a power that corrects for the difference between the size of the hole required to place the third bead next to the second and that required to place the second bead next to the first. This type of reasoning is used repeatedly as beads are added to the chain, resulting in the chain insertion probability estimate given in Eq. (5).

Inserting Eq. (6) into the osmotic equation of state yields an expression for the compressibility factor of a hard $n$-mer fluid

$$
Z_{n}^{h s}(\eta)=\left(Y_{n}+1\right) Z_{2}^{h s}(\eta)-Y_{n} Z_{1}^{h s}(\eta)
$$

where $Z_{2}^{h s}(\eta)$ and $Z_{1}^{h s}(\eta)$ are the compressibility factors of hard dimer and monomer fluids. The monomer and dimer compressibility factors can be obtained from appropriate equations of state, such as the Carnahan-Starling [24] and Tildesley-Street [25] equations of state, respectively. The excluded volume of an $n$-mer, $v_{e}(n)$ can be approximated as a linear function of chain length [13]

$$
v_{e}(n) \approx v_{e}(3)+(n-3)\left[v_{e}(3)-v_{e}(2)\right]
$$

for $2 \leq n \leq 8$ and

$$
v_{e}(n) \approx v_{e}(1)[10.094+0.6374(n-15)]
$$

for $n>8$. [26] 


\subsection{Review of Generalized Flory-Dimer theory for square-well chain fluids}

The Generalized Flory-Dimer approach can also be applied to square-well chain fluids [15]. By using geometric and mean field arguments similar to those used in the development of a GFD equation of state for hard chain fluids, the insertion factor for a square-well chain in a square-well fluid, $p_{n}^{s w}(\eta)$ is given by

$$
\begin{aligned}
p_{n}^{s w}(\eta) & =p_{1}^{s w}(\eta)\left[\frac{p_{2}^{s w}(\eta)}{p_{1}^{s w}(\eta)}\right]^{\left(v_{e}(n)-v_{e}(1)\right) /\left(v_{e}(2)-v_{e}(1)\right)} \\
& =p_{1}^{s w}(\eta)^{-Y_{n}} p_{2}^{s w}(\eta)^{Y_{n}+1}
\end{aligned}
$$

where $p_{1}^{s w}$ is the insertion factor for a square-well monomer in a square-well monomer fluid, $p_{2}^{s w}$ is the insertion factor for a square-well dimer in a square-well dimer fluid, and $Y_{n}$ is the excluded volume ratio defined in Eq. (7). Note that this has the same functional form as the GFD expression for the insertion factor for a hard chain in a hard chain fluid (Eq. 5) except that the hard monomer and hard dimer insertion factors are replaced by square-well monomer and dimer insertion factors. When the above estimate for the square-well chain insertion factor is used in the osmotic equation of state, the resulting expression,

$$
Z_{n}^{s w}(\eta)=\left(Y_{n}+1\right) Z_{2}^{s w}(\eta)-Y_{n} Z_{1}^{s w}(\eta)
$$

is the Generalized Flory-Dimer equation of state for square-well chain fluids, where $Z_{2}^{s w}(n)$ and $Z_{1}^{s w}(n)$ are the compressibility factors for square-well dimer and monomer fluids, respectively. The square-well monomer and dimer compressibility factors can be obtained by a number of methods, such as perturbation theory [27, 28], coordination number models [29], or by using the RISM theory 
with the mean-spherical approximation closure $[15,30]$.

\subsection{Generalized Flory-Dimer theory for square-well chain/monomer mixtures}

The Generalized Flory-Dimer theory for binary mixtures is obtained by substituting suitable expressions for the insertion factors of each component in the so-called "alternate" form of the osmotic equation of state. The alternate form of the osmotic equation of state represents the process of inserting both species in the mixture simultaneously in proportion to their mole fractions, and is given by

$$
\begin{aligned}
\frac{P\left(\eta_{a}, \eta_{b}\right)}{k T}= & \frac{x_{a} \eta}{\bar{v}}\left[1-\ln p_{a}\left(\eta_{a}, \eta_{b}\right)\right]+\frac{x_{a}}{\bar{v}} \int_{0}^{\eta} \ln p_{a}\left(\eta_{a}^{\prime}, \eta_{b}^{\prime}\right) d \eta^{\prime} \\
& +\frac{x_{b} \eta}{\bar{v}}\left[1-\ln p_{b}\left(\eta_{a}, \eta_{b}\right)\right]+\frac{x_{b}}{\bar{v}} \int_{0}^{\eta} \ln p_{b}\left(\eta_{a}^{\prime}, \eta_{b}^{\prime}\right) d \eta^{\prime}
\end{aligned}
$$

where $x_{a}$ and $x_{b}$ are the mole fractions of species $a$ and species $b$ in the fluid, the volume fraction of the mixture is $\eta=(\pi / 6) \rho \sum_{i} x_{i} n_{i} \sigma_{i}^{3}$, and $\bar{v}=x_{a} v_{a}+x_{b} v_{b}$ is the mole fraction average of the volumes of species $a$ and $b$. The integration is performed at constant mole fraction of each species in the mixture so that

$$
\eta_{a}^{\prime}=x_{a} \frac{v_{a}}{\bar{v}} \eta^{\prime} ; \quad \eta_{b}^{\prime}=x_{b} \frac{v_{b}}{\bar{v}} \eta^{\prime}
$$

Before we derive the Generalized Flory-Dimer theory for chain/monomer mixtures, it is useful to introduce the following notation for the mixture insertion probabilities. The probability of inserting a molecule of species $i$ and length $n_{i}$ into a mixture at total volume fraction $\eta$ and composition $x_{a}$ containing species $a$ and $b$ chains of lengths $n_{a}$ and $n_{b}$, respectively, is denoted as $p\left(\eta, x_{a}\right)_{i_{n_{i}}}^{\left(n_{a}, n_{b}\right)}$. In general, species $i$ will be one of the mixture components, $a$ or $b$, but its length may not be the 
same as the length of the chains of component $a$ or $b$ in the mixture. The chain insertion factor will be written in terms of the more commonly used variables, total volume fraction and mole fraction, thus differing from the notation used in Eq. (16).

To apply the GFD theory to binary mixtures, it is necessary to estimate the insertion factors of each component in the fluid mixture. Consider a mixture of chains of component $a$ and monomers of component $b$. The insertion factor for chains of component $a$ takes the form

$$
p\left(\eta, x_{a}\right)_{a_{n_{a}}}^{\left(n_{a}, 1\right)} \approx\left(p\left(\eta, x_{a}\right)_{a_{1}}^{\left(n_{a}, 1\right)}\right)^{-\left(Y_{n_{a}}^{a}\right)_{m i x}} \times\left(p\left(\eta, x_{a}\right)_{a_{2}}^{\left(n_{a}, 1\right)}\right)^{\left[\left(Y_{n_{a}}^{a}\right)_{m i x}+1\right]},
$$

where $p\left(\eta, x_{a}\right)_{a_{1}}^{\left(n_{a}, 1\right)}$ is the factor for inserting a monomer of species $a$ into the chain/monomer mixture, $p\left(\eta, x_{a}\right)_{a_{2}}^{\left(n_{a}, 1\right)}$ is the factor for inserting a dimer of species $a$ into the chain/monomer mixture, and $\left(Y_{n_{a}}^{a}\right)_{\text {mix }}$ is the volume excluded in the mixture by a molecule of species $a$ and length $n_{a}$. Expressions for $\left(Y_{n_{a}}^{a}\right)_{m i x}$ are presented in Appendix A. Using arguments similar to those made in the GFD pure hard chain theory, the factor for inserting a monomer of species $a$ into the chain/monomer mixture is taken to equal the factor for inserting a monomer of species $a$ into the hypothetical monomer mixture created by breaking all of the chain bonds

$$
p\left(\eta, x_{a}\right)_{a_{1}}^{\left(n_{a}, 1\right)} \approx p\left(\eta, y_{a}\right)_{a_{1}}^{(1,1)}
$$

where $y_{a}$ is the mole fraction of species $a$ monomers in the hypothetical binary monomer mixture. Similarly the factor for inserting a dimer of species $a$ into the chain/monomer mixture is taken to equal the factor for inserting a dimer of species $a$ into the hypothetical dimer/monomer mixture 
creating by breaking every other bond along the chain

$$
p\left(\eta, x_{a}\right)_{a_{2}}^{\left(n_{a}, 1\right)} \approx p\left(\eta, y_{a}\right)_{a_{2}}^{(2,1)}
$$

where $y_{a}$ is now the mole fraction of species $a$ dimers in the hypothetical dimer/monomer mixture.

The factor for inserting a monomer of species $b$ into the chain/monomer mixture is taken to be equal to the factor for inserting a monomer of species $b$ into the hypothetical dimer/monomer mixture formed by breaking every other chain bond

$$
p\left(\eta, x_{a}\right)_{b_{1}}^{\left(n_{a}, 1\right)} \approx p\left(\eta, y_{a}\right)_{b_{1}}^{(2,1)}
$$

where $y_{a}$ is again defined to be the mole fraction of species a dimers in the hypothetical dimer/monomer mixture.

The species $a$ monomer (Eq. 18) and dimer (Eg. 19) insertion probabilities are substituted into Eq. (17) and then the species $a$ chain (Eq. 17) and species $b$ monomer (Eq. 20) insertion probabilities are substituted into the binary osmotic equation of state (Eq. 16) to obtain the GFD equation of state for chain/monomer mixtures 


$$
\begin{aligned}
Z^{G F D}\left(\eta, x_{a}\right)^{n_{a}, 1} \equiv & \frac{P\left(\eta, x_{a}\right) \bar{v}}{\eta k T} \\
= & x_{a}\left\{1-\left[\left(Y_{n_{a}}^{a}\right)_{m i x}+1\right] \ln p_{a_{2}}^{(2,1)}+\left(Y_{n_{a}}^{a}\right)_{m i x} \ln p_{a_{1}}^{(1,1)}\right\} \\
& +\frac{x_{a}}{\eta}\left[\left(Y_{n_{a}}^{a}\right)_{m i x}+1\right] \times \int_{0}^{\eta} \ln p_{a_{2}}^{(2,1)} d \eta^{\prime} \\
& -\frac{x_{a}}{\eta}\left(Y_{n_{a}}^{a}\right)_{m i x} \times \int_{0}^{\eta} \ln p_{a_{1}}^{(1,1)} d \eta^{\prime}+x_{b}\left[1-\ln p_{b_{1}}^{(2,1)}\right] \\
& +\frac{x_{b}}{\eta} \int_{0}^{\eta} \ln p_{b_{1}}^{(2,1)} d \eta^{\prime} .
\end{aligned}
$$

Eq. (21) can be written in a more compact form if we define a term, $\gamma\left(\eta, y_{a}\right)_{i_{n_{i}}}^{\left(n_{a}, 1\right)}$, similar to that in the single component osmotic equation of state

$$
\gamma\left(\eta, y_{a}\right)_{i_{n_{i}}}^{\left(n_{a}, 1\right)}=1-\ln p\left(\eta, y_{a}\right)_{i_{n_{i}}}^{\left(n_{a}, 1\right)}+\frac{1}{\eta} \int_{0}^{\eta} \ln p\left(\eta^{\prime}, y_{a}\right)_{i_{n_{i}}}^{\left(n_{a}, 1\right)} d \eta^{\prime}
$$

where the subscripts and superscripts on $\gamma$ are analogous to the subscripts and superscripts on the mixture insertion probabilities, and the inserted species $i$ is either a species $a$ chain of length $n_{a}$ or a species $b$ monomer. Using the $\gamma$ notation, the equation of state can then be written as

$$
Z^{G F D}=x_{a}\left[\left(\left(Y_{n_{a}}^{a}\right)_{m i x}+1\right) \gamma\left(\eta, y_{a}\right)_{a_{2}}^{(2,1)}-\left(\left(Y_{n_{a}}^{a}\right)_{m i x}\right) \gamma\left(\eta, y_{a}\right)_{a_{1}}^{(1,1)}\right]+x_{b} \gamma\left(\eta, y_{a}\right)_{b_{1}}^{(2,1)}
$$

The $\gamma$ 's can written in the form

$$
\gamma\left(\eta, y_{a}\right)_{i_{n_{i}}}^{\left(n_{a}, 1\right)}=\gamma^{h s}\left(\eta, y_{a}\right)_{i_{n_{i}}}^{\left(n_{a}, 1\right)}+\gamma^{s w}\left(\eta, y_{a}\right)_{i_{n_{i}}}^{\left(n_{a}, 1\right)}
$$

where $\gamma^{h s}$ is the hard sphere, or repulsive, contribution to the compressibility factor and $\gamma^{s w}$ is the 
square-well, or attractive, contribution to the compressibility factor. Eq. (23) can then be written as

$$
\begin{aligned}
Z^{G F D}= & \\
& x_{a}\left[\left(\left(Y_{n_{a}}^{a}\right)_{m i x}+1\right)\left\{\gamma^{h s}\left(\eta, y_{a}\right)_{a_{2}}^{(2,1)}+\gamma^{s w}\left(\eta, y_{a}\right)_{a_{2}}^{(2,1)}\right\}-\left(\left(Y_{n_{a}}^{a}\right)_{m i x}\right)\left\{\gamma^{h s}\left(\eta, y_{a}\right)_{a_{1}}^{(1,1)}\right.\right. \\
& \left.\left.+\gamma^{s w}\left(\eta, y_{a}\right)_{a_{1}}^{(1,1)}\right\}\right]+x_{b}\left[\left(\gamma^{h s}\left(\eta, y_{a}\right)_{b_{1}}^{(2,1)}+\gamma^{s w}\left(\eta, y_{a}\right)_{b_{1}}^{(2,1)}\right] .\right.
\end{aligned}
$$

Calculation of the hard sphere contribution to the compressibility factor is done using scaled particle theory (SPT). The derivation of $\gamma^{h s}$ for that equation of state was given by Wichert et al. [17] The general result is

$$
\begin{aligned}
\gamma\left(\eta, y_{a}\right)_{i}^{\left(n_{a}, n_{b}\right)} & = \\
- & {\left[1+\mathrm{W}\left(y_{a}\right)_{i}^{\left(n_{a}, n_{b}\right)}+\mathrm{Y}\left(y_{a}\right)_{i}^{\left(n_{a}, n_{b}\right)}+3 \mathrm{Z}\left(y_{a}\right)_{i}^{\left(n_{a}, n_{b}\right)}\right] \frac{\ln (1-\eta)}{\eta} } \\
+ & \left\{-\left[\mathrm{Y}\left(y_{a}\right)_{i}^{\left(n_{a}, n_{b}\right)}+3 \mathrm{Z}\left(y_{a}\right)_{i}^{\left(n_{a}, n_{b}\right)}+\mathrm{W}\left(y_{a}\right)_{i}^{\left(n_{a}, n_{b}\right)}\right]\right. \\
& +\eta\left[-\mathrm{X}\left(y_{a}\right)_{i}^{\left(n_{a}, n_{b}\right)} / 2+(5 / 2) \mathrm{Y}\left(y_{a}\right)_{i}^{\left(n_{a}, n_{b}\right)}+(15 / 2) \mathrm{Z}\left(y_{a}\right)_{i}^{\left(n_{a}, n_{b}\right)}\right. \\
& \left.+3 \mathrm{~W}\left(y_{a}\right)_{i}^{\left(n_{a}, n_{b}\right)}\right]-\eta^{2}\left[\mathrm{X}\left(y_{a}\right)_{i}^{\left(n_{a}, n_{b}\right)} / 2+(5 / 2) \mathrm{Y}\left(y_{a}\right)_{i}^{\left(n_{a}, n_{b}\right)}\right. \\
& \left.\left.+(11 / 2) \mathrm{Z}\left(y_{a}\right)_{i}^{\left(n_{a}, n_{b}\right)}+3 \mathrm{~W}\left(y_{a}\right)_{i}^{\left(n_{a}, n_{b}\right)}\right]+\eta^{3} \mathrm{~W}\left(y_{a}\right)_{i}^{\left(n_{a}, n_{b}\right)}\right\}(1-\eta)^{-3},
\end{aligned}
$$

where the coefficients $W, X, Y$, and $Z$ are determined from SPT and are listed in Table 1 . The shape parameter ratios and shape parameters required to calculate $W, X, Y$, and $Z$ can be found in Tables 2 and 3 , respectively.

The square well contribution to the compressibility factor of the mixture, $\gamma^{s w}\left(\eta, y_{a}\right)_{i_{n_{i}}}^{\left(n_{a}, 1\right)}$, is derived from a local composition theory. Local composition theory predicts the coordination 
numbers of the various species in the mixture, which in turn can be used to calculate insertion factors for the two species. We chose to use the form of local composition theory introduced by Lee and Chao [29] and to apply the high density correction of Vimalchand et al.[31]. The empirical parameters appearing in this equation are fit to our own simulation data.

The coordination number $z_{i j}$ represents the number of molecules of component $j$ located inside the energy well of a square-well molecule of component $i$. It is related to the radial distribution function, $g_{i j}(r)$, by

$$
z_{i j}=\frac{4 \pi N_{j}}{V} \int_{\sigma_{i}}^{\lambda_{i} \sigma_{i}} g_{i j}(r) r^{2} d r
$$

where $\lambda_{i}$ is the ratio of the outside diameter of the SW potential to the hard-core diameter of component $i$ and is set equal to 1.5. The model of Lee and Chao defined $z_{i j}$ to be

$$
z_{i j}=\frac{V_{s, i j} Z_{t} \rho_{j} \Omega_{i j}}{1+\rho \sigma_{i j}^{3}[\Omega-1]}
$$

where,

$$
\begin{gathered}
\Omega_{i j}=\exp \left(\alpha \epsilon_{i j} / k T\right), \\
\Omega=\sum_{i} \sum_{j} x_{i} x_{j} \Omega_{i j}, \\
\alpha=1-\frac{\rho^{*}}{\sqrt{2}}, \\
\rho^{*}=\rho_{1} \sigma_{1}^{3}+\rho_{2} \sigma_{2}^{3}, \quad \rho_{i}=x_{i} \rho
\end{gathered}
$$

and $V_{s, i j}\left(\equiv \frac{4}{3} \pi\left(\lambda^{3}-1\right) \sigma_{i j}\right)$ is the volume of the coordination shell. The inter-species hard-core diameter, $\sigma_{12}$, is defined as $\frac{1}{2}\left(\sigma_{11}+\sigma_{22}\right)$, while the inter-species well-depth, $\epsilon_{12}$, normally defined 
to be $\left(\epsilon_{11} \epsilon_{22}\right)^{1 / 2}$, is allowed to vary. The parameter $Z_{t}$ differs from that of Lee and Chao due to the application of the high density correction of Vimalchand et al and is defined as

$$
Z_{t}=A+B \rho^{*}-C\left(\rho^{*}\right)^{4}
$$

where the coefficients $A, B$, and $C$ depend on the types of components in the mixture and can be found in Table 4. Because Vimalchand et al. did not apply the high density correction to the model of Lee and Chao, the coefficients in Table 4 were regressed from a least squares fit of our own simulation data of sqaure-well monomers and dimers.

Once the $z_{i j}$ 's have been calculated, the internal energy of the square-well fluid, $U$, is determined by

$$
U=-\sum_{i} \sum_{j} \frac{N_{i}}{2} z_{i j} \epsilon_{i j}
$$

The Helmholtz energy, $A$, is obtained by integrating the equation

$$
\left[\frac{\partial(A / T)}{\partial(1 / T)}\right]_{N, V}=U
$$

to get an expression for the Helmholtz energy,

$$
A=T \int_{0}^{1 / T} U d\left(1 / T^{\prime}\right)
$$

Because the Helmholtz energy as calculated above is dependent on the overlap of the square wells, the Helmholtz free energy at ideal gas conditions and at zero density will both be equal to zero. For this reason, the chemical potential so calculated will be equal to the corrected residual 
potential and can be set equal to the insertion factor.

To calculate the chemical potential of species $i$ from the Helmholtz energy, we differentiate with respect to $N_{i}$, the number of molecules of species $i$. However, for our purposes the chemical potential can be expressed more conveniently in terms of a partial derivative with respect to $x_{i}$

$$
\begin{aligned}
\ln p\left(\eta, y_{a}\right)_{i_{n_{i}}} & =\left(\frac{\partial A}{\partial N_{i}}\right)_{T, V, N_{j \neq i}} \\
& =A-x_{i}\left(\frac{\partial A}{\partial x_{i}}\right)_{T, V, x_{j \neq i}} \\
& =T \int_{0}^{1 / T}\left[U-x_{i}\left(\frac{\partial U}{\partial x_{i}}\right)_{T, V, x_{j \neq i}}\right] d(1 / T)^{\prime} .
\end{aligned}
$$

The resulting expressions for $\ln p\left(\eta, y_{a}\right)_{i_{n_{i}}}$ can then be substituted in Eq. (22) to calculate

$\gamma^{s w}\left(\eta, y_{a}\right)_{i_{n_{i}}}^{\left(n_{a}, 1\right)}$ for $i_{n_{i}}=a_{1}, a_{2}, b_{1}$ which, along with the expressions for $\gamma^{h s}\left(\eta, y_{a}\right)_{i_{n_{i}}}^{\left(n_{a}, 1\right)}$ for $i_{n_{i}}=a_{1}, a_{2}, b_{1}$ derived previously, can be used to calculate the compressibility factor of the squarewell chain/monomer mixture by Eq. (23).

\subsection{Comparison of GFD theory to molecular dynamic simulation}

In this section we compare the Generalized Flory Dimer equation of state derived in Section 2.3 with discontinuous molecular dynamics simulations of monomer-dimer and monomer-chain mixtures. Figure 1 compares the GFD theory predictions and discontinuous molecular dynamics simulation values for the compressibility factor as a function of the volume fraction for mixtures of square-well monomers (species 1) and dimers (species 2) of equal segment diameter $\sigma_{11}=\sigma_{22}$, equal volume fraction in the mixture $x_{1} v_{1}=x_{2} v_{2}$, temperature $T^{*} \equiv k T / \epsilon=2.0$, and well depth parameter ratios $\left(\epsilon_{11} / \epsilon_{22}\right)$ ranging from 0.5 to 1.5 . Agreement between theory and simulation is quite good, 
although the GFD theory tends to slightly overpredict the compressibility over the entire range of volume fraction.

Figure 2 compares the GFD theory predictions and simulation values for the compressibility factor of mixtures of square-well monomers and dimers of equal segment diameter at $T^{*}=2$, $\left(\epsilon_{11} / \epsilon_{22}\right)=1.5$, and $x_{2}=0.111,0.333$, and 0.667 . For $x_{2}=0.111$, the agreement is quite good except at the highest volume fraction. As the mole fraction of dimer increases, the agreement between theory and simulation became worse for the mixtures in the mid-range of total volume fraction, suggesting that some improvement in accounting for the effect of the presence of dimer species on the compressibility factor may be needed.

Figure 3 compares the GFD theory for the compressibility factor to discontinuous molecular dynamics simulation data for mixtures of square-well monomers (species 1) and 8-mers (species 2) of equal segment diameter $\sigma_{11}=\sigma_{22}$, equal volume fraction $x_{1} v_{1}=x_{2} v_{2}, T^{*}=2.0$, and well depth parameter ratios $\left(\epsilon_{22} / \epsilon_{22}\right)$ ranging from 0.667 to 2.0. Agreement between the theory and simulation is good for all well depth parameter ratios in this case. It is not surprising that the agreement between theory and simulation increases as the length of the "chain" component increases based on the findings of Kumar, et al [23] concerning the accuracy of the various predictions made in the Generalized Flory Dimer theory. They found that although the estimate for the probability of inserting the first bead is not very accurate, this tended to be offset by errors in estimating the probability of inserting the third through $n$th segments. Thus, it might be expected that the Generalized Flory Dimer theory would make more accurate predictions for mixtures of monomers and chains than for monomers and dimers. 


\section{Calculation of Global Phase Diagram}

In order to construct a global phase diagram it is necessary to determine the boundaries between regions of different types of phase behavior. Although, there are several of ways to do this $[1,11]$, the most straightforward way is to determine the type of phase behavior exhibited on a grid of points in interaction parameter space. The interaction parameters generally depend on the same-species interaction energies, $\epsilon_{i i}$, and on the inter-species interaction energies, $\epsilon_{i j}$. As is the case in most previous studies of global phase behavior for species of equal hard-core diameter, we will use the two parameters

$$
\begin{gathered}
\zeta=\frac{\epsilon_{22}-\epsilon_{11}}{\epsilon_{22}+\epsilon_{11}} \\
\Lambda=\frac{\epsilon_{11}-2 \epsilon_{12}+\epsilon_{22}}{\epsilon_{22}+\epsilon_{11}} .
\end{gathered}
$$

to define the parameter space for our global phase diagram. The type of phase behavior exhibited at each point $\zeta, \Lambda$ was determined by first calculating the locus of critical points. The phase behavior could then be classified based on the projection of the locus of critical points onto the $P-T$ plane.

Figure 4 shows characteristic projections of the locus of critical points onto the $P-T$ plane for Types I-VI phase behavior [11]. The thick solid lines represent the boiling point curves of the pure fluids, the thin solid lines, if present, represent the temperature as a function of pressure at three phase coexistence, and the dashed line is locus of critical points for the mixtures.

The loci of critical points can be calculated by solving the following equations for the critical temperature and pressure over a range of compositions from pure component one to pure component 
two

$$
\left(\frac{\partial^{2} G}{\partial x^{2}}\right)_{P, T}=\left(\frac{\partial^{3} G}{\partial x^{3}}\right)_{P, T}=0
$$

where $G$ is the Gibbs free energy of the mixture. However, it is not convenient to calculate the derivatives of the Gibbs free energy at constant pressure using an equation of state explicit in pressure. This hurdle can be overcome by using a Legendre transformation to change the thermodynamic equations defining a critical point Eq. (36) from functions of the Gibbs free energy to functions of the Helmholtz free energy, leading to the following critical point equations

$$
\begin{gathered}
A_{2 V} A_{2 x}-A_{V x}^{2}=0 \\
A_{3 V} A_{2 x}^{2}-3 A_{2 V x} A_{V x} A_{2 x}+3 A_{V 2 x} A_{V x}^{2}-A_{3 x} A_{2 V} A_{V x}=0
\end{gathered}
$$

where,

$$
A_{m V n x} \equiv\left(\frac{\partial^{n+m} A}{\partial^{m} V \partial^{n} x}\right)_{T}
$$

The Helmholtz free energy can be expressed in terms of the mixture compressibility factor, packing fraction, and mole fraction as [32]

$$
\frac{A}{R T}-\frac{A^{\circ}}{R T}=\int_{0}^{\eta} \frac{Z-1}{\eta^{\prime}} d \eta^{\prime}-\ln \frac{V}{V^{\circ}}+\sum_{i} x_{i} \ln x_{i}
$$

where $A^{\circ}$ and $V^{\circ}$ are reference state conditions that are independent of volume and composition. Thus, when the partial derivatives in Eqs.(37) and (38) are performed $A^{\circ}$ and $V^{\circ}$ will drop out of the resulting expressions.

The compressibility factor used in equation Eq. (40) was calculated using the Generalized 
Flory Dimer equation of state for square-well chain/monomer mixtures derived in Section 2.3. Eqs. (37) and (38) were then solved numerically using Newton's method [33] for the critical temperature and volume at fixed values of $\zeta, \Lambda$, and composition. Newton's method is actually a function minimization method that can be used to solve a system of equations by constructing a function equal to the sum of the squares of the left hand sides of those equations. Given an initial estimate of the solution to the system of equations, Newton's method uses information from the Jacobian matrix to determine the direction in variable space in which the function would be minimized. In addition to providing a direction in which to take the next estimate, the method also provides the size of the step that should be taken in that direction. Rather than adopting this step size, we chose to find the minimum of the function along the Newton direction, because this could potentially lead to quicker convergence. At each iteration step the derivatives in Eqs. (37) and (38) and the Jacobian matrix were calculated numerically. Given a sufficient number of iterations the method was said to have converged when the value of the function went below a given tolerance. Once the critical volume and temperature were calculated, the pressure was determined from the compressibility factor in Eq. (23).

Because of the existence of multiple solutions, an accurate initial estimate was needed to ensure that the iteration procedure converges to the desired root. This requirement was circumvented in the following manner. The calculations were typically started at a composition close to zero or one. This was done because the values of the critical pressure and temperature of a nearly pure fluid should be close to the values of the critical pressure and temperature of a pure fluid, the critical point of which can be easily calculated. Convergence towards the desired solution was typically rapid due the proximity of the initial guess. The composition could then be incremented further and the solution for the old composition used as the initial guess for the new composition. 
The procedure described above worked well in many cases, except when the locus of critical points did not connect with either of the pure component critical lines, such as occurs for example in liquid-liquid equilibrium. In these cases, it was was necessary to find an alternative means of generating an initial guess for the solution. To accomplish this, we calculated phase diagrams in the $T-x$ plane and from them was able to make accurate initial estimates for points on the sections of the locus of critical points mentioned above. For example, for a given set of $\Lambda$ and $\zeta$, the upper critical solution temperature (UCST) obtained from a phase diagram in the $T-x$ plane could be used as a starting point for finding the rest of the UCST branch of the locus of critical points in the $P-T$ plane.

Calculating phase diagrams in the $T-x$ plane involves finding coexistence points at a number of temperatures at a given pressure. At each temperature for a given pressure, the coexistence points were found by solving the standard equations that define equilibrium between two phases $\alpha$ and $\beta$, namely $T^{\alpha}=T^{\beta}, P^{\alpha}=P^{\beta}, \mu_{i}^{\alpha}\left(T^{\alpha}, P^{\alpha}, x_{i}^{\alpha}\right)=\mu_{i}^{\beta}\left(T^{\beta}, P^{\beta}, x_{i}^{\beta}\right)$,where $\mu_{i}^{j}$ is the chemical potential of component $i$ in phase $j$. Since the first two conditions are trivial, the calculation primarily involves finding the composition of each phase to satisfy the third condition.

The calculations of the compositions of the phases in equilibrium began by finding the volume as a function of composition at the given temperature and pressure according to Eq. (23). This required an iterative solution because Eq. (23) is implicit in volume and one or three solutions for the volume at each value of composition could be found depending on the composition, temperature, and pressure. Our approach was to fix the composition and then search for solutions while incrementing the volume upward from nearly close-packed until we were satisfied that all the solutions had been found. Solutions that were determined to be mechanically unstable based on the slope of 
the pressure versus volume curve at that point were thrown out. Once this was accomplished for a number of compositions ranging from one pure component to another, the Gibbs free energy was calculated at each value of the volume and composition according to

$$
\frac{G}{R T}-\frac{G^{\circ}}{R T}=\int_{0}^{\eta} \frac{Z-1}{\eta^{\prime}} d \eta^{\prime}-\ln \frac{V}{V^{\circ}}+Z-1+\sum_{i} x_{i} \ln x_{i}
$$

where $G^{\circ}$ and $V^{\circ}$ are reference state variables that could be set to 0 and 1 respectively and subsequently neglected. At this point, only the most stable value of volume (the one resulting in the lowest Gibbs free energy) was chosen for each composition. Once the Gibbs free energy had been calculated as a function of composition and volume over the entire range of composition, the chemical potential at $x_{i}$ was then calculated by

$$
\mu_{i}=G-x_{i}\left(\frac{\partial G}{\partial x_{i}}\right)_{T, P}
$$

The derivative appearing above was calculated numerically from the values calculated by Eq. (41).

The final step in the calculation involved finding values of the composition at which $\mu_{i}^{\alpha}=$ $\mu_{i}^{\beta}$. The algorithm essentially boiled down to plotting a parametrized curve of $\mu_{1}$ versus $\mu_{2}$ with composition as the parameter; this is represented schematically in Figure 5. Point A in Figure 5 represents a point at which $\mu_{1}$ and $\mu_{2}$ have the same value at two different values of composition. Three phase coexistence would be represented in Figure 5 by having the solid line pass through point A three times. To find point A numerically, we first calculated the slope and intercept in the $\mu_{1}-\mu_{2}$ plane for the line going from each value of composition to the successive value of composition, for example, from 1 to 2, 2 to 3, etc. in Figure 5. We then determined which line 
segments intersected each other. For example the line segment connecting points 3 and 4 intersects the line segment connecting points 5 and 6 . When such an intersection was found we interpolated along the $\mu_{1}$ versus $\mu_{2}$ line to arrive at the values of composition for the coexisting phases.

With this additional tool we calculated the locus of critical points for 360 points in $\zeta-\Lambda$ space, with grid values of each parameter ranging from -0.9 to 0.9 , in steps of 0.1 units in each diretion. As the loci were calculated the type of phase behavior exhibited was recorded and categorized according to Figure 4. After this initial grid search was completed approximately 100 additional calculations were performed along the boudary between Type V and I and between Types II and III. These calculations were done every 0.1 units in the $\Lambda$ and $\zeta$ directions along the boundary at a point midway between the points in our initial grid that bracketed the boundary at that point. The effect was such that the resolution along the boundary was effectively doubled. 


\section{Global Phase Diagram for Monomer/Dimer Mixtures}

Figure 6 shows the global phase diagram that we calculated based on the Generalized Flory Dimer equation of state for a mixture of square-well monomers and dimers. In the figure, the solid lines indicate the boundaries between different phase behavior types, while the dashed lines indicate boundaries between azeotropic and non-azeotropic behavior of a single type. Due to the finite number of calculations that were performed to locate the boundaries of the various regions, the boundaries in Figure 6 do not represent the exact boundary between any two regions. Because of the size of the grid used, the deviation between the actual boundary and that shown in Figure 6 should be no greater than 0.05 units in the $\Lambda$ or $\zeta$ directions.

Figure 7 is a schematic of the global phase diagram predicted for the van der Waals equation of state as calculated by van Konynenburg and Scott [34]. Comparison of Figures 6 and 7 reveals the absence of Type IV and Type VI behaviors on Figure 7. The absence of Type IV behavior is surprising because it had been described in most of the papers cited earlier in our literature survey, albeit normally in a rather restricted region of the global phase diagram. In the case of the CarnahanStarling-Redlich-Kwong equation of state, Kraska and Deiters [12] found that for molecules of different sizes, the region of Type IV behavior was nearly indistinguishable from the boundaries surrounding it. A possible explanation for its absence in our global phase diagram is that our search grid may not have been fine enough to find the Type IV. An additional 20 calculations were performed in the regions where Type IV phase behavior was expected $(\zeta=-0.75$ to $-0.9, \Lambda=-0.1$ to 0.1 and $\zeta=0.45$ to $0.6, \Lambda=-0.1$ to 0.1 , in steps of 0.05 units in both directions), but Type IV phase behavior was not observed. There are two possible explanations as to why no Type VI or other closed loop behavior was detected. The first is that our grid search may not have been fine enough to detect 
Type IV behavior; support for this view came from the fact that closed loop behavior is typically represented by an area on the global phase diagram even smaller than that of Type VI behavior, and is normally located in the "shield" region. We performed an additional 25 calculations in this region $(\Lambda=0.05$ to $0.25, \zeta=0.55$ to 0.75 in steps of 0.05 in each direction). The second possible explanation is that the Generalized Flory Dimer theory simply does not predict Type VI, or closed loop liquid-liquid immiscibility, for this type of system. As mentioned in Section 1, there has been an ongoing debate as to whether or not closed loop liquid-liquid immiscibility exists in systems of spherical molecules or if it is a mathematical artifact of approximate equations of state.

Another difference between the GFD and van der Waals global phase diagram is that the GFD diagram is not symmetric around the $\zeta=0$ axis. In light of the fact that van Konynenburg and Scott saw a similar shift for the case of unequal sized spheres, this result is not surprising. Since the molecules are not symmetrical they do not interact "symmetrically" and the global phase diagram is shifted accordingly. The final feature worth noting is that the boundary between regions with three phase coexistence and those without (the $\Lambda=0$ axis in the van der Waals case) is shifted slightly in the negative $\Lambda$ direction. This indicates that for monomer/dimer mixtures there is tendency towards liquid-liquid immiscibility when there are favorable energetic conditions for miscibility, indicating that entropic effects may play a role. Similar shifts were seen in the global phase diagrams predicted for other equations of state $[11,12]$, so this result has some precedence. 


\section{Summary}

We have presented a derivation of the Generalized Flory Dimer theory for monomer/chain mixtures which accurately predicts compressibilty factors compared to simulation data for mixtures at chain lengths 2 and 8 and varying compositions and ratios of $\epsilon_{22} / \epsilon_{11}$. The theory requires as input accurate equations of state for hard sphere monomer/dimer mixtures and for square-well monomer/dimer mixtures; these were obtained from scaled particle theory and the local composition model, respectively.

We have also presented the global phase diagram for mixtures of square-well monomer and dimers predicted by the Generalized Flory Dimer equation of state. The values of $\Lambda$ and $\zeta$ covered in the global phase diagram each ranged from -1.0 to 1.0. The phase diagram was constructed from critical point loci calculations at over 360 grid points in the $\Lambda-\zeta$ plane, followed by many additional calculations performed during concentrated searches. While four of the six phase behavior types found in nature were represented in the global phase diagram, Types IV and VI were absent. While the absence of type VI behavior was not surprising due to the current debate over its physicality in systems of spherical potentials, the absence of Type IV behavior was unexpected. Due to the limited resolution of our grid search it is unclear whether these regions are indeed not present or are simply too small for us to find. Other features of note were the shifting of the major topographical features of the global phase diagram towards the negative $\zeta$ and $\Lambda$ directions when compared to the van der Waals global phase diagram for equal diameter molecules. The former was expected based on the results of van Konynenburg and Scott. The latter implies that there is a factor working against the favorable interspecies interactions that leads to liquid-liquid immiscibility. 


\section{References}

[1] van Konynenburg, P. H. and Scott, R. L. , Discuss Faraday Soc., 49, 87 (1970).

[2] van Konynenburg, P. H. . PhD thesis, University of California, Los Angeles, (1968).

[3] Furman, D., Dattagupta, S. , and Griffiths, R. B., Phys. Rev. B, 15, 441 (1977).

[4] Furman, D. and Griffiths, R. B., Phys. Rev. A, 17, 1139 (1978).

[5] Mazur, V. A., Boshkov, L. Z., and Fedorov, V. B., Dokl. Akad. Nauk SSSR, 282, 137 (1985).

[6] Boshkov, L. Z. and Mazur, V. A., Russ. J. Phys., 60, 16 (1986).

[7] Boshkov, L. Z. , Dokl. Akad. Nauk SSSR, 294, 901 (1987).

[8] Ree, F. H. , J. Chem. Phys., 73, 5401 (1980).

[9] Yelash, L. V. and Kraska, T. , Phys. Che. Chem. Phys., 1, 307 (1999).

[10] Yelash, L. V., Kraska, T. , and Deiters, U. K. , J. Chem. Phys., 110, 3079 (1999).

[11] Deiters, U. K. and Pegg, I. L. , J. Chem. Phys., 90, 6632 (1989).

[12] Kraska, T. and Deiters, U. K. , J. Chem. Phys., 96, 539 (1992).

[13] Dickman, R. and Hall, C. K. , J. Chem. Phys., 85, 4108 (1986).

[14] Honnell, K. G. and Hall, C. K. , J. Chem. Phys., 90, 1841 (1989).

[15] Yethiraj, A. and Hall, C. K. , J. Chem. Phys., 95, 8494 (1991).

[16] Hall, C. K. , Yethiraj, A., and Wichert, J. M. , Fluid Phase Equil., 83, 313 (1993). 
[17] Wichert, J. M. , Gulati, H. S. , and Hall, C. K. , J. Chem. Phys., 104, 5220 (1996).

[18] Wichert, J. M. , Gulati, H. S. , and Hall, C. K. , J. Chem. Phys., 105, 7669 (1996).

[19] Gulati, H. S. and Hall, C. K. , J. Chem. Phys., 107, 3930 (1997).

[20] Gulati, H. S. and Hall, C. K. , J. Chem. Phys., 108, 7478 (1998).

[21] Kenkare, N. R. , Hall, C. K. , and Hall, S. A. , J. Chem. Phys., 110, 7556 (1999).

[22] Kenkare, N. R. , Hall, C. K. , and Hall, S. A. , J. Chem. Phys., 113, 404 (2000).

[23] Kumar, S. K. , Szleifer, I. , Hall, C. K. , and Wichert, J. M. , J. Chem. Phys., 104, 9100 (1996).

[24] Carnahan, N. F. and Starling, K. E. , J. Chem. Phys., 51, 635 (1969).

[25] Tildesley, D. J. and Street, W. B. , Mol. Phys., 41, 85 (1980).

[26] Denlinger, M. A. and Hall, C. K., Mol. Phys., 71, 541 (1990).

[27] Barker, J. A. and Henderson, D. , J. Chem. Phys., 47, 2856 (1967).

[28] Chang, J. and Sandler, S. I. , Mol. Phys., 81, 745 (1994).

[29] Lee, R. J. and Chao, K. C. , Mol. Phys., 61, 1431 (1987).

[30] Smith, W. R., Henderson, D. , and Tago, Y., J. Chem. Phys., 64, 5026 (1976).

[31] Vimalchand, P. , Thomas, A. , Economou, I. G. , and Donohue, M. D. , Fluid Phase Equil., 73, 39 (1992).

[32] Modell, M. and Reid, R. C. , Thermodynamics and its applications. Prentice-Hall, (1983).

[33] Press, W. H., Numerical Recipes in FORTRAN 77. Cambridge University Press, (1996). 
[34] van Konyenburg, P. H. and Scott, R. L., Phil. Trans. Roy. Soc. London (A), 298, 495 (1980).

[35] Boublik, T. M. , Mol. Phys., 27, 1415 (1974).

[36] Dodd, L. R. and Theodorou, D. N. , Mol. Phys., 72, 1313 (1991). 
Table 1: Values of the density-independent coefficients of Equations 27, determined using Boublik's equation of state[35] for mixtures. The coefficients are expressed in terms of the species $i$ volume, $b_{i}$, the mole fraction average of the volume, $b=\sum_{i} y_{i} b_{i}$, the shape parameter ratios, $\omega$ and $\psi$, and their derivatives $\omega^{\prime}$ and $\psi^{\prime}$ with respect to $N_{i}$, the number of species $i$ molecules. The shape parameter ratios and their derivatives are defined in Table 2.

$$
\begin{aligned}
& \mathrm{W}\left(y_{a}\right)_{i}^{\left(n_{a}, n_{b}\right)}=-\psi_{i}^{\prime} \\
& \mathrm{X}\left(y_{a}\right)_{i}^{\left(n_{a}, n_{b}\right)}=-\psi_{i}^{\prime}-\omega_{i}^{\prime}-(\omega+1)\left(b_{i} / b\right) \\
& \mathrm{Y}\left(y_{a}\right)_{i}^{\left(n_{a}, n_{b}\right)}=\psi_{i}^{\prime}+2 \omega_{i}^{\prime}-3(\psi-\omega-2)\left(b_{i} / b\right) \\
& \mathrm{Z}\left(y_{a}\right)_{i}^{\left(n_{a}, n_{b}\right)}=(\psi-1)\left(b_{i} / b\right)-\omega_{i}^{\prime}
\end{aligned}
$$

Table 2: The shape parameter ratios, $\omega$ and $\psi$, and their derivatives, $\omega^{\prime}$ and $\psi^{\prime}$ with respect to $N_{i}$, in terms of species shape parameters and mixture shape parameters. The mixture shape parameters $b, s$, and $\bar{r}$ are the mole fraction average $\left(\tau=\sum_{i} y_{i} \tau_{i}\right)$ of the shape parameters for the individual species and $c$ is the mole fraction average of the radius of curvature squared $c=\sum_{i} y_{i} \bar{r}_{i}^{2}$. The species shape parameters for monomers and dimers are defined in Table 3

$$
\begin{aligned}
\omega & =\bar{r} s / b \\
\psi & =\left(c s^{2}\right) /\left(9 b^{2}\right) \\
\omega_{i}^{\prime} & =\partial(N \omega) / \partial N_{i}=\left(r_{i} s+\bar{r} s_{i}-\left(b_{i} / b\right) \bar{r} s\right) / b \\
\psi_{i}^{\prime} & =\partial(N \psi) / \partial N_{i}=\left(r_{i}^{2} s^{2}+2 c s s_{i}-2\left(b_{i} / b\right) c s^{2}\right) /\left(9 b^{2}\right)
\end{aligned}
$$


Table 3: Species shape parameters for monomers and dimers determined from the segment radius, $r_{i}=\sigma_{i} / 2$.

\begin{tabular}{|c||ccc|}
\hline shape & $b_{i}$ & $s_{i}$ & $\bar{r}_{i}$ \\
\hline monomer & $4 \pi r_{i}^{3} / 3$ & $4 \pi r_{i}^{2}$ & $r_{i}$ \\
\hline dimer & $8 \pi r_{i}^{3} / 3$ & $8 \pi r_{i}^{2}$ & $(3 / 2) r_{i}$ \\
\hline
\end{tabular}

Table 4: Coordination number model parameters, where $\sigma_{r}=\sigma_{22} / \sigma_{11}$.

\begin{tabular}{|c||ccc|}
\hline interacting species & $A$ & $B$ & $C$ \\
\hline monomer/monomer & 0.919 & 0.791 & 0.339 \\
\hline dimer/dimer & 1.754 & 4.986 & 1.951 \\
\hline $\operatorname{dim} / \operatorname{mon}\left(\sigma_{r} \geq 1.0\right)$ & $3.207-2.226 \sigma_{r}$ & $-1.347+4.166 \sigma_{r}$ & $-1.795+3.544 \sigma_{r}$ \\
\hline $\operatorname{dim} / \operatorname{mon}\left(\sigma_{r}<1.0\right)$ & $3.010-2.028 \sigma_{r}^{-1}$ & $0.017-2.802 \sigma_{r}^{-1}$ & $-1.997+3.746 \sigma_{r}^{-1}$ \\
\hline
\end{tabular}




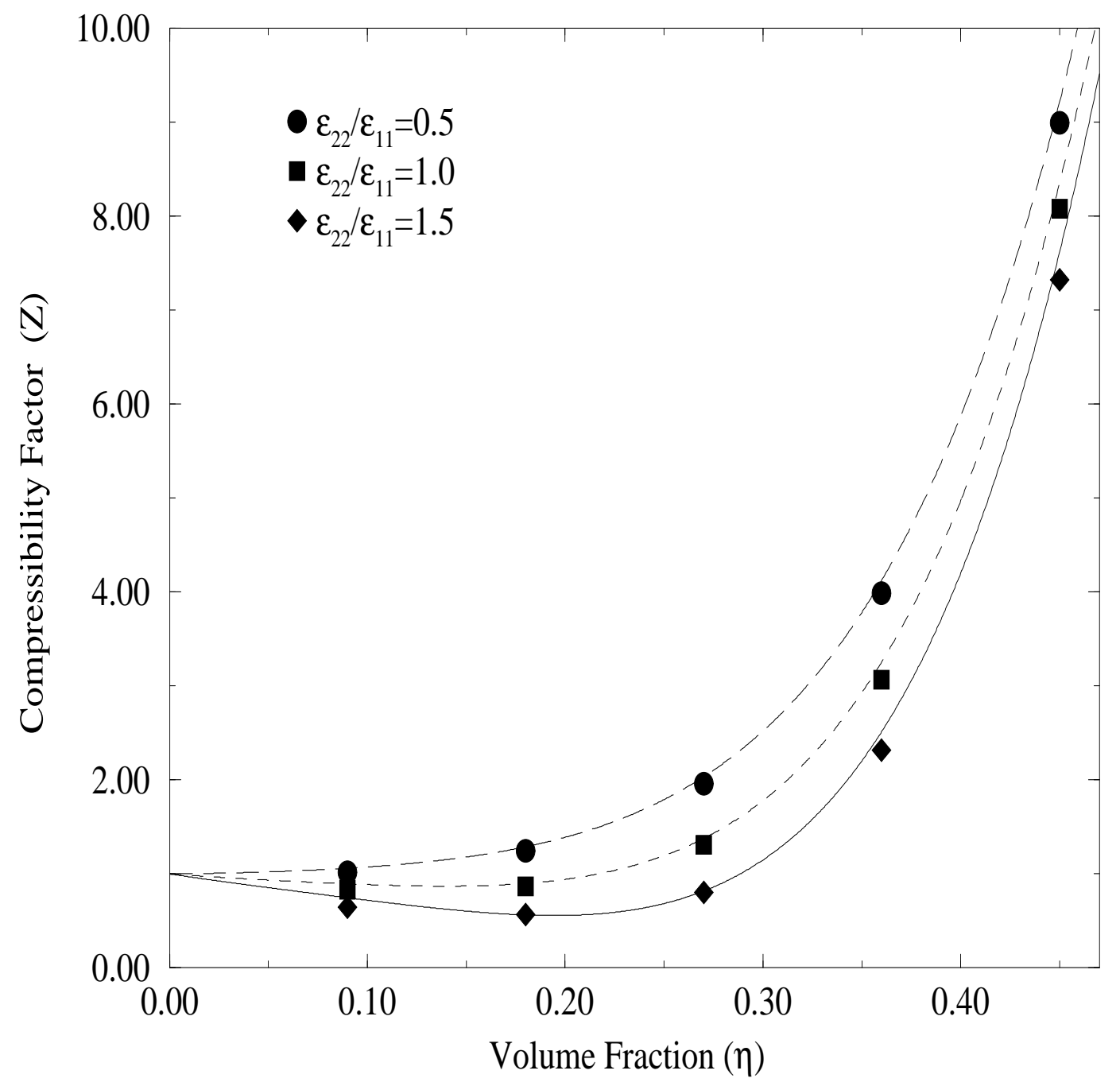

Figure 1: Comparison of GFD equation of state to molecular dynamics simulation results for mixtures of monomers and dimers at $\epsilon_{22} / \epsilon_{11}=0.5,1.0$ and 1.5 , equal segment diameters and equal volume fractions $x_{1} v_{1}=x_{2} v_{2}$ of the two species. 


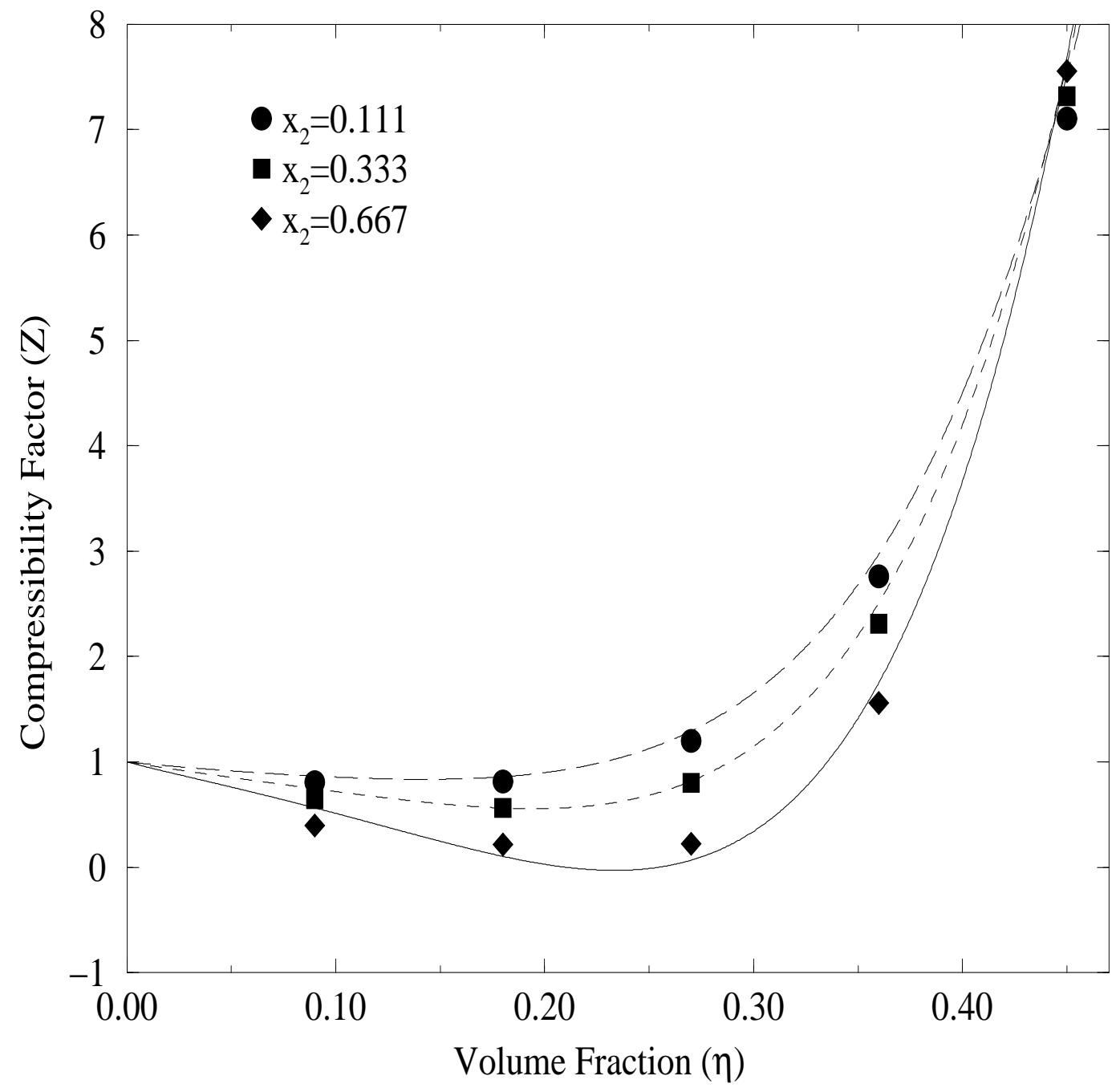

Figure 2: Comparison of GFD equation of state to molecular dynamics simulation results for mixtures of monomers and dimers at $\epsilon_{22} / \epsilon_{11}=1.5$ and $x_{2}=0.111,0.333$, and 0.667 and equal segment diameters. 


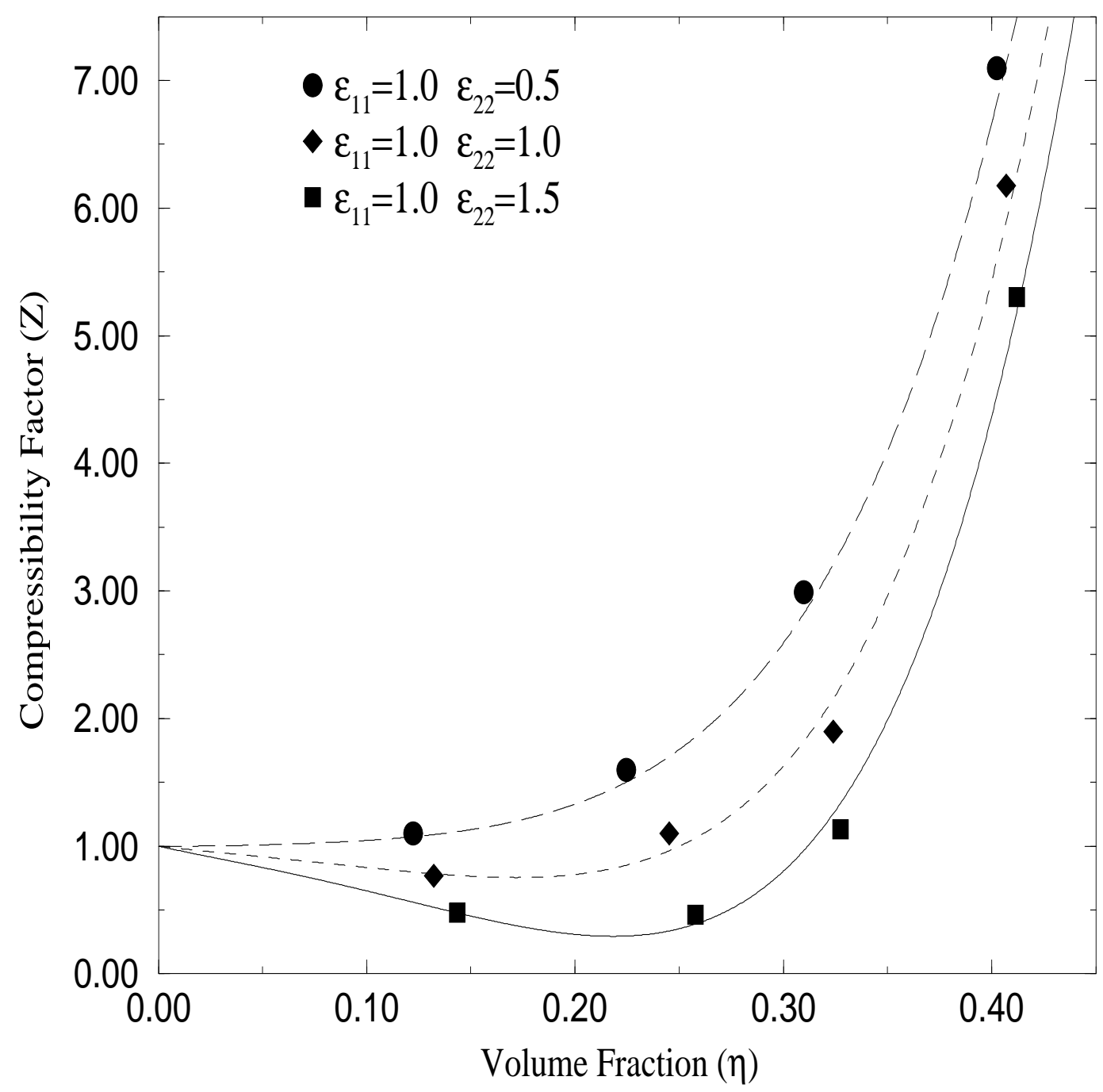

Figure 3: Comparison of GFD equation of state to molecular dynamics simulation results for mixtures of monomers and 8-mers at $\epsilon_{22} / \epsilon_{11}=0.5,1.0$, and 1.5 , equal segment diameters and equal volume fractions $x_{1} v_{1}=x_{2} v_{2}$ of the two species. 

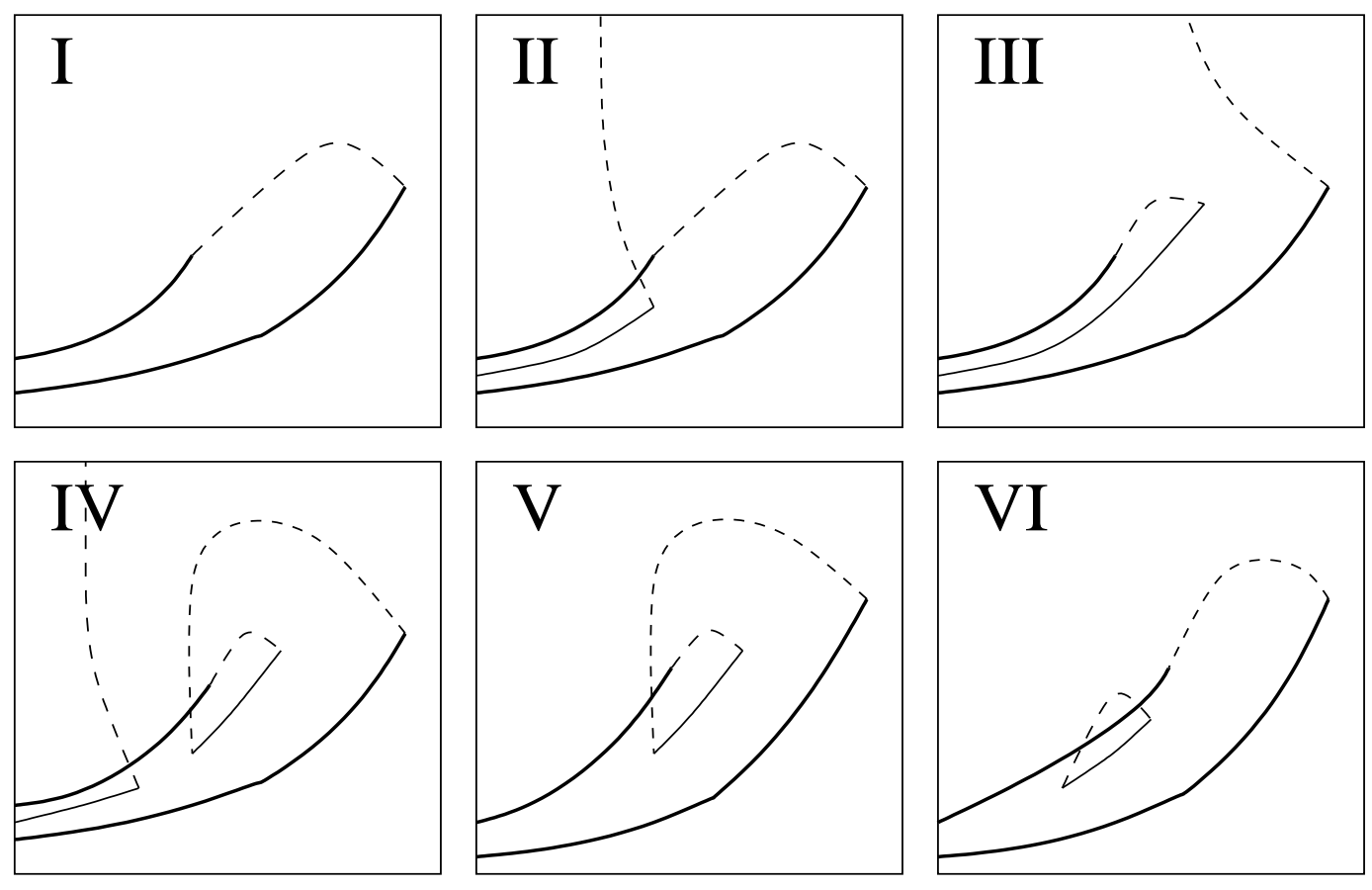

Figure 4: Six classifications of phase behavior. The thicker solid lines are the boiling point of the pure liquids, the thinner solid lines represent three phase coexistence, and the dashed lines are the loci of critical points. 


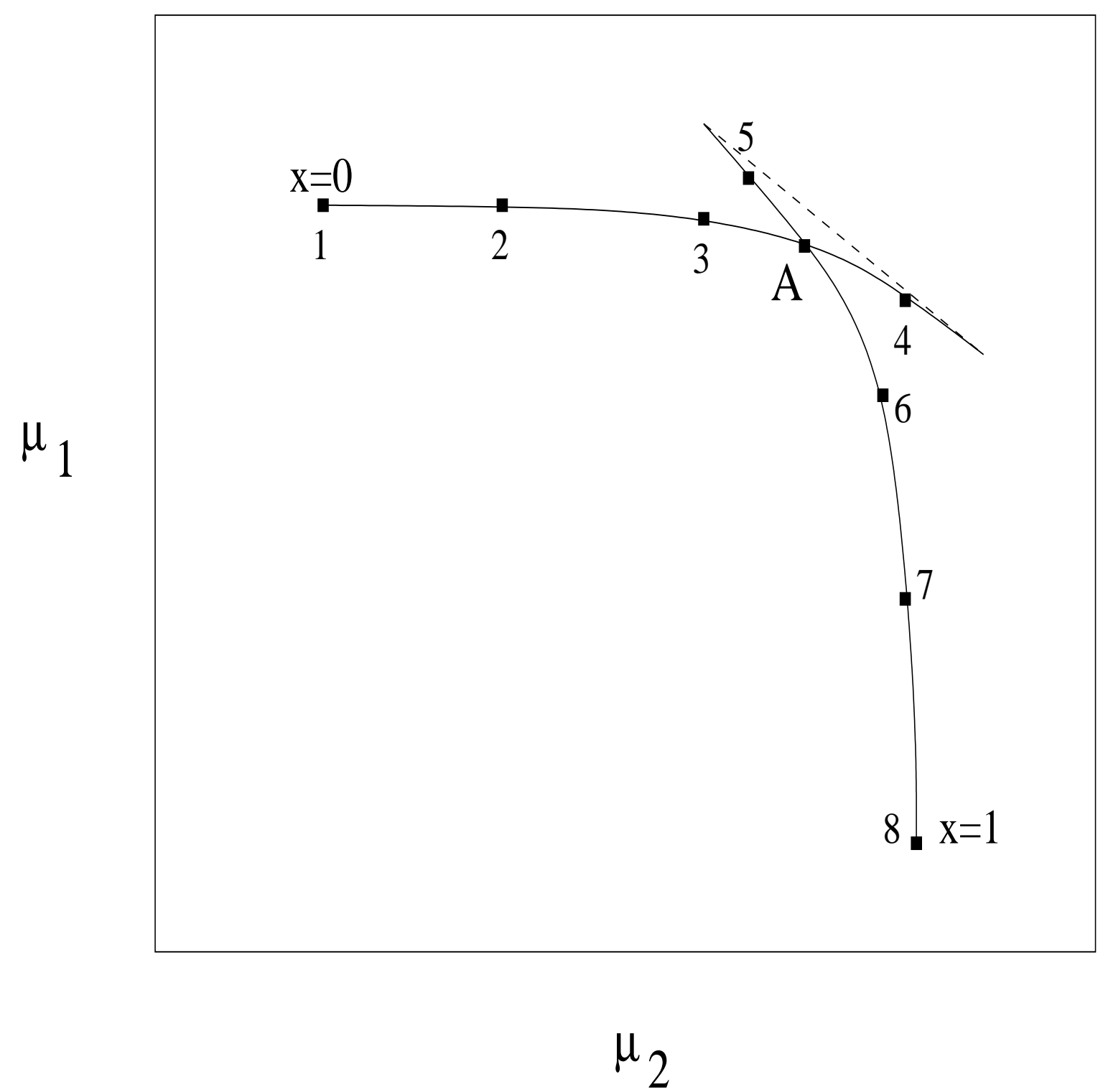

Figure 5: Schematic of a parametrized plot of $\mu_{1}$ vs. $\mu_{2}$ with composition as the parameter. Point A represents the value of $\mu_{1}$ and $\mu_{2}$ at coexistence. 


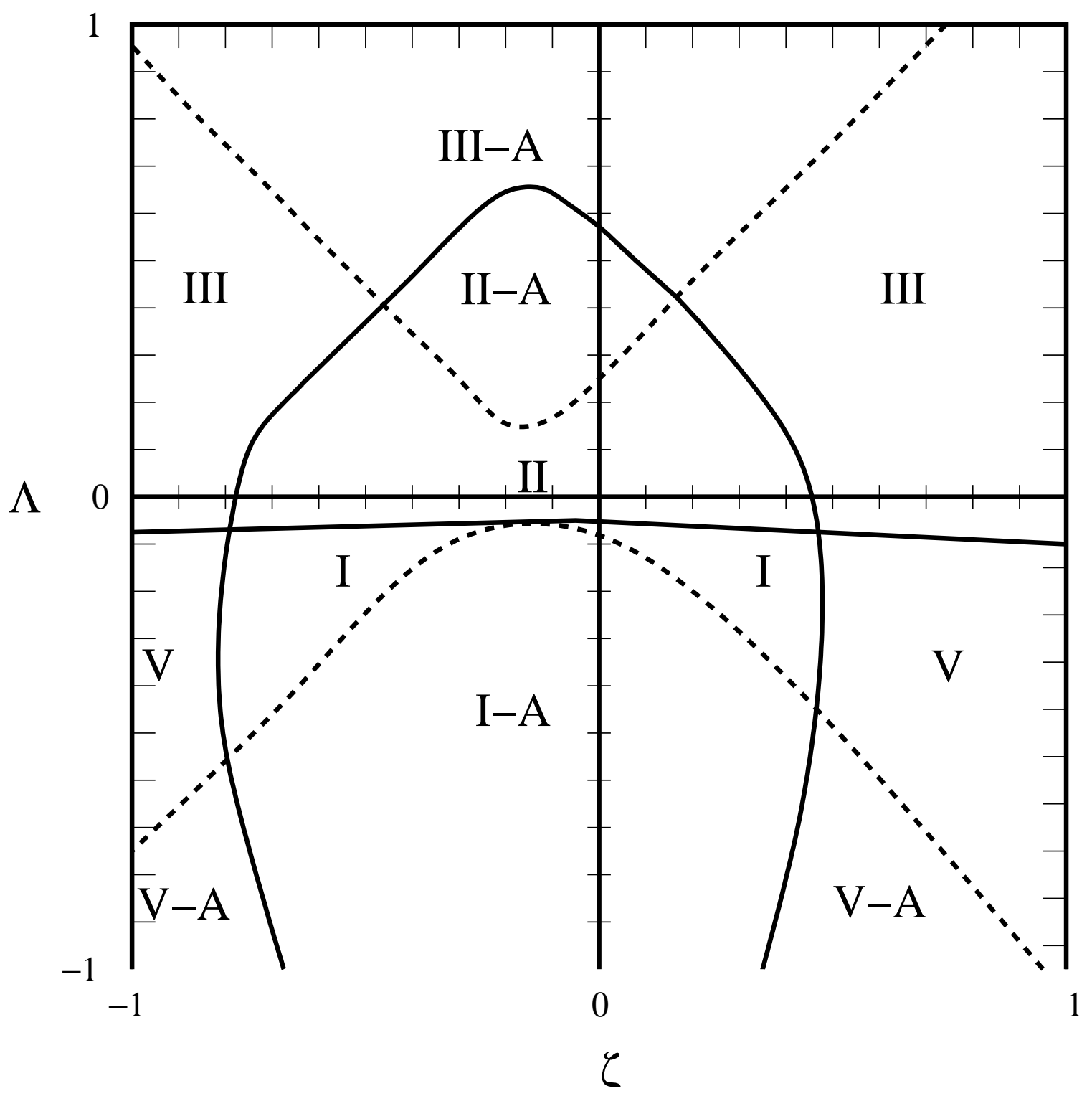

Figure 6: The global phase diagram predicted by the Generalized Flory Dimer theory for a mixture of square-well monomer and dimers. 


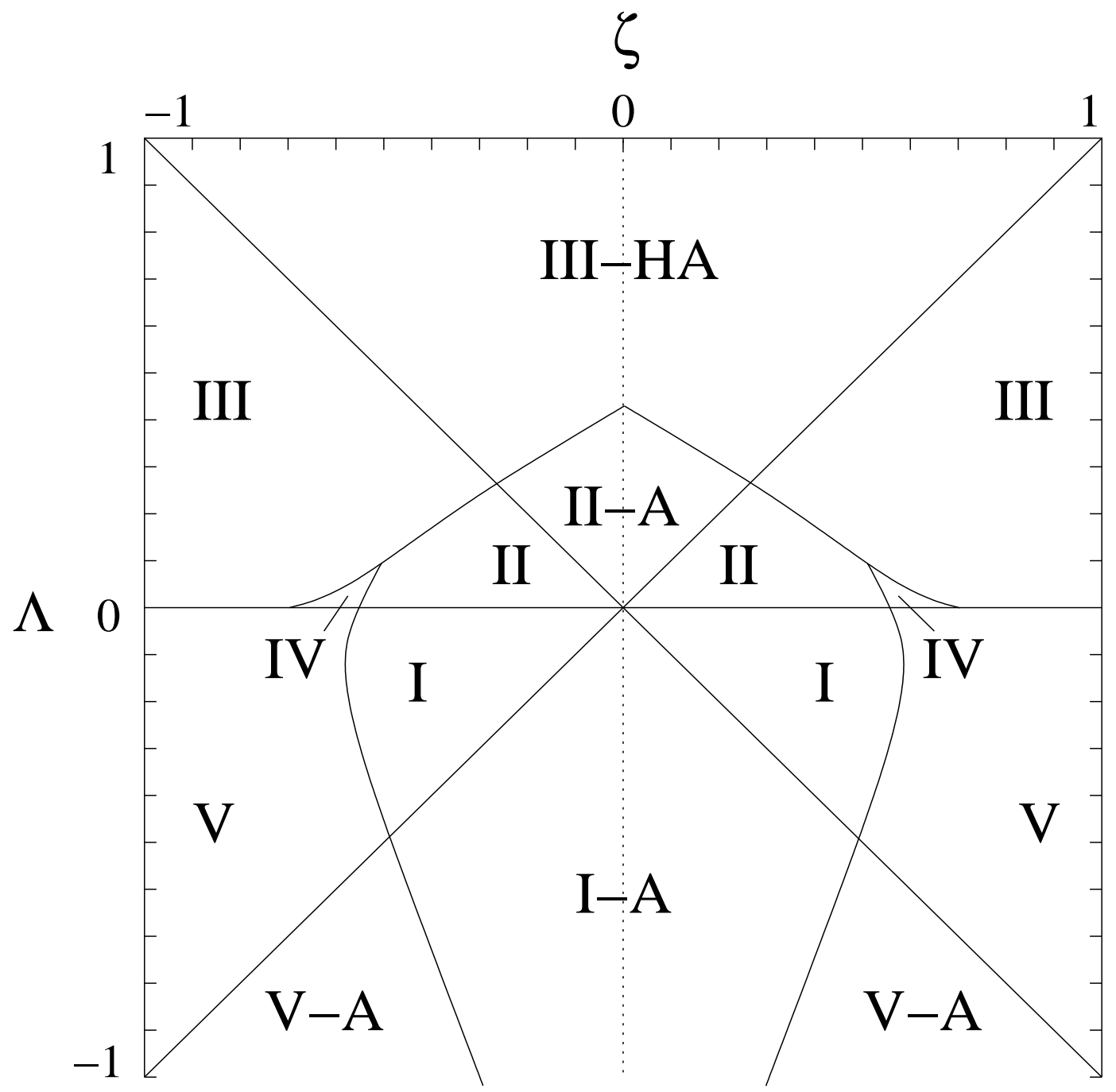

Figure 7: A schematic of the global phase diagram predicted by the van der Waals equation of state for a mixture of equal-sized molecules as calculated by van Konynenburg and Scott [1] 
Appendices 


\section{Appendix A Mixture Excluded Volume}

The Generalized Flory Dimer theory for mixture requires a measure of the average size of the hole required to insert chain molecules with segment diameter $\sigma_{\alpha}$ into a chain fluid mixture of chains of segment diameter $\sigma_{\alpha}$ and $\sigma_{\beta}$. We estimate this hole size to be the average of the volumes that the inserted chain excludes to each type of chain in the mixture.

The volume excluded by a monomer of diameter $\sigma_{\alpha}$ to a monomer of diameter $\sigma_{\beta}$ is given exactly by $\left(\left(v_{e}^{\alpha}(1)\right)_{\beta}=4 \pi / 3\left(\left(\sigma_{\alpha}+\sigma_{\beta}\right) / 2\right)^{3}\right.$. The expression for the volume excluded by a dimer of diameter $\sigma_{\alpha}$ to a monomer of diameter $\sigma_{\beta}$ is given exactly by

$$
\left(\left(v_{e}^{\alpha}(1)\right)_{\beta}=\frac{\pi \sigma_{\alpha}^{3}}{6}\left[2+6\left(\frac{\sigma_{\beta}}{\sigma_{\alpha}}\right)+4.5\left(\frac{\sigma_{\beta}}{\sigma_{\alpha}}\right)^{2}+\left(\frac{\sigma_{\beta}}{\sigma_{\alpha}}\right)^{3}\right]\right.
$$

The volume excluded by chains of three segments or more to monomers are calculated using equations fitted to excluded volume data generated by a program developed by Dodd and Theodorou [36]. The volume excluded by a trimer of segment diameter $\sigma_{\alpha}$ to a monomer of diameter $\sigma_{\beta}$ is given by

$$
\left(\left(v_{e}^{\alpha}(1)\right)_{\beta}=\sigma_{\alpha}^{3}\left[1.57+4.75\left(\frac{\sigma_{\beta}}{\sigma_{\alpha}}\right)+2.99\left(\frac{\sigma_{\beta}}{\sigma_{\alpha}}\right)^{2}+0.52\left(\frac{\sigma_{\beta}}{\sigma_{\alpha}}\right)^{3}\right]\right.
$$

For chains of 4 segments or more, we estimate the volume excluded by a chain of segment diameter $\sigma_{\alpha}$ to a monomer of diameter $\sigma_{\beta}$ using an expression that has the same linear relation as Eq. 10, plus an additional non-linear term

$$
\left(\left(v_{e}^{\alpha}\left(n_{\alpha}\right)\right)_{\beta} \approx\left(v_{e}^{\alpha}(3)\right)_{\beta}+\left(n_{\alpha}-3\right)\left[\left(v_{e}^{\alpha}(3)\right)_{\beta}-\left(v_{e}^{\alpha}(2)\right)_{\beta}\right]+\left(\kappa^{\alpha}\right)_{\beta}\right.
$$


The non-linear term, $\left(\kappa^{\alpha}\right)_{\beta}$, is empirically based and is given by

$$
\left(\kappa^{\alpha}\right)_{\beta}=-\sigma_{\alpha}^{3}\left[0.04915\left(n_{\alpha}-3\right)^{1} .09\left(\frac{\sigma_{\beta}}{\sigma_{\alpha}}\right)^{2} .71\right] .
$$

Given the above equations for the excluded volume, the mixture excluded volume is defined as

$$
\left(Y_{n_{\alpha}}^{\alpha}\right)_{\text {mix }}=\Phi_{\alpha}\left(\frac{v_{e}^{\alpha}\left(n_{\alpha}\right)-v_{e}^{\alpha}(2)}{v_{e}^{\alpha}(2)-v_{e}^{\alpha}(1)}\right)_{\alpha}+\Phi_{\beta}\left(\frac{v_{e}^{\alpha}\left(n_{\alpha}\right)-v_{e}^{\alpha}(2)}{v_{e}^{\alpha}(2)-v_{e}^{\alpha}(1)}\right)_{\beta}
$$

where $\Phi_{i}=\eta_{i} / \eta$ is the occupation fraction of each species. The first term on the right hand side of the equation above is the excluded volume ratio corresponding to the insertion of segments of diameter $\sigma_{\alpha}$ into a pure fluid of segments of diameter $\alpha$. The second term is the excluded volume ratio corresponding to the insertion of segments of diameter $\sigma_{\alpha}$ into a pure fluid of segments of diameter $\beta$. 


\section{Appendix B Code Listing}

In this appendix, we provide the source code for the programs that were used in preparing this thesis. In Section B.1, we list the code used to calculate the locus of critical points. In Section B.2, we list the code used to calculate the phase coexistence envelopes. It should be noted that several subroutines used in Section B.1 are also used in Section B.2, but are listed only in Section B.1. The code was compiled using the Compaq FORTRAN 77 compiler using the -fast and -r8 flags, which enable the use of faster math libraries and convert all REAL type variables to DOUBLE PRECISION variables, respectively. The code was run on our cluster of Alpha processor machines. 


\section{B.1 Code listing for calculating the locus of critical points}

PROGRAM GFDCrit

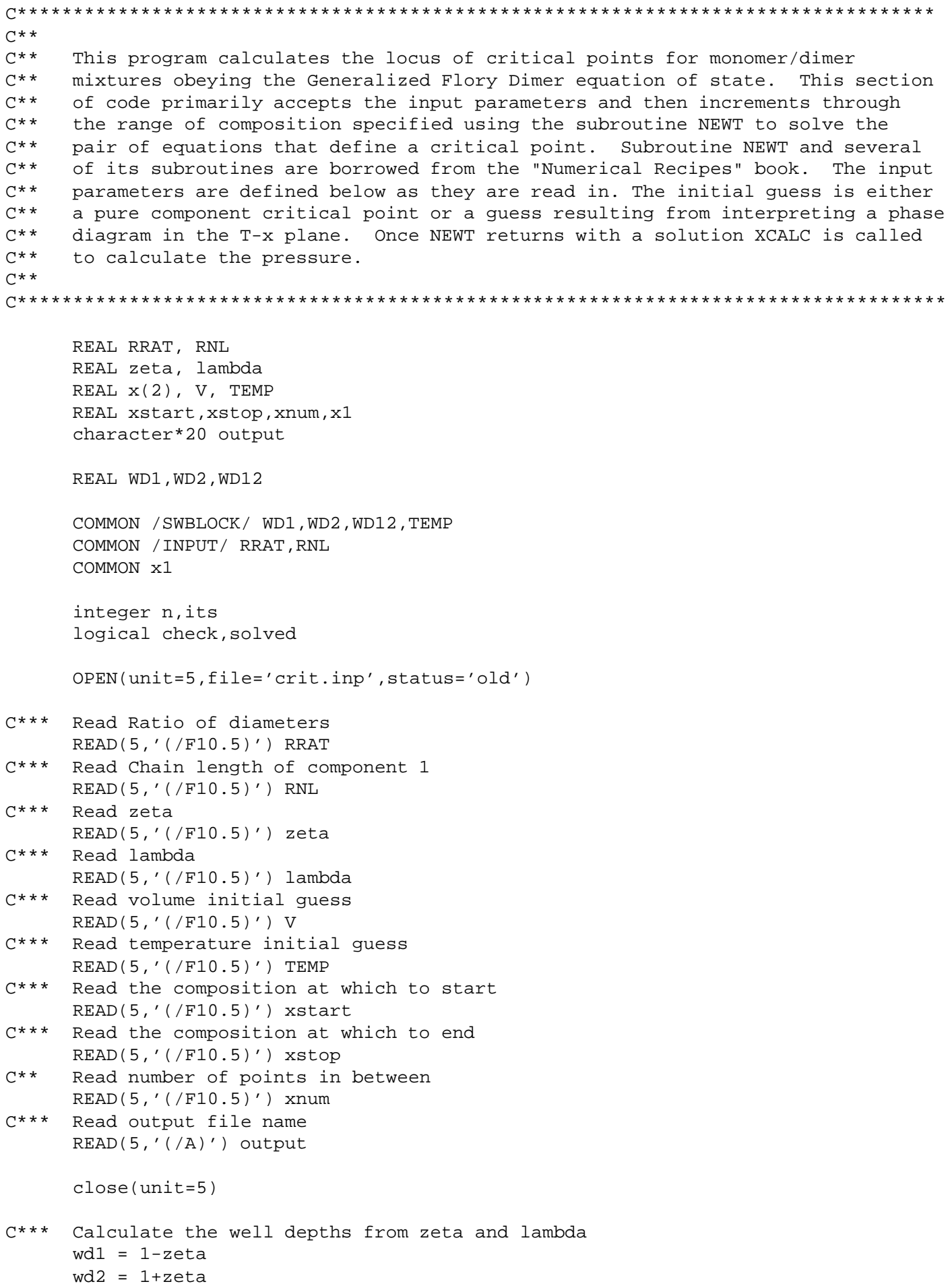


wd12=1- 1 ambda

if (xnum.gt.1) then else

xstep $=($ xstop - xstart $) /($ xnum -1.$)$

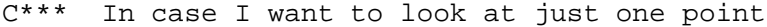
xstep $=(x$ stop $-x$ start $) * 2$.

endif

open (unit=10, file=output, status=' unknown'

do 150 x1=xstart, xstop, xstep

$C * * *$ Call Newton routine to solve the pair of equations for a given composition $\mathrm{x}(1)=\mathrm{V}$

$x(2)=T E M P$

call newt $(x, 2$, check, its, solved, test)

C*** Check to see if it solved within the given number of maximum iterations

$C^{* * *}$ and that it did not converge on a local minimum, then calculate the

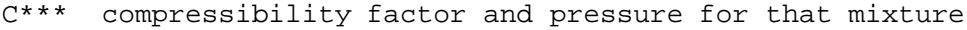

if (.not.check. and.solved) then

call xcalc $(0.0, \mathrm{~V}, \mathrm{x} 1$, Pstar)

write $(10,200)$ TEMP, Pstar, $\mathrm{V}, \mathrm{x} 1$, test, its else

print*, 'not solved', $\mathrm{x} 1$

50 endif

150 continue

200 format (e12.5, 1x, e12.5, 1x, e11.4, 1x, f6.3, 1x, e11.4, 1x, i4)

stop

end

\#include <Fcalc.f >

\#include <funcva.F>

\#include <NEWT.F>

\#include <FDJAC.F>

\#include $<$ FMIN.F>

\#include <LUNLN.F>

\#include <subrtns.f>

\#include <com_vol_dum.f >

\#include <chn_mon_vol.f>

\#include <xcalc.f > 
SUBROUTINE newt ( $\mathrm{x}, \mathrm{n}$, check, its, solved, test)

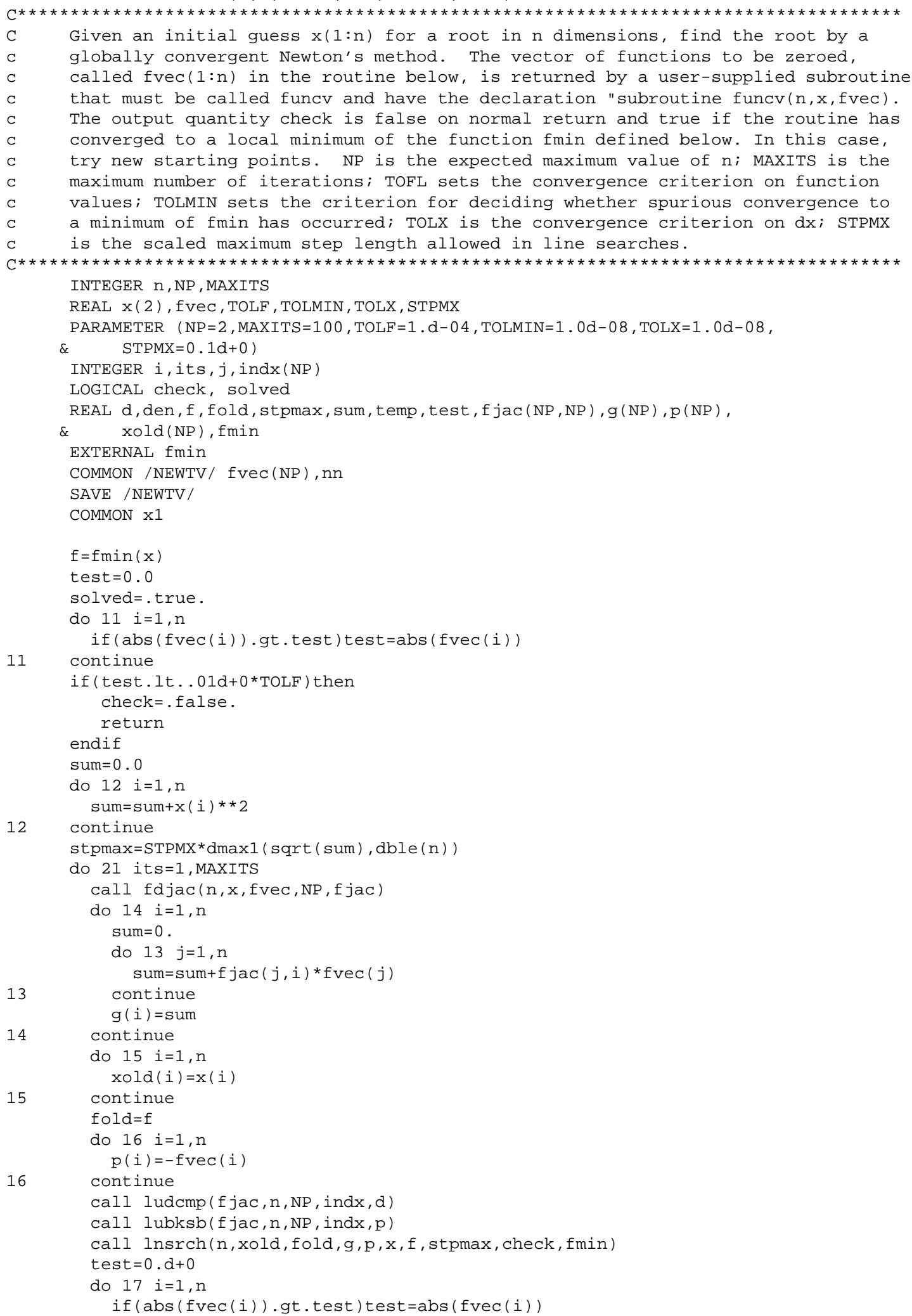


continue

if (test.lt. TOLF) then

check=. false.

return 
DOUBLE PRECISION FUNCTION fmin(x)

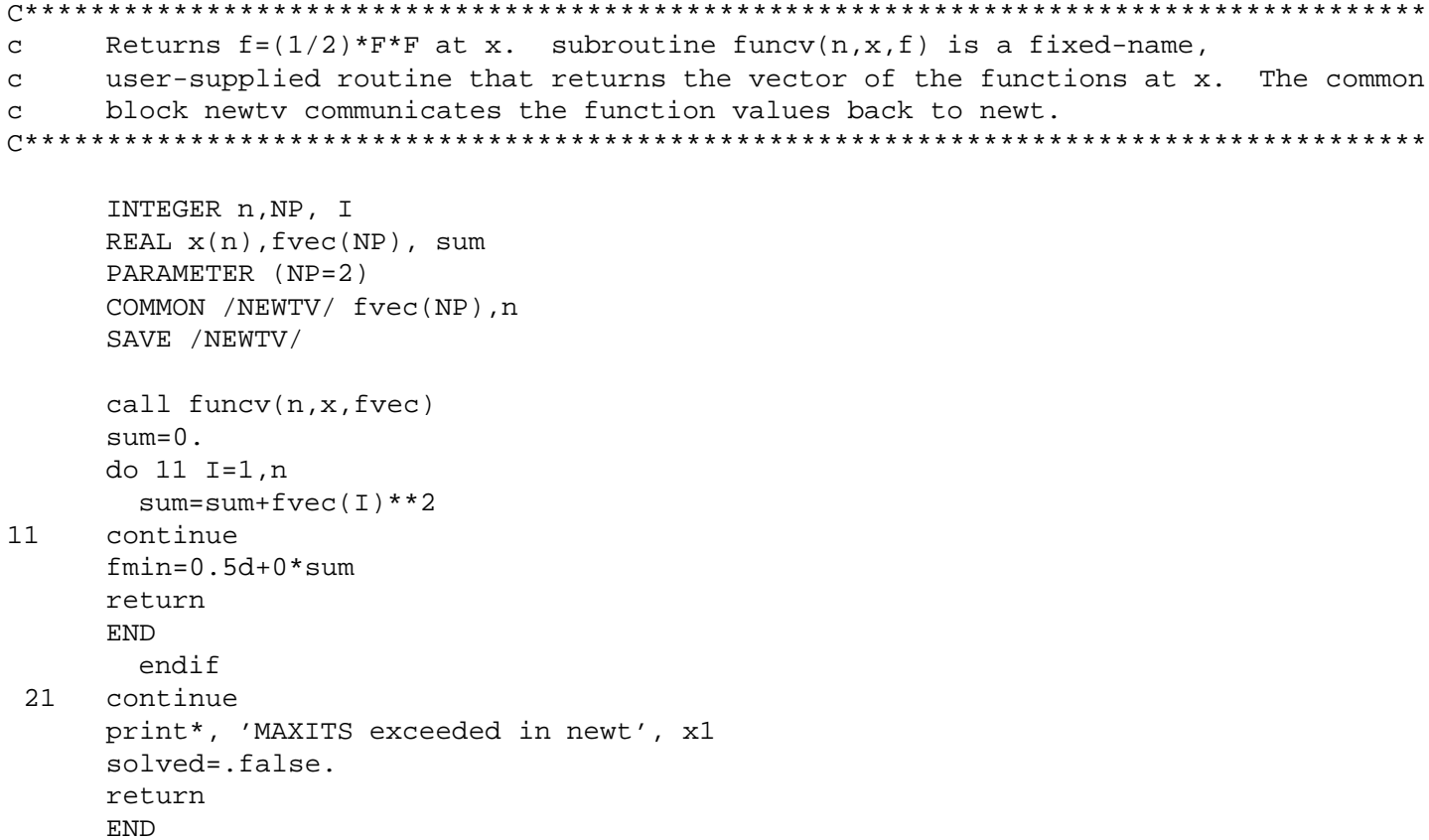


SUBROUTINE funcv $(n, x$, fvec)

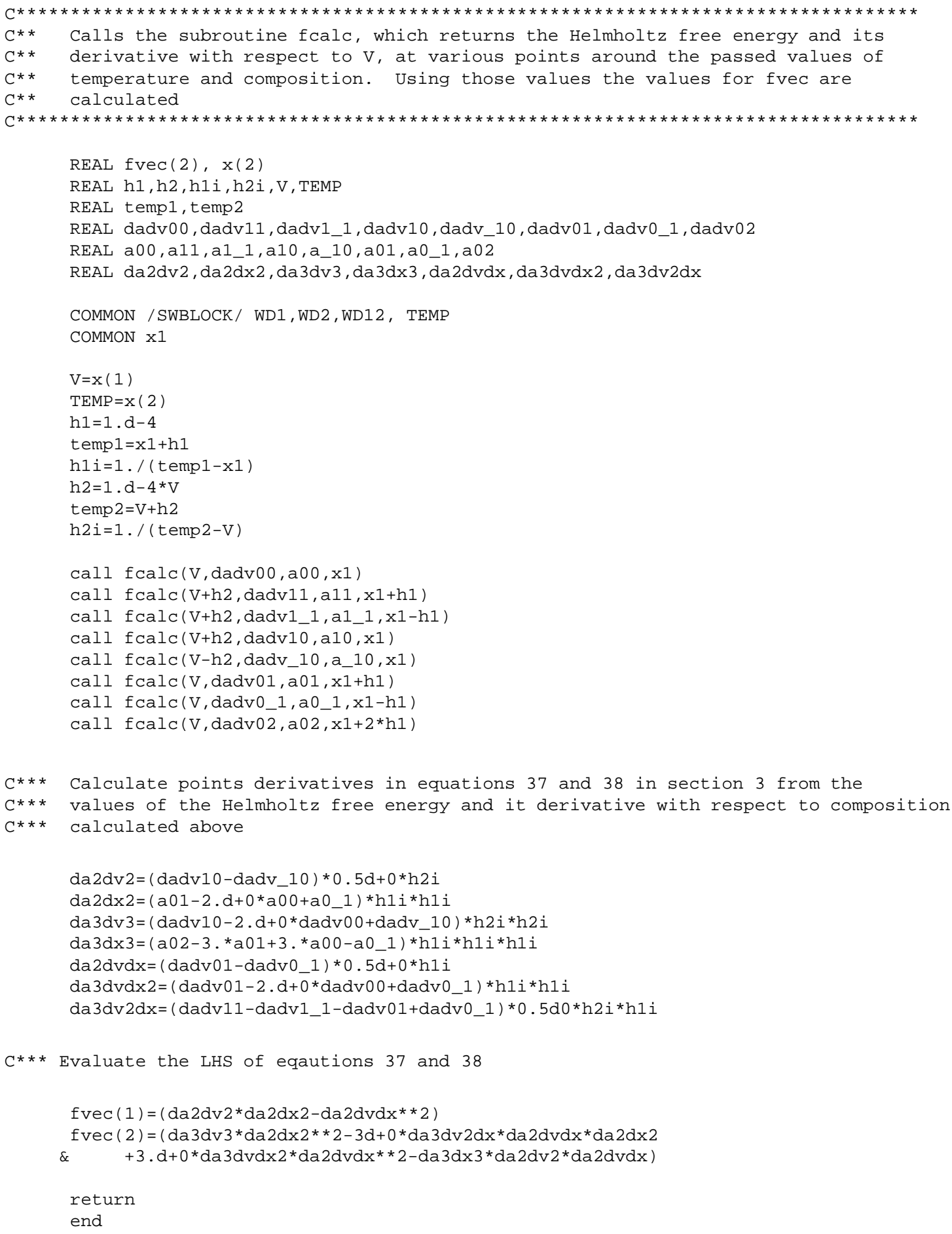




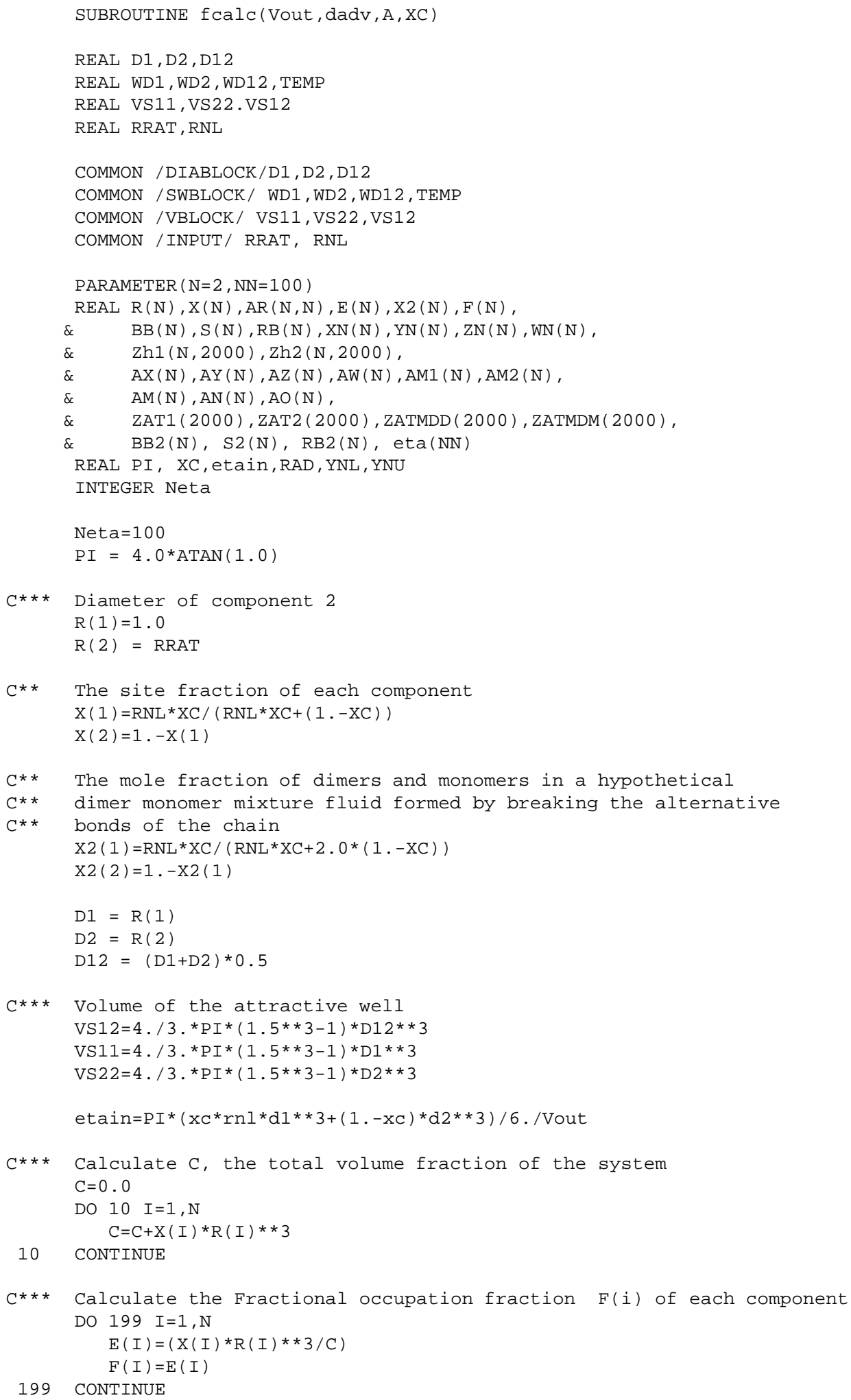




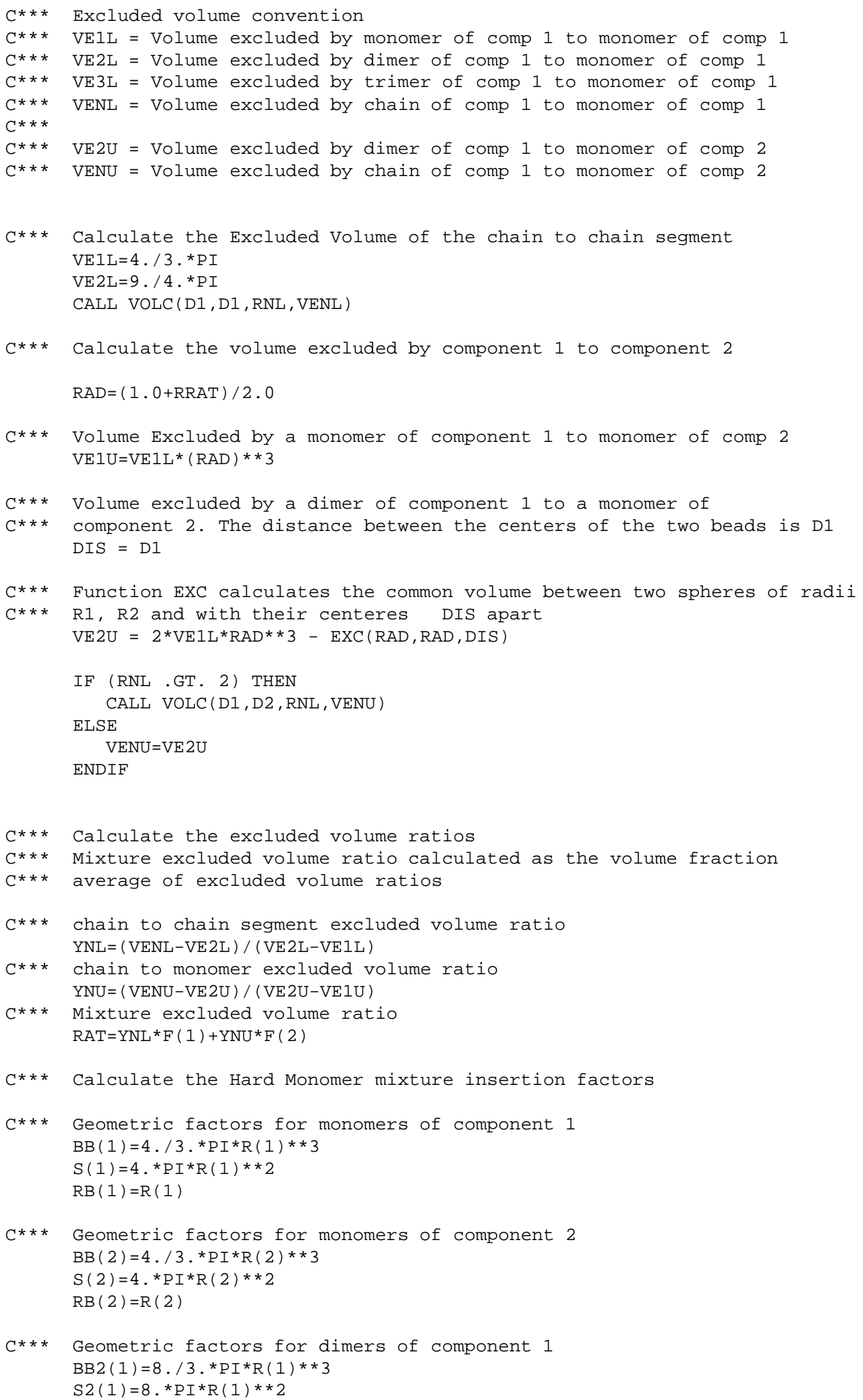




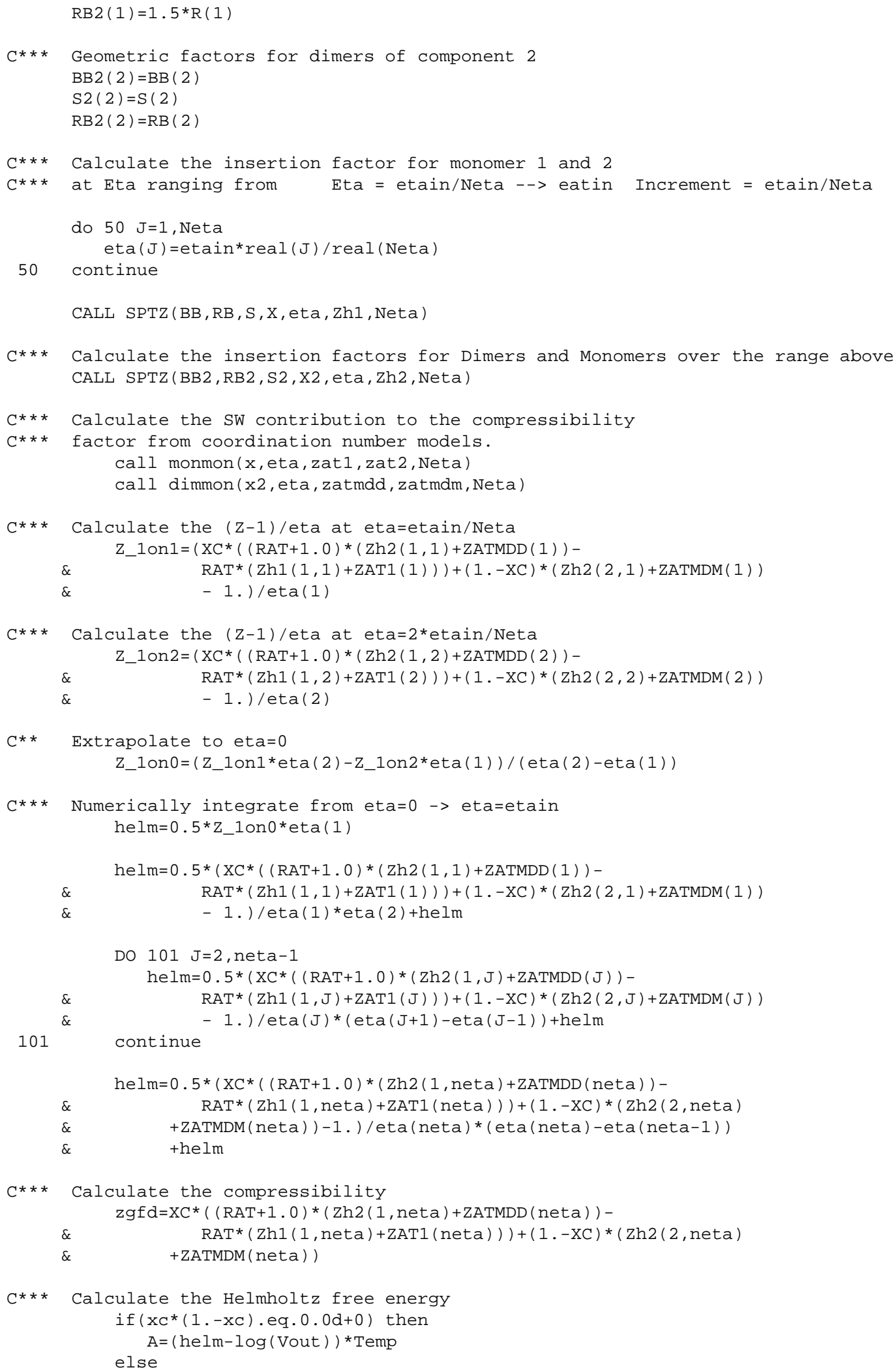


$A=($ helm-log $($ Vout $)+x c * d \log (x c)+(1 .-x c) * d \log ((1 .-x c))){ }^{*} T e m p$ endif

$\mathrm{C}^{* * *}$ The derivative of the Helmholtz free energy with respect to volume

$\mathrm{C} * \star \star$ equals $-\mathrm{P}$ which equals $\mathrm{Z} * \mathrm{~T} / \mathrm{V}$

dadv $=-z g f d /$ Vout $*$ Temp

return

end 
SUBROUTINE VOLC (DIA1, DIA2, CHNLEN, VOL)

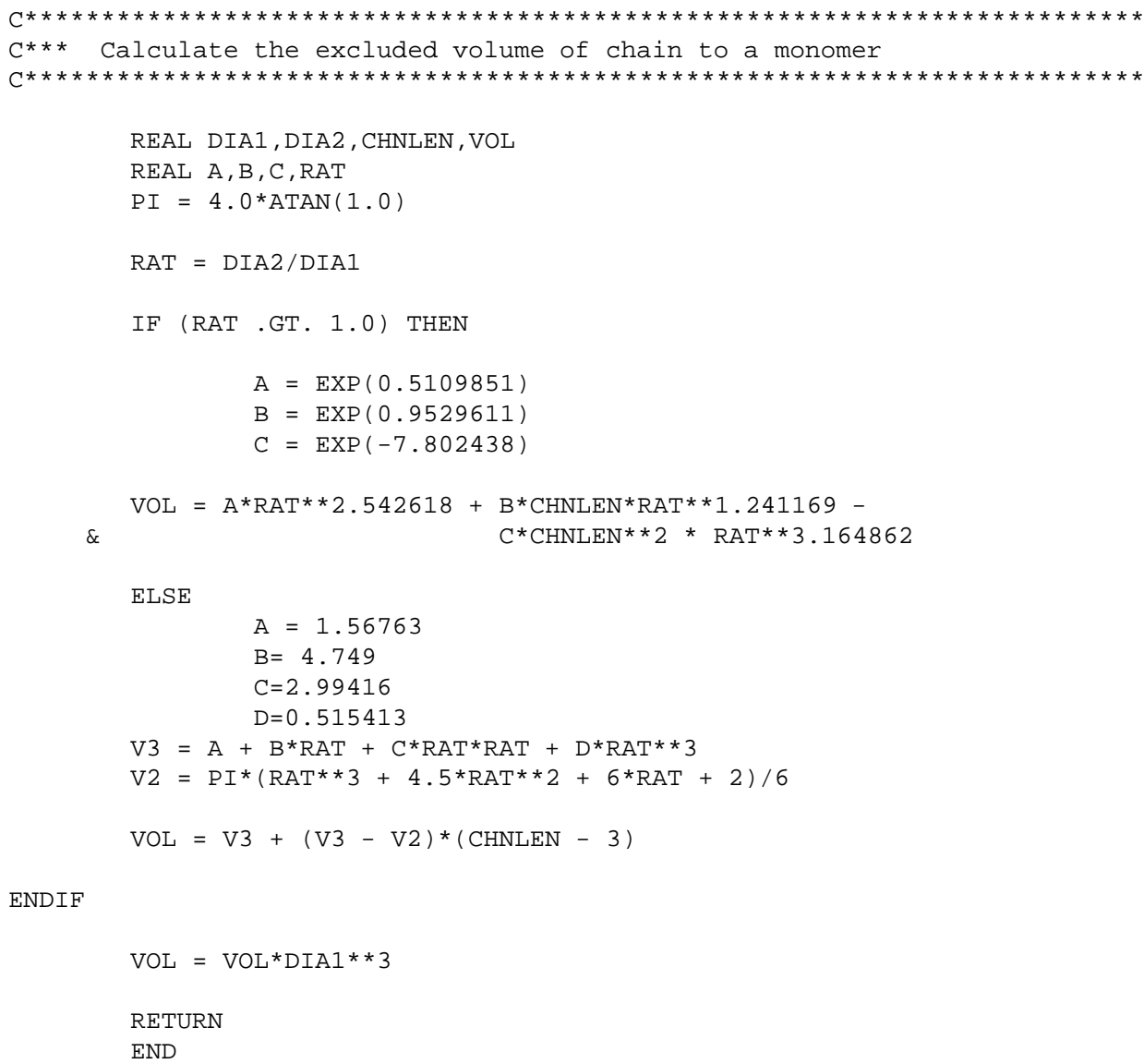


FUNCTION $\quad$ EXC (D1, D2, R)

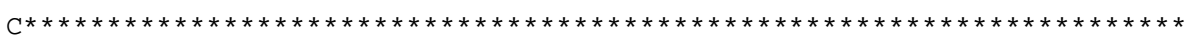

$C^{* *}$ This function calculates the volume common to two

$\mathrm{C}^{* *}$ spheres of radii D1 and D2 whose centers are R apart.

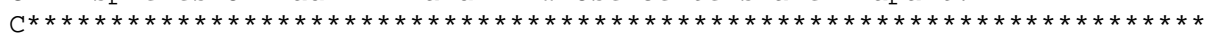

REAL D1, D2, R, PI, EXC, to

$P I=4.0 * \operatorname{ATAN}(1.0)$

to $=2.0 / 3.0 * \mathrm{pi} * \mathrm{~d} 1 * * 3+\mathrm{pi} *(\mathrm{~d} 1 * * 2-\mathrm{d} 2 * * 2$

$\& \quad+r * * 2) *(-10 * d 1 * * 2 * r * * 2+d 1 * * 4-$

\# $2 * \mathrm{~d} 1 * * 2 * \mathrm{~d} 2 * * 2+\mathrm{d} 2 * * 4-2 * \mathrm{~d} 2 * * 2 * r * * 2+r * \star 4)$

$\# / r * * 3 / 24+2.0 / 3.0 * \mathrm{pi} * \mathrm{~d} 2 * * 3-\mathrm{pi}$

$\# *(-r * * 2+\mathrm{d} 1 * * 2-\mathrm{d} 2 * * 2) *(-10 * \mathrm{~d} 2 * * 2 * r * * 2+r * * 4$

\# $-2 * d 1 * * 2 * r * \star 2+d 1 * * 4-2 * d 1 *$

$\# \star 2 * \mathrm{~d} 2 \star \star 2+\mathrm{d} 2 \star \star 4) / r * \star 3 / 24$

$\mathrm{EXC}=\mathrm{to}$

return

end 
DO 100 II $=1$, neta

DO $30 \quad I=1$, NCOMP

$\operatorname{PINS}(I, I I)=(-1.0-W N(I)-Y N(I)-3.0 * \mathrm{ZN}(I)){ }^{\mathrm{ALOG}}(1-\mathrm{ETA}(I I))$

$/ \operatorname{ETA}(\mathrm{II})+((-\mathrm{YN}(\mathrm{I})-3.0 * \mathrm{ZN}(\mathrm{I})-\mathrm{WN}(\mathrm{I}))+\mathrm{ETA}(\mathrm{II})$

$\star(-\mathrm{XN}(\mathrm{I}) / 2.0+5 . / 2 . * \mathrm{YN}(\mathrm{I})+15 . / 2 . * \mathrm{ZN}(\mathrm{I})+$

3. $0 * \mathrm{WN}(\mathrm{I}))+\mathrm{ETA}(\mathrm{II}) * * 2$

* $(-\mathrm{XN}(\mathrm{I}) / 2 .-5 . / 2 . * \mathrm{YN}(\mathrm{I})-11 . / 2 . * \mathrm{ZN}(\mathrm{I})-3 . * \mathrm{WN}(\mathrm{I}))+$

30

CONTINUE

100 CONTINUE

RETURN

END 
SUBROUTINE MONMON (X, eta, Zat1, Zat2, neta)

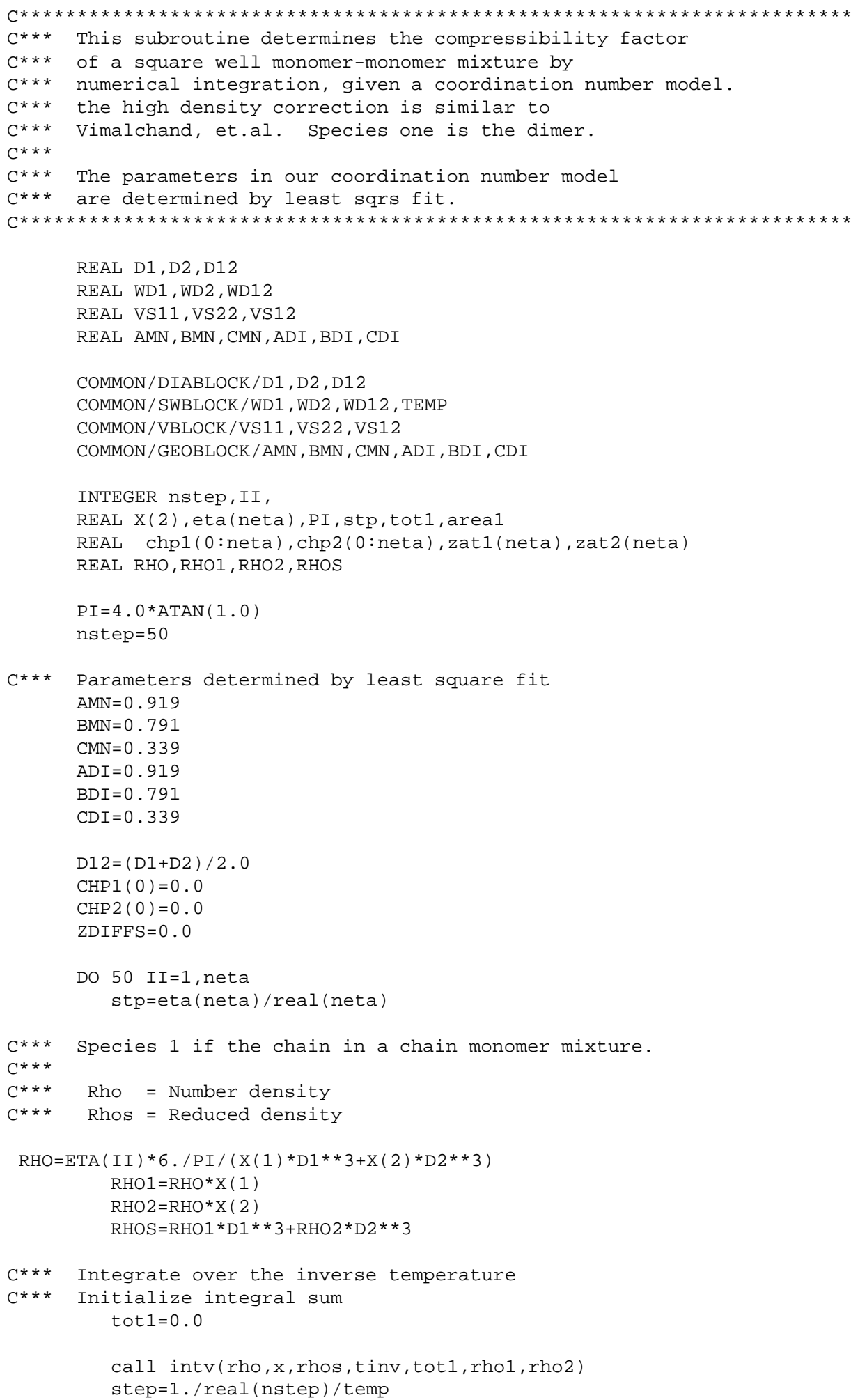




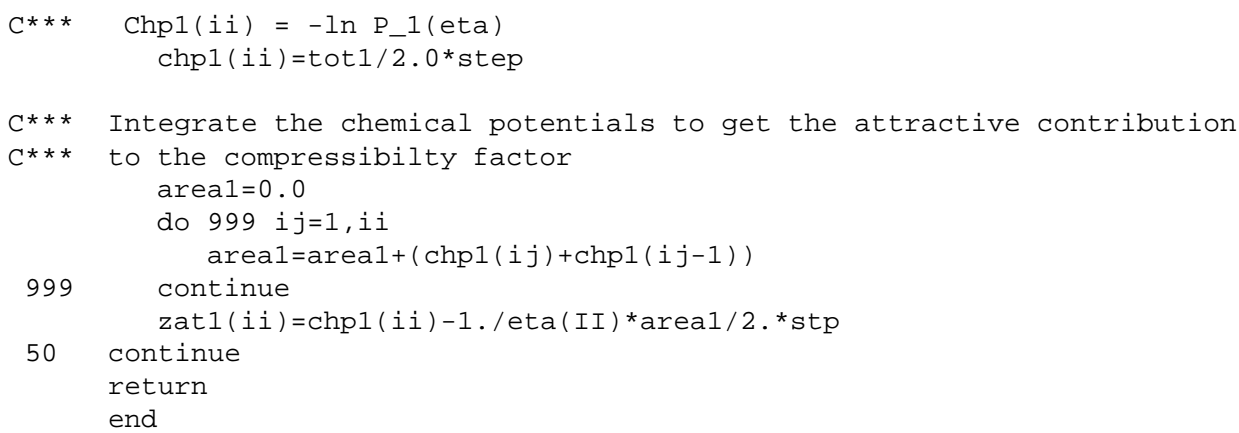


Subroutine Intv(Rho, x, Rhos, Tinv, tot1, Rho1, Rho2)

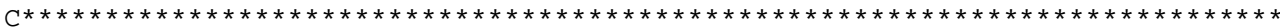

C** This subroutine integrates the expression U-dU/dx with respect to $1 / \mathrm{T}$

$\mathrm{C} \star \star$ from $1 / \mathrm{T}=0 \rightarrow 1 / \mathrm{T}$. This subroutine gets called a lot and profiling the

$C * *$ program revealed that most of the time was being spent here. Hence I

C** did a lot of optimizing by hand and now it is not very reader friendly.

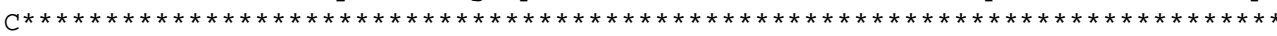

REAL D1, D2, D12

REAL WD1, WD2, WD12

REAL VS11, VS22, VS12

REAL AMN, BMN, CMN, ADI, BDI, CDI

COMMON /DIABLOCK/D1, D2, D12

COMMON/SWBLOCK/WD1, WD2, WD12, TEMP

COMMON/VBLOCK/VS11, VS22, VS12

COMMON/GEOBLOCK/AMN, BMN, CMN , ADI, BDI , CDI

REAL X (2)

REAL Alp, Alpp, zt, ztpd

REAL ZtdRho1, ZtdRho1S, ztpdRho1S, ZtdRho2, ZtdRho2S, ztpdRho2

REAL D13, D123, D23, RhoD13, RhoD123, RhoD23, D1oD2

REAL nstep, $x i$, tinv

REAL Om11, Om12, Om22, Omg, Omgp

REAL Den1, Den12, Den2

REAL Va, Vb, Vc, Va1, Vb1, Vc1

REAL Vi1,Vip1, Vi2

C*** Calculate Alpha and Alpha'

$\mathrm{Alp}=1$.-Rhos/Sqrt (2.)

Alpp $=-1 . /$ Sqrt (2.)

$\mathrm{Zt}=\mathrm{Amn}+\mathrm{Bmn}$ * Rhos $-\mathrm{Cmn} *$ Rhos $*$ * 4

Ztp $=\mathrm{Bmn}-4 .{ }^{\star} \mathrm{Cmn} *$ Rhos $* \star 3$

Ztd $=$ Adi + Bdi * Rhos - Cdi * Rhos * * 4

$\mathrm{Ztpd}=\mathrm{Bdi}-4.0 * \mathrm{Cdi} * \mathrm{Rhos} * \star 3$

ZtdRho1 $=$ Zt d $*$ RHo1

ZtdRho1S $=\mathrm{Ztd}$ *Rho1 *Rho1

ZtpdRho1S $=$ Ztpd*Rho1 * Rho1

ZtdRho2 $=$ Ztd ${ }^{*}$ RHo2

ZtdRho2S=Ztd ${ }^{*}$ Rho2*Rho2

ZtpdRho2S $=$ Ztpd*Rho2 * Rh०2

ZtpdRho2=Ztpd*Rho2

$\mathrm{D} 13=\mathrm{D} 1 * * 3$

D $123=\mathrm{D} 12 * * 3$

D23 $=\mathrm{D} 2 * \star 3$

RhoD 13=Rho*D13

RhoD 123=Rho*D123

RhoD 23=Rho*D23

$\mathrm{D} 1 \mathrm{OD} 2=\mathrm{D} 1 / \mathrm{D} 2$

nstep $=50$

do $10 i=0$, nstep

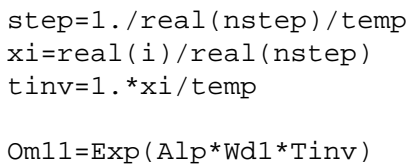




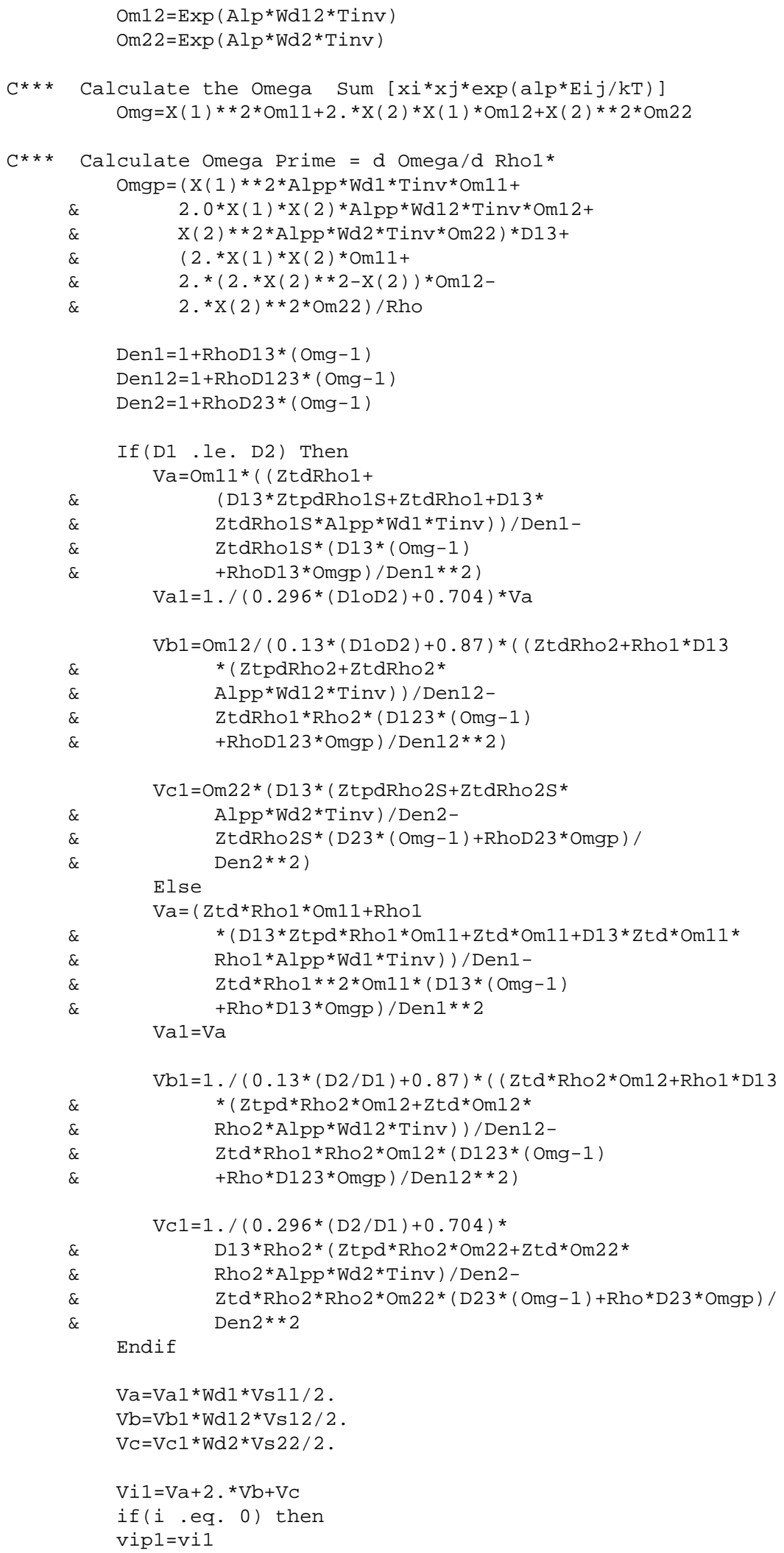


goto 10

endif

$\operatorname{tot} 1=\operatorname{tot} 1-(\operatorname{vi} 1+\mathrm{vip} 1)$

vip1=vi1

10 continue

Vi2 $=0$.

End 
SUBROUTINE DIMMON (X, eta, ZATMDD, ZATMDM, neta)

$C^{*}$ This program determines the compressibility factor

$C^{*}$ of a square well monomer-dimer mixture by numerical integration,

C** given a coordination number model.

$C^{* *}$ the high density correction is similar to

$C^{\star *}$ Vimalchand, et.al.

C** Species one is the dimer.

$C^{\star *}$ the parameters in our coordination number model

C** are determined by least sqrs fit.

REAL D1, D2, D12

REAL WD1, WD2, WD12

REAL VS11, VS22, VS12

REAL AMN, BMN, CMN, ADI, BDI, CDI

REAL ACR, BCR, CCR

COMMON/DIABLOCK/D1, D2, D12

COMMON/SWBLOCK/WD1, WD2, WD12, TEMP

COMMON/GEOBLOCK/AMN, BMN, CMN , ADI, BDI, CDI

COMMON/BLO_LOC/ACR, BCR, CCR

REAL X (2), eta (neta)

REAL CHP1 (0: neta), CHP2 (0: neta)

REAL ZATMDD (neta), ZATMDM (neta)

$\mathrm{PI}=4.0 * \mathrm{ATAN}(1.0)$

NSTEP $=50$

$\mathrm{C} * \star \star A m n, A m n, C m n$ are the parameters for Monomers

C*** Adi, Bdi, Cdi are the parameters for Dimers

C*** Acr, Bcr, Ccr are the parameters for monomer-dimer interaction (Cross interaction)

$\mathrm{AMN}=0.919$

$\mathrm{BMN}=0.791$

$\mathrm{CMN}=0.339$

$\mathrm{ADI}=1.754$

$\mathrm{BDI}=4.986$

$\mathrm{CDI}=1.951$

$\mathrm{RAT}=\mathrm{D} 2 / \mathrm{D} 1$

$\mathrm{D} 12=(\mathrm{D} 1+\mathrm{D} 2) / 2.0$

IF (RAT . GE. 1) THEN

$\mathrm{ACR}=3.207+\mathrm{D} 1 / \mathrm{D} 2 *-2.226$

ELSE

$\mathrm{ACR}=3.010+\mathrm{D} 2 / \mathrm{D} 1 *-2.028$

ENDIF

IF (RAT . GE . 1) THEN

$\mathrm{BCR}=-1.347+\mathrm{D} 1 / \mathrm{D} 2 * 4.166$

ELSE

$\mathrm{BCR}=0.017+\mathrm{D} 2 / \mathrm{D} 1 * 2.802$

ENDIF

IF (RAT . GE. 1) THEN

$\mathrm{CCR}=-1.795+\mathrm{D} 1 / \mathrm{D} 2 * 3.544$

ELSE

$\mathrm{CCR}=-1.997+\mathrm{D} 2 / \mathrm{D} 1 * 3.746$

ENDIF

CHP $1(0)=0.0$

CHP 2 (0) $=0$. 0 
$\mathrm{ZDIFFS}=0.0$

DO 50 II =1, neta

stp=eta (neta)/real (neta)

C*** $\mathrm{X}(1)$ is the molefraction of dimer $\mathrm{RHO}=\mathrm{ETA}(\mathrm{II}) * 6 . / \mathrm{PI} /(2.0 * \mathrm{X}(1) * \mathrm{D} 1 * * 3+\mathrm{X}(2) * \mathrm{D} 2 * \star 3)$

$\mathrm{RHO} 1=\mathrm{RHO} * \mathrm{X}(1)$

$\mathrm{RHO} 2=\mathrm{RHO} * \mathrm{X}(2)$

$\mathrm{RHOS}=2.0 * \mathrm{RHO} 1 * \mathrm{D} 1 * \star 3+\mathrm{RHO} 2 * \mathrm{D} 2 * \star 3$

$C \star * \star$ Integrate over the inverse temperature

$\operatorname{tot} 1=0.0$

$\operatorname{tot} 2=0.0$

call intv2 (rho, $x$, rhos, tinv, tot 1, tot 2, rho1, rho2)

step $=1 . /$ real (nstep) / temp

$\operatorname{chp} 1($ ii) $=\operatorname{tot} 1 / 2.0 *$ step

$\operatorname{chp} 2(i i)=\operatorname{tot} 2 / 2.0 *$ step

$C^{*}$ Integrate the chemical potentials to get the attractive

C** contribution to the compressibilty factor

area $1=0.0$

area $2=0.0$

do $999 i j=1$, $i i$

area1 $=\operatorname{area} 1+(\operatorname{chp} 1(i j)+\operatorname{chp} 1(i j-1))$

area $2=\operatorname{area} 2+(\operatorname{chp} 2(i j)+\operatorname{chp} 2(i j-1))$

999 continue

zatmdd $($ ii) $=\operatorname{chp} 1$ (ii) $-1 . /$ eta $($ II $) *$ area $1 / 2 . *$ stp

zatmdm $($ ii $)=\operatorname{chp} 2$ (ii) -1 . / eta (II) *area $2 / 2$. stp

$z a t t=z a t m d d(i i) * X(1)+z a t m d m(i i) * X(2)$

50 continue

return

end 
SUBROUTINE INTV2 (RHO, X, RHOS, TINV, tot1, tot2, RHO1, RHO2)

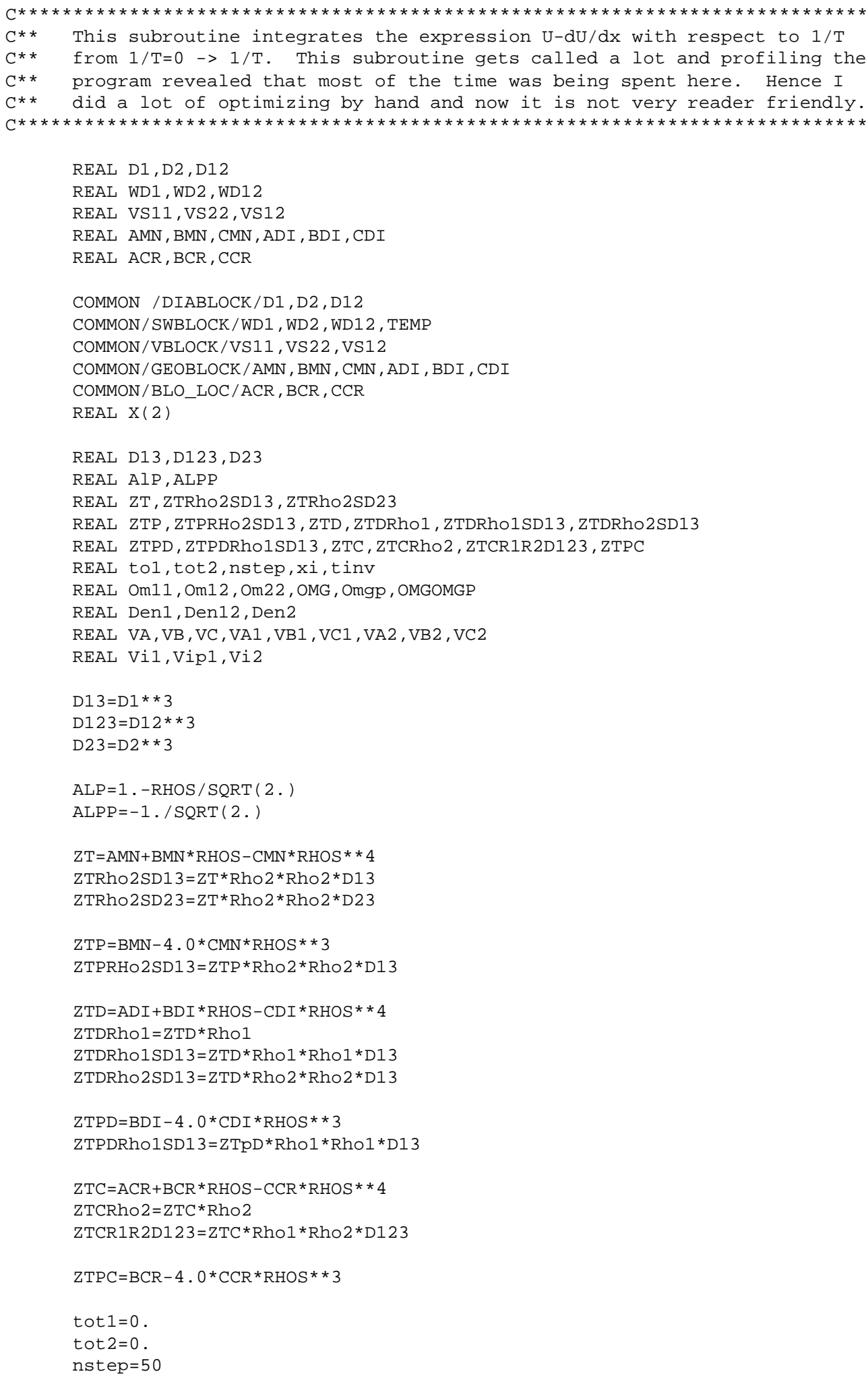


do $10 i=0$, nstep

step $=1$. /real (nstep) /temp

$x i=\operatorname{real}(i) /$ real (nstep)

tinv=1./temp*xi

C*** Calculate Omega

Om11 $=\operatorname{Exp}(\mathrm{Alp}$ *Wd1 *Tinv $)$

Om12 $=\operatorname{Exp}(\mathrm{Alp} * \mathrm{Wd} 12 * \mathrm{Tinv})$

Om22 $=\operatorname{Exp}(\mathrm{Al} p * W d 2 * \mathrm{Tinv})$

$\mathrm{OMG}=\mathrm{X}(1) \star \star 2 * \mathrm{OM} 11+2 . * \mathrm{X}(2) * \mathrm{X}(1) \star \mathrm{OM} 12+\mathrm{X}(2) \star \star 2 * \mathrm{OM} 22$

$\mathrm{DEN} 1=1+\mathrm{RHO} * \mathrm{D} 13 *(\mathrm{OMG}-1)$

DEN12 $=1+$ RHO $*$ D $123 *(O M G-1)$

$\mathrm{DEN} 2=1+\mathrm{RHO} * \mathrm{D} 23 *(\mathrm{OMG}-1)$

$\mathrm{C} \star \star \star$ Calculate Omega Prime

$\mathrm{OMGP}=(\mathrm{X}(1) * \star 2 * \mathrm{ALPP} * \mathrm{WD} 1 * \mathrm{TINV} * \mathrm{OM} 11+$

$\& \quad 2.0 * \mathrm{X}(1) * \mathrm{X}(2) * \mathrm{ALPP} * \mathrm{WD} 12 * \mathrm{TINV} * \mathrm{OM} 12+$

$\mathrm{X}(2) * \star 2 * \mathrm{ALPP} * \mathrm{WD} 2 * \mathrm{TINV} * \mathrm{OM} 22) * \mathrm{D} 13+$

$(2 . * \mathrm{X}(1) * \mathrm{OM} 11+2 . *(2 . * \mathrm{X}(2)-1). * \mathrm{OM} 12-$

$2 . * X(2) * \mathrm{OM} 22) * \mathrm{X}(2) / \mathrm{RHO}$

OMGOMGP $=($ OMG -1$)+$ Rho $*$ OMGP

C*** d Rhos/d Rho1 $=$ D13

C*** d Rho/ d Rho1 = 1

$\mathrm{VA} 1=(2.0 * \mathrm{ZTDRHO} 1 * \mathrm{OM} 11+(\mathrm{ZTPDRHO} 1 \mathrm{SD} 13$

\& $\quad$ *OM11+ZTDRho1SD13*OM11*ALPP *WD1*TINV) ) /DEN1

\& $\quad$ ZTDRHO1SD13*OM11*OMGOMGP / DEN 1 * *2

$\mathrm{VB} 1=(\mathrm{ZTCRHO} 2 * \mathrm{OM} 12+\mathrm{RHO} 1 * \mathrm{RHO} 2 * \mathrm{D} 13 *(\mathrm{ZTPC} * \mathrm{OM} 12$

$\& \quad+\mathrm{ZTC} * \mathrm{OM} 12 * \mathrm{ALPP} * \mathrm{WD} 12 * \mathrm{TINV})) / \mathrm{DEN} 12-$

\& $\quad \mathrm{ZTCR} 1 \mathrm{R} 2 \mathrm{D} 123 * \mathrm{OM} 12$ *OMGOMGP $/ \mathrm{DEN} 12 * * 2$

$\mathrm{VC} 1=(\mathrm{ZTPRH} \circ 2 \mathrm{SD} 13 * \mathrm{OM} 22+\mathrm{ZTRh} 02 \mathrm{SD} 13 * \mathrm{OM} 22$ *

\& $\quad$ ALPP *WD2*TINV) /DEN2-

\& $\quad \mathrm{ZTRHO} 2 \mathrm{SD} 23$ * OM22 * OMGOMGP / DEN 2 * * 2

$\mathrm{VA}=\mathrm{VA} 1 * \mathrm{WD} 1 * \mathrm{VS} 11 / 2$.

$\mathrm{VB}=\mathrm{VB} 1 * \mathrm{WD} 12 * \mathrm{VS} 12 / 2$

$\mathrm{VC}=\mathrm{VC} 1 * \mathrm{WD} 2 * \mathrm{VS} 22 / 2$.

$\mathrm{Vi} 1=\mathrm{VA}+2 \cdot * \mathrm{VB}+\mathrm{VC}$

$\mathrm{OMGP}=(\mathrm{X}(2) * \star 2 * \mathrm{ALPP} * \mathrm{WD} 2 * \mathrm{TINV} * \mathrm{Om} 22+$

$\& \quad 2 . * \mathrm{X}(1) * \mathrm{X}(2) * \mathrm{ALPP} * \mathrm{WD} 12 * \mathrm{TINV} * \mathrm{Om} 12+$

$\left.\mathrm{X}(1) * \star 2 * \mathrm{ALPP} * \mathrm{WD} 1{ }^{*} \mathrm{TINV} * \mathrm{Om} 11\right) * \mathrm{D} 23+$

$(2 . * \mathrm{X}(2) \star \mathrm{X}(1) \star \mathrm{O} m 22+$

$2 . *(2 . * \mathrm{X}(1) * \star 2-\mathrm{X}(1)) * \mathrm{Om} 12-$

$2 . \star X(1) \star \star 2 *$ Om11)/RHO

$\mathrm{VA} 2=(2.0 * \mathrm{ZT} * \mathrm{RHO} 2 * \mathrm{OM} 22+\mathrm{RHO} 2 * \star 2 * \mathrm{D} 23 *(\mathrm{ZTP} * \mathrm{OM} 22$

$\& \quad+\mathrm{ZT} * \mathrm{OM} 22 * \mathrm{ALPP} * \mathrm{WD} 2 * \mathrm{TINV})) / \mathrm{DEN} 2-$

$\mathrm{ZT} * \mathrm{RHO} 2 * \star 2 * \mathrm{OM} 22 *((\mathrm{OMG}-1) \star \mathrm{D} 23$

$+\mathrm{RHO} * \mathrm{D} 23 * \mathrm{OMGP}) / \mathrm{DEN} 2 * * 2$

$\mathrm{VB} 2=(\mathrm{ZTC} * \mathrm{RHO} 1 * \mathrm{OM} 12+\mathrm{RHO} 2 * \mathrm{RHO} 1 * \mathrm{D} 23 *(\mathrm{ZTPC} * \mathrm{OM} 12+$

$\& \quad \mathrm{ZTC} * \mathrm{OM} 12 * \mathrm{ALPP} * \mathrm{WD} 12 * \mathrm{TINV})) / \mathrm{DEN} 12-$

\& $\quad \mathrm{ZTC} * \mathrm{RHO} 1 * \mathrm{RHO} 2 * \mathrm{OM} 12 *(\mathrm{D} 123 *(\mathrm{OMG}-1)$

$\& \quad+\mathrm{RHO} * \mathrm{D} 123 * \mathrm{OMGP}) / \mathrm{DEN} 12 * \star 2$

$\mathrm{VC} 2=\mathrm{D} 23 * \mathrm{RHO} 1 * * 2$ * $(\mathrm{ZTPD} * \mathrm{OM} 11+\mathrm{ZTD} * \mathrm{OM} 11$ *

ALPP *WD 1 * TINV) / DEN $1-$

$\mathrm{ZTD}$ * $\mathrm{RHO} 1$ * $\mathrm{RHO} 1$ *OM11* (D13* (OMG-1) +RHO *D13*OMGP $) /$

DEN $1 * * 2$ 
$\mathrm{VA}=\mathrm{VA} 2 * \mathrm{WD} 2 * \mathrm{VS} 22 / 2$.

$\mathrm{VB}=\mathrm{VB} 2 * \mathrm{WD} 12 * \mathrm{VS} 12 / 2$.

$\mathrm{VC}=\mathrm{VC} 2 * \mathrm{WD} 1 * \mathrm{VS} 11 / 2$.

$\mathrm{Vi} 2=\mathrm{VA}+2 \cdot{ }^{*} \mathrm{VB}+\mathrm{VC}$

if $(i$.eq. 0$)$ then

vip1=vi1

vip2=vi2

goto 10

endif

$C^{* *}$ tot is the total attractive interaction for

C** the square well fluid.

tot $1=\operatorname{tot} 1-(\operatorname{vi} 1+\mathrm{vip} 1)$

tot $2=\operatorname{tot} 2-(v i 2+v i p 2)$

vip1=vi1

vip2=vi2

10 continue

RETURN

END 
SUBROUTINE ludcmp $(a, n, n p$, indx, d)

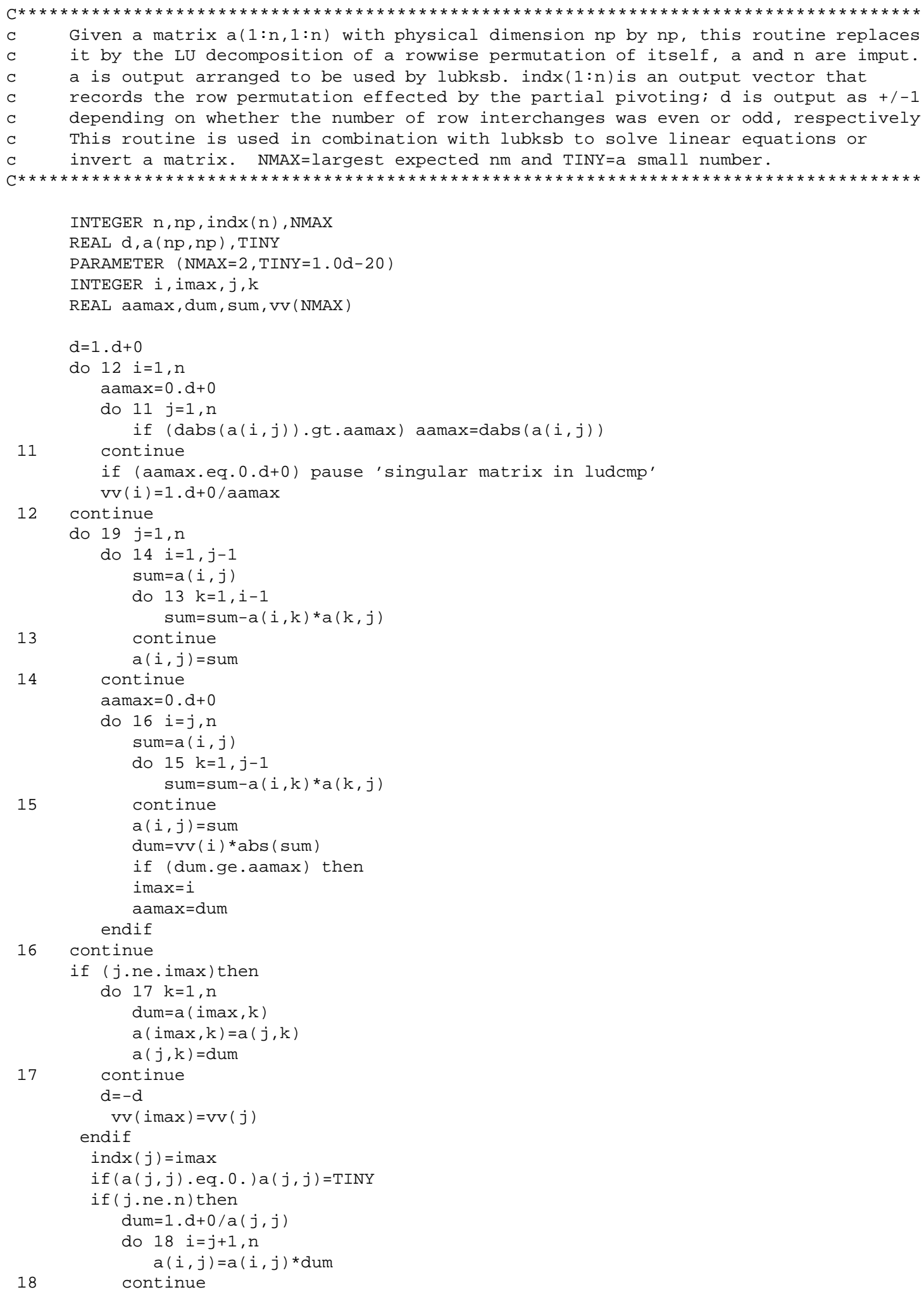


endif

19 continue

return

END 
$\operatorname{SUBROUTINE}$ lubksb $(\mathrm{a}, \mathrm{n}, \mathrm{np}, \mathrm{indx}, \mathrm{b})$

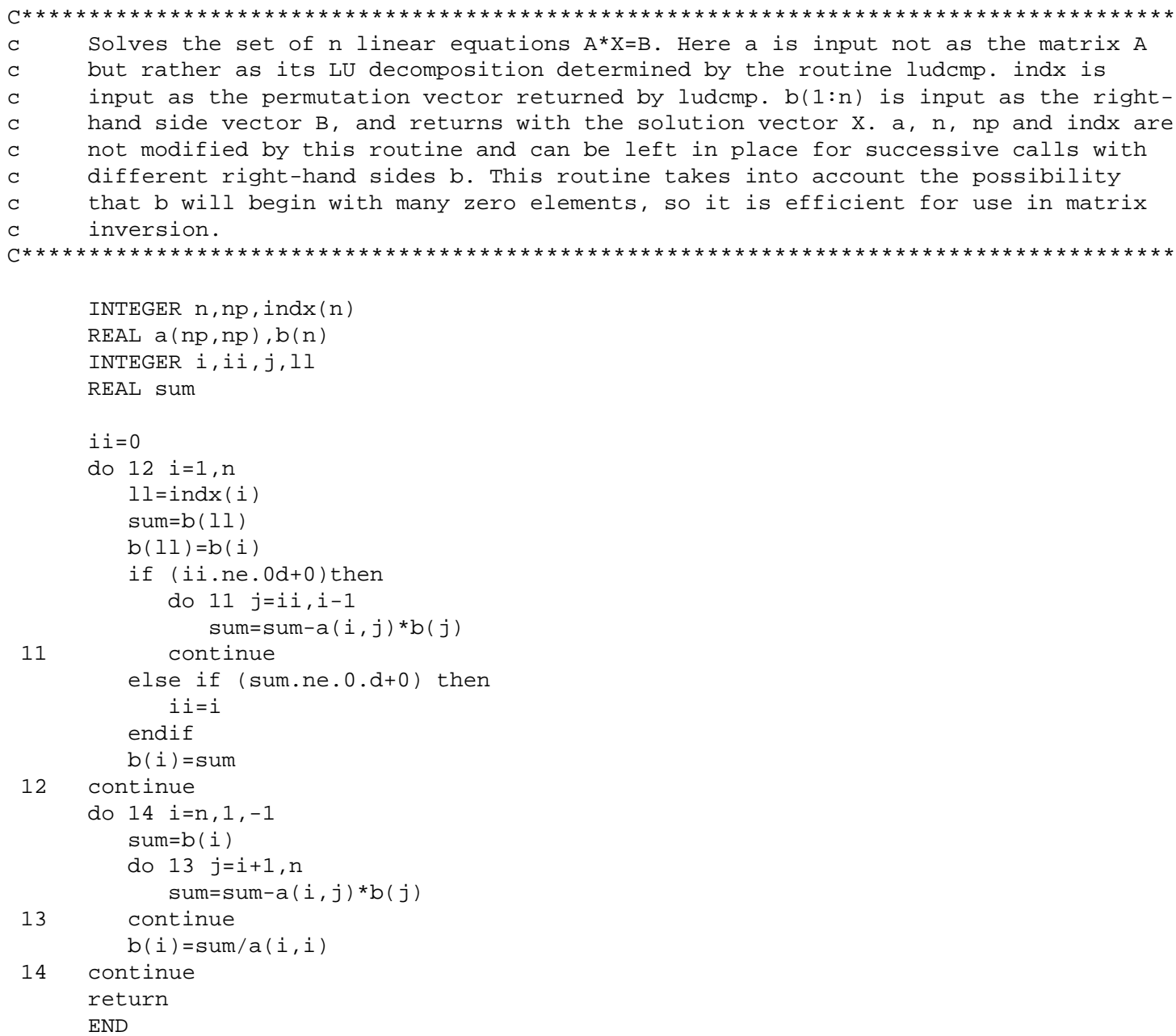


SUBROUTINE lnsrch ( $\mathrm{n}, \mathrm{xold}$, fold, $\mathrm{g}, \mathrm{p}, \mathrm{x}$, f, stpmax, check, func)

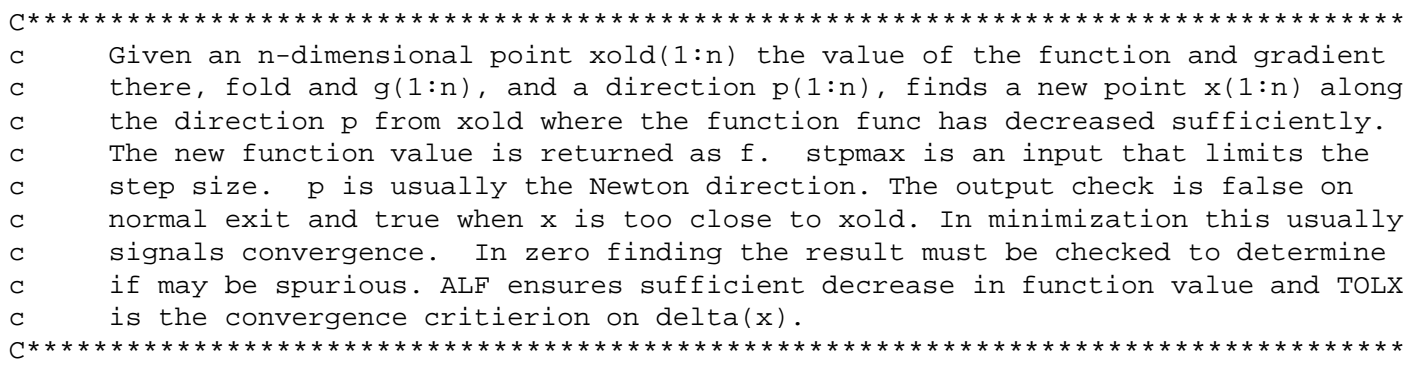

REAL D1, D2, D12

REAL RRAT, RNL, $x 1$

COMMON /DIABLOCK/ D1, D2, D12

COMMON /INPUT/ RRAT, RNL

COMMON $\times 1$

INTEGER $\mathrm{n}$, i, its

LOGICAL check

REAL f, fold, stpmax, $g(n), p(n), x(n), x o l d(n)$, func, ALF, TOLX, d1, d2

Real a, alam, alam2, alamin,b, disc, f2, fold2, rhs1, rhs2, slope,

\& sum, temp, test, tmplam

PARAMETER (ALF $=1 . d-4$, TOLX $=1 . d-7)$

EXTERNAL func

check $=$.false.

$P I=4.0 d+0 * d a T A N(1.0 d+0)$

its $=0$

sum $=0 . d+0$

do $11 i=1, n$

sum $=s u m+p(i) * p(i)$

11 continue

sum=dsqrt (sum)

if (sum.gt.stpmax) then

do $12 i=1, n$

$p(i)=p(i) *$ stpmax / sum

12 continue

endif

slope $=0 . d+0$

do $13 i=1, n$

slope $=$ slope+g (i) *p (i)

13 continue

test $=0$.

do $14 i=1, n$

temp $=$ dabs $(p(i)) / \operatorname{dmax} 1(\operatorname{dabs}(\operatorname{xold}(i)), 1 . d+0)$

if (temp.gt.test) test $=$ temp

14 continue

alamin $=$ TOLX/test

a l $a m=1 . d+0$

1 continue

do $15 i=1, n$

$x(i)=x o l d(i)+a l a m * p(i)$

15 continue

etain $=P I *(x 1 * R N L * D 1 * * 3+(1-x 1) * D 2 * * 3) / 6 d+0 / x(1)$

if (etain.gt.0.70d+0) then

print*, 'eta>0.7'

alam $=0.5 * a l a m$

goto 1

elseif(etain.lt.0.0) then

print*, 'eta<1' 


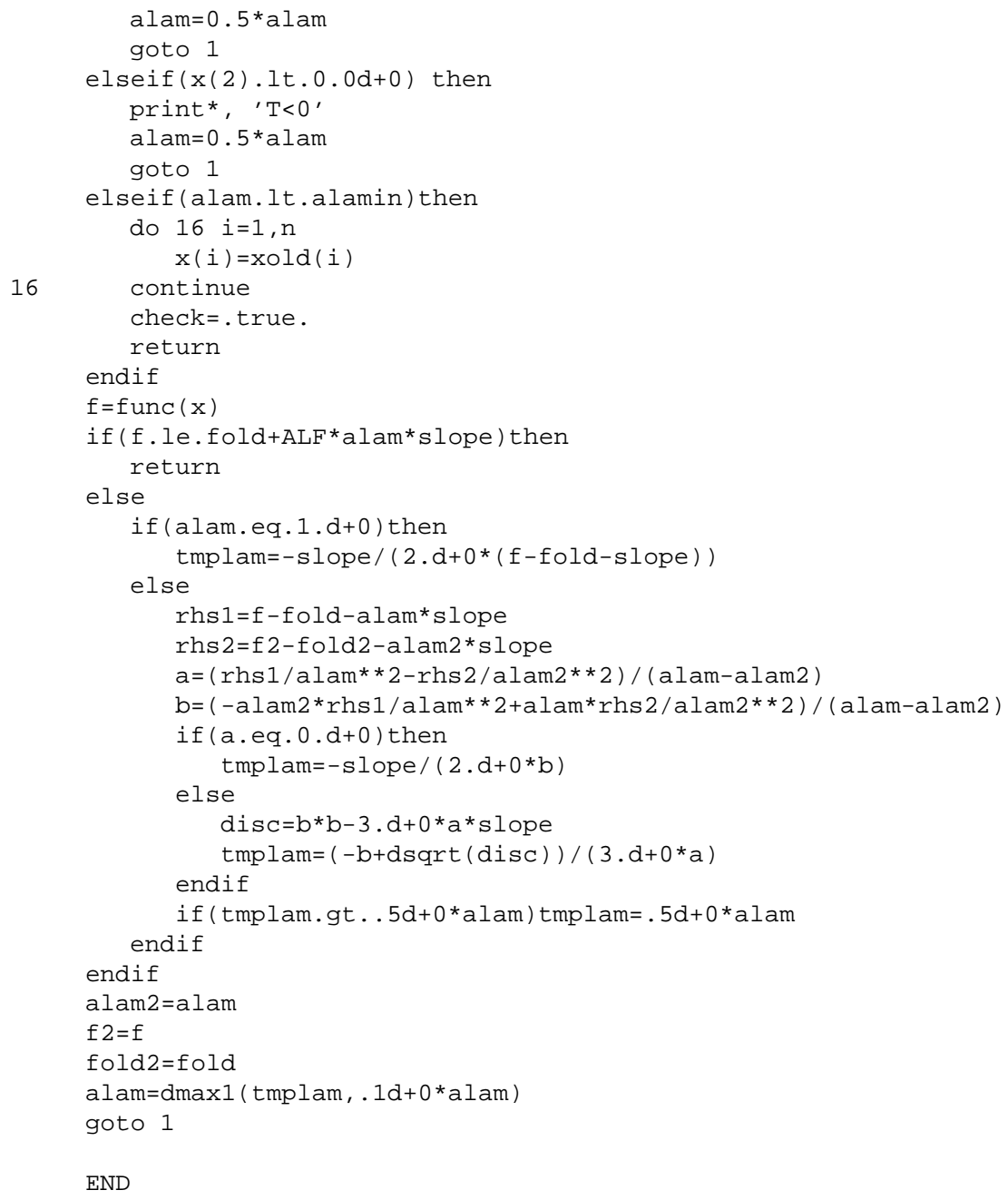

END 


\section{B.2 Code listing for calculating coexistence points}

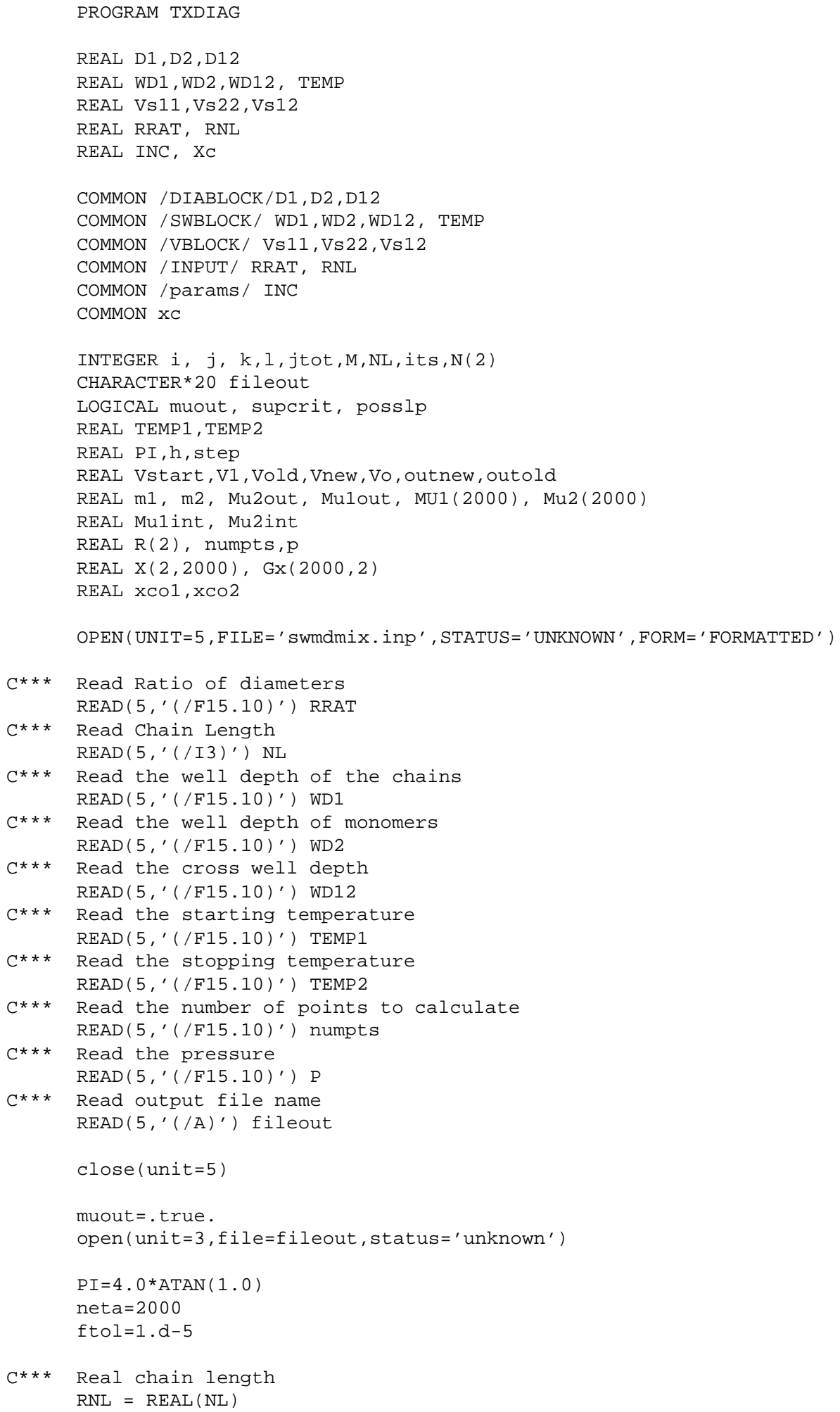




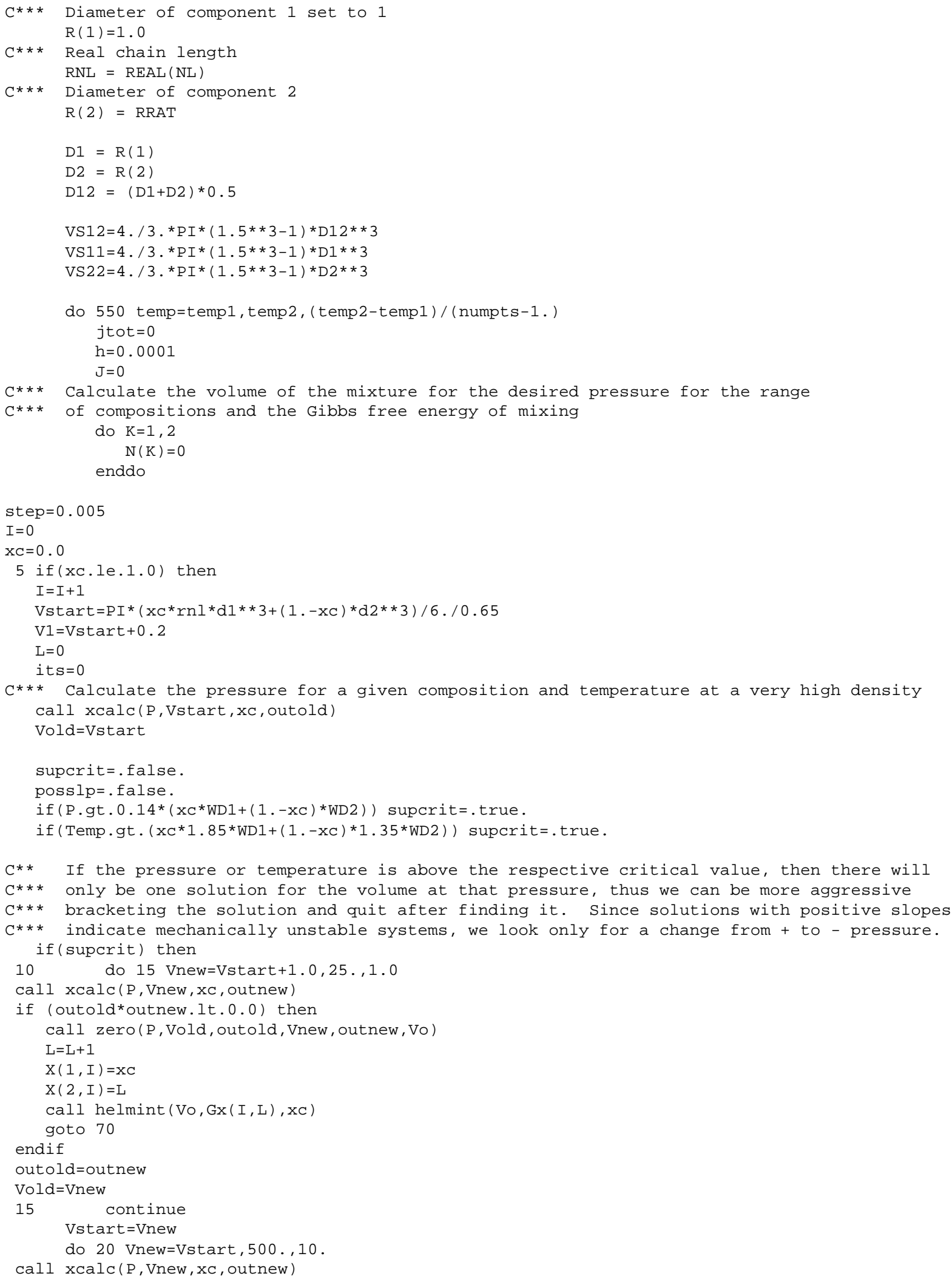




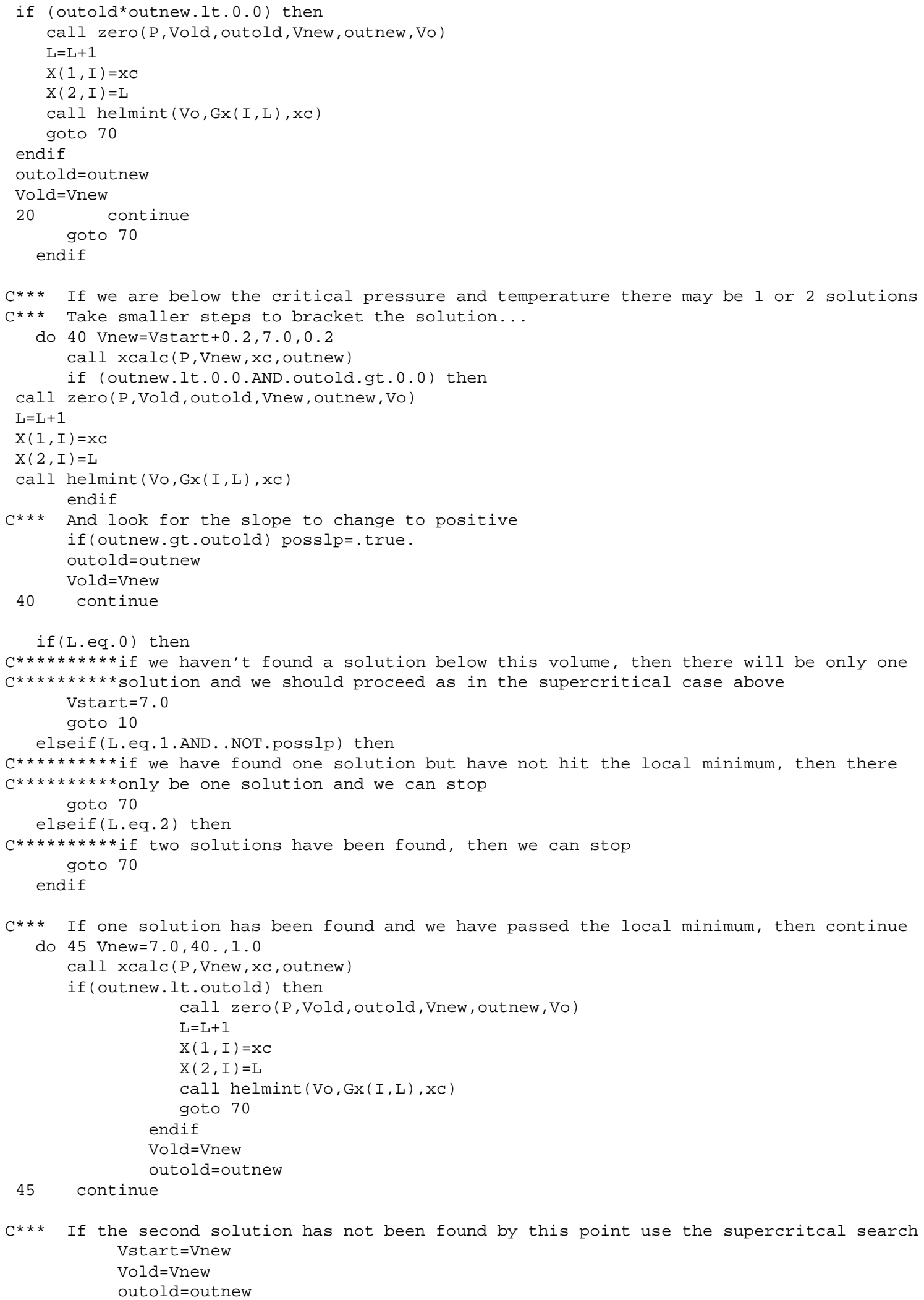




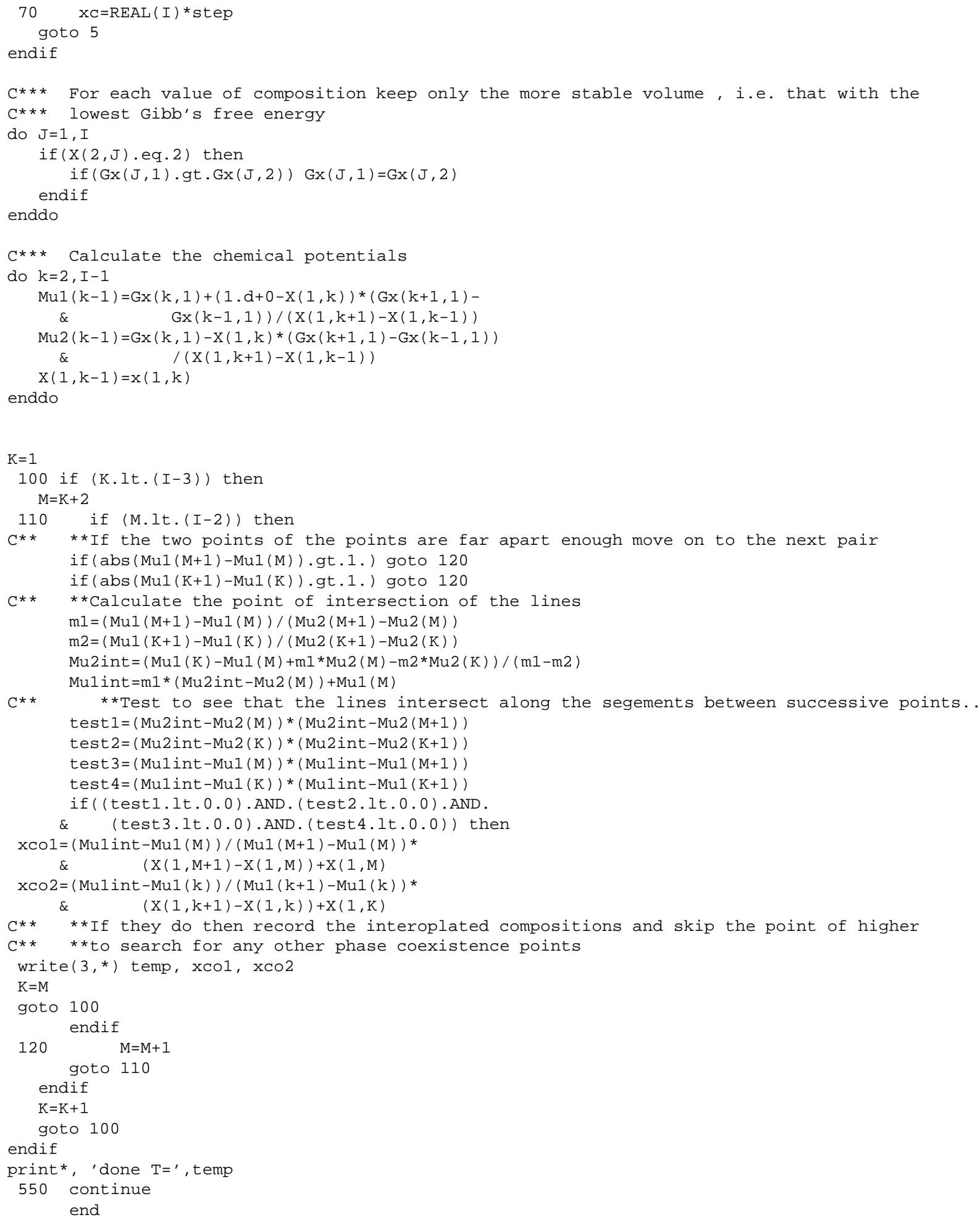


\#include <Monchnhelm.f>

\#include <xcalc.f >

\#include "com_vol_dum.f"

\#include "chn_mon_vol.f"

\#include "subrtns.f"

\#include "zerofind.f" 
SUBROUTINE xcalc $(P, V, x c$, out $)$

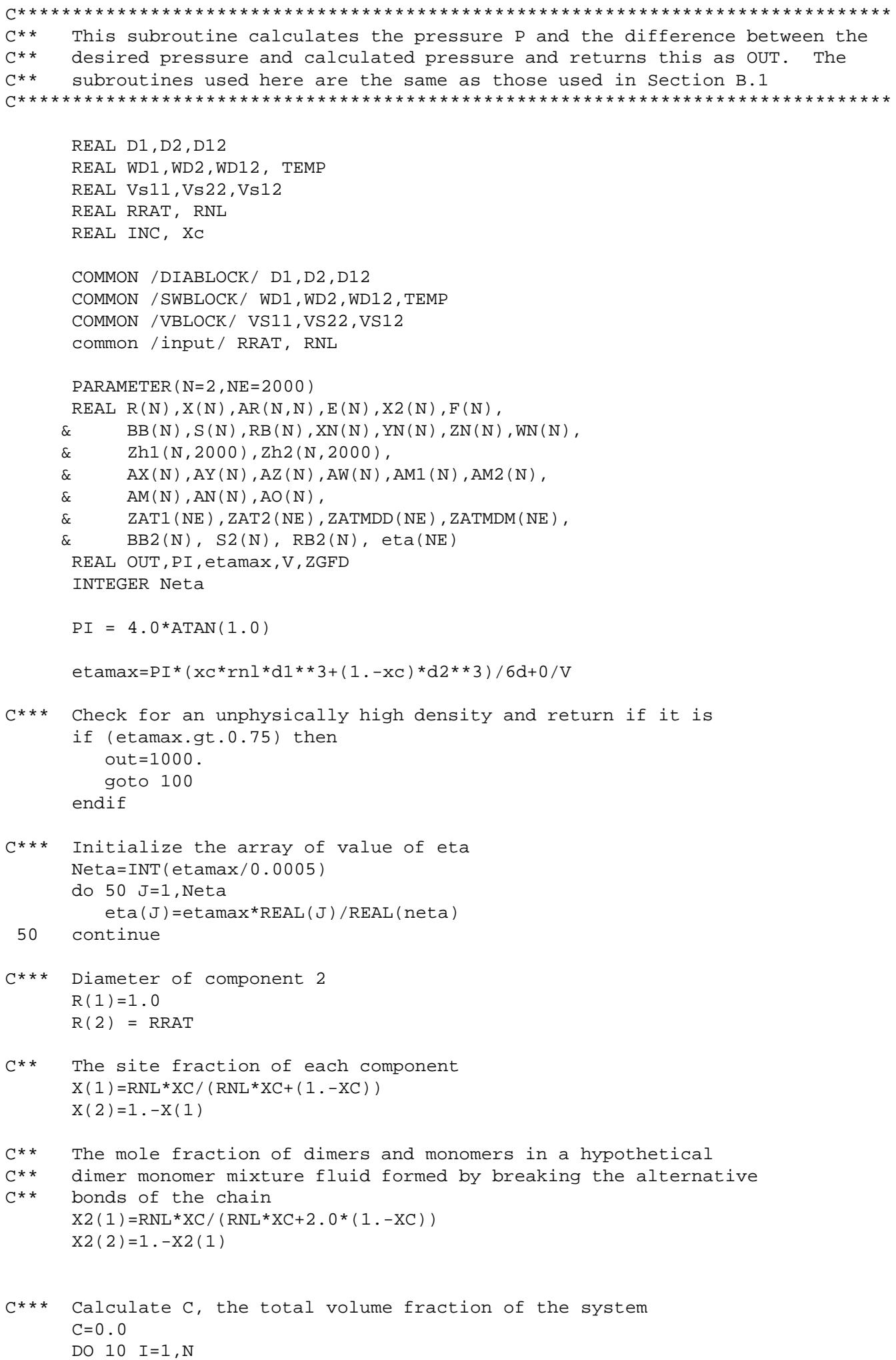




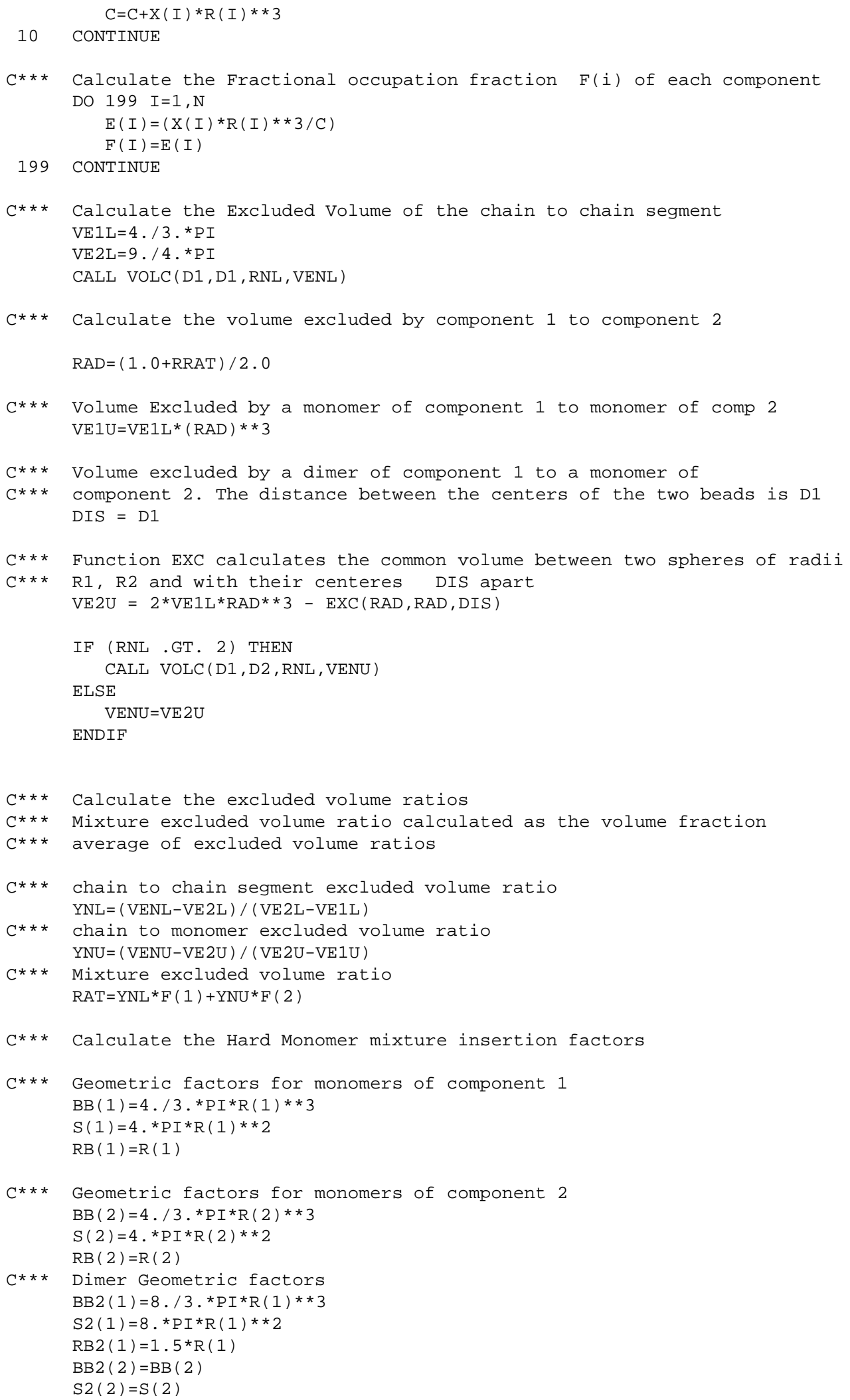


$\operatorname{RB} 2(2)=\operatorname{RB}(2)$

$C * * *$ Calculate the insertion factor for monomer 1 and 2

$\mathrm{C} \star \star \star$ at Eta ranging from $\quad$ Eta $=0.0-->0.5$ Increment $=0.005$

CALL SPTZ (BB, RB, S, X, eta, Zh1, neta)

C*** Calculate the insertion factors for Dimers and Monomers

$C^{* \star *}$ at Eta ranging from Eta $=0.0-->0.5$ Increment $=0.005$

CALL SPTZ (BB2, RB2, S2, X2, eta, Zh2, neta)

C*** Calculate the SW contribution to the compressibility

$C * \star *$ factor from coordination number models.

call monmon (x, eta, zat1, zat2, neta)

call dimmon ( $x 2$, eta, zatmdd, zatmdm, neta)

C*** Calculate the total compressibility factor of the chain monomer

$\mathrm{C} \star \star \star$ mixture.

$\mathrm{ZGFD}=\mathrm{XC} *((\operatorname{RAT}+1.0) *(\mathrm{Zh} 2(1$, neta $)+\mathrm{ZATMDD}($ neta $))-$

$\& \quad \operatorname{RAT}^{*}(\operatorname{Zh} 1(1$, neta $)+\operatorname{ZAT} 1($ neta $\left.))\right)+(1 .-\mathrm{XC})$ *

$\& \quad(\operatorname{Zh} 2(2$, neta $)+\operatorname{ZATMDM}($ neta $))$

Pstar $=\mathrm{ZGFd} * \mathrm{TEMP} / \mathrm{V}$

$\mathrm{OUT}=\mathrm{P}$ star $-\mathrm{P}$

100 return

end 
SUBROUTINE zero(P, Vold, outold, Vnew, outnew, Vo)

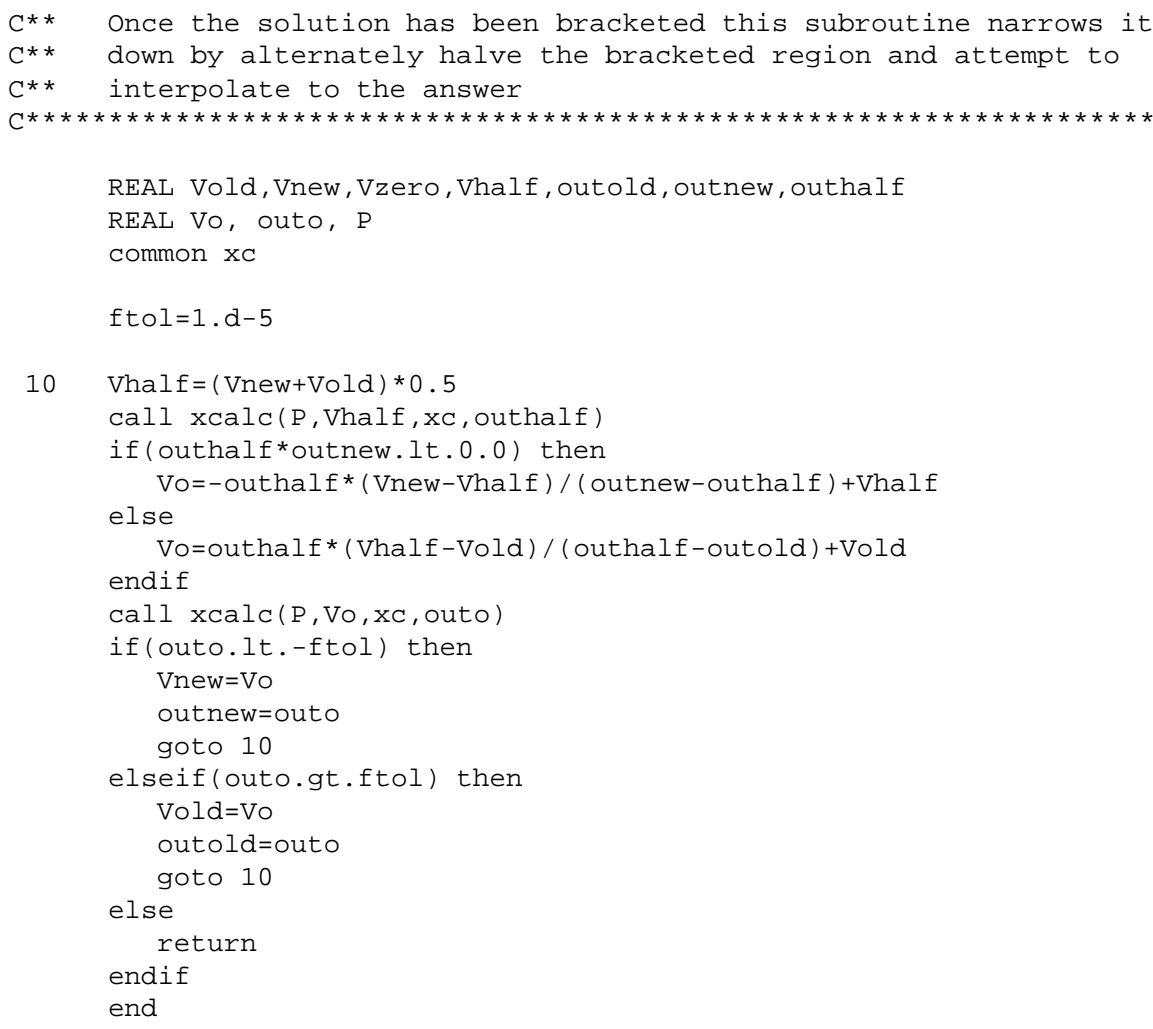


SUBROUTINE helmint (Vout, Gex, xc)

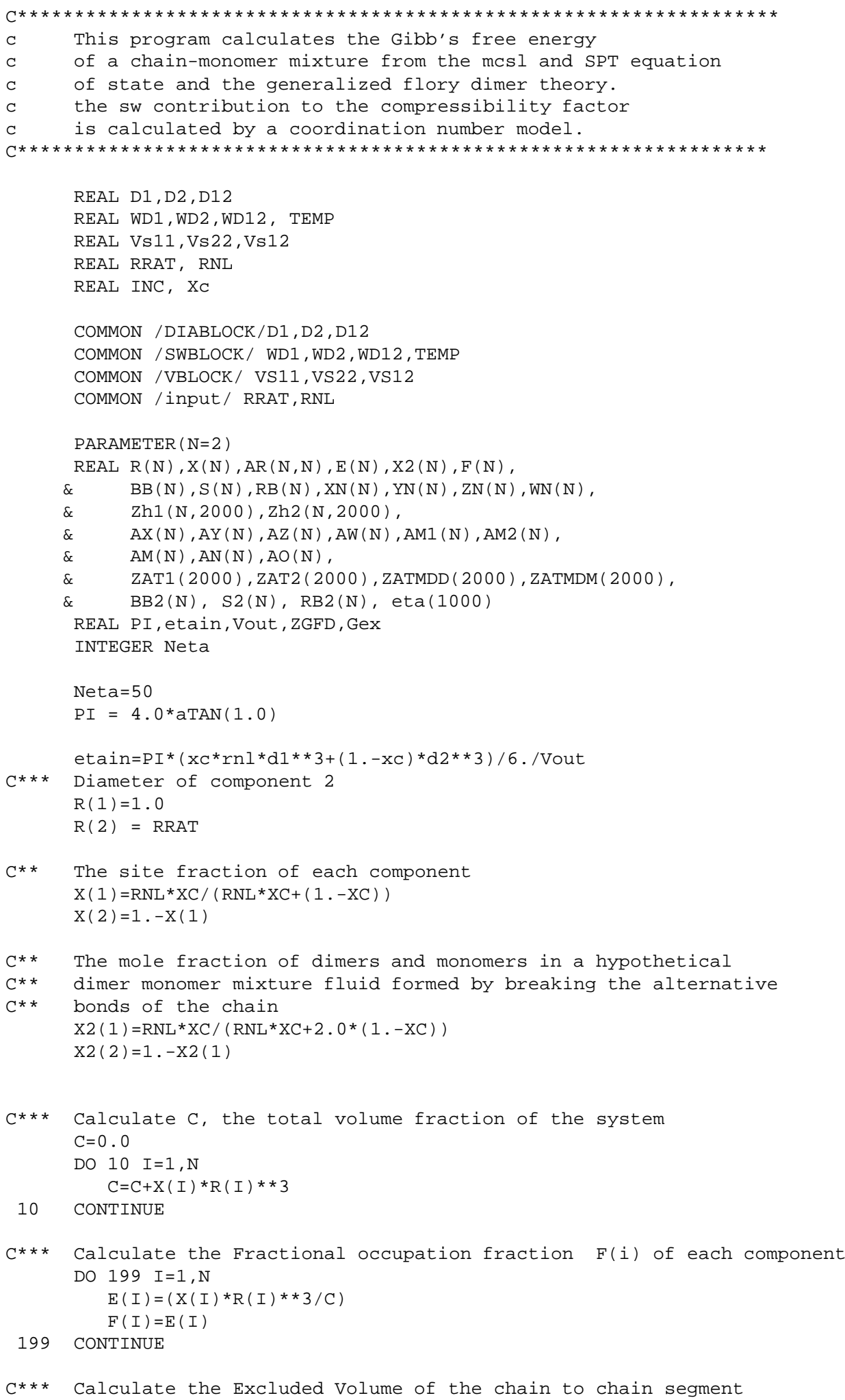


$\mathrm{VE} 1 \mathrm{~L}=4 . / 3 . * \mathrm{PI}$

$\mathrm{VE} 2 \mathrm{~L}=9 . / 4 . * \mathrm{PI}$

CALL VOLC (D1, D1, RNL, VENL)

C*** Calculate the volume excluded by component 1 to component 2

$\operatorname{RAD}=(1.0+\mathrm{RRAT}) / 2.0$

C*** Volume Excluded by a monomer of component 1 to monomer of comp 2

$\mathrm{VE} 1 \mathrm{U}=\mathrm{VE} 1 \mathrm{~L} *(\mathrm{RAD}) * \star 3$

C*** Volume excluded by a dimer of component 1 to a monomer of

$C^{* * *}$ component 2 . The distance between the centers of the two beads is D1 $\mathrm{DIS}=\mathrm{D} 1$

C*** Function EXC calculates the common volume between two spheres of radii

$\mathrm{C} \star \star \star$ R1, R2 and with their centeres DIS apart

$\mathrm{VE} 2 \mathrm{U}=2 * \mathrm{VE} 1 \mathrm{~L} * \mathrm{RAD} * * 3-\mathrm{EXC}(\mathrm{RAD}, \mathrm{RAD}, \mathrm{DIS})$

IF (RNL . GT . 2) THEN

CALL VOLC (D1, D2, RNL, VENU)

ELSE

$\mathrm{VENU}=\mathrm{VE} 2 \mathrm{U}$

ENDIF

$C \star \star \star$ chain to chain segment excluded volume ratio

$\mathrm{YNL}=(\mathrm{VENL}-\mathrm{VE} 2 \mathrm{~L}) /(\mathrm{VE} 2 \mathrm{~L}-\mathrm{VE} 1 \mathrm{~L})$

$\mathrm{C} * * *$ chain to monomer excluded volume ratio

$\mathrm{YNU}=(\mathrm{VENU}-\mathrm{VE} 2 \mathrm{U}) /(\mathrm{VE} 2 \mathrm{U}-\mathrm{VE} 1 \mathrm{U})$

$C \star \star \star$ Mixture excluded volume ratio

$R A T=Y N L * F(1)+Y N U * F(2)$

C*** Calculate the Hard Monomer mixture insertion factors

$C \star \star \star$ Geometric factors for monomers of component 1

$\mathrm{BB}(1)=4 . / 3 . * \mathrm{PI} * \mathrm{R}(1) \star \star 3$

$\mathrm{S}(1)=4 \cdot{ }^{\star} \mathrm{PI} \star \mathrm{R}(1) \star \star 2$

$\mathrm{RB}(1)=\mathrm{R}(1)$

$\mathrm{C} * \star *$ Geometric factors for monomers of component 2

$\mathrm{BB}(2)=4 . / 3 . * \mathrm{PI} * \mathrm{R}(2) \star \star 3$

$\mathrm{S}(2)=4 \cdot{ }^{*} \mathrm{PI} * \mathrm{R}(2) \star \star 2$

$\mathrm{RB}(2)=\mathrm{R}(2)$

$\mathrm{C} \star \star \star \quad$ Dimer Geometric factors

$\mathrm{BB} 2(1)=8 \cdot / 3 . * \mathrm{PI} * \mathrm{R}(1) \star \star 3$

$\mathrm{S} 2(1)=8 . * \mathrm{PI} * \mathrm{R}(1) \star \star 2$

$\mathrm{RB} 2(1)=1.5 * \mathrm{R}(1)$

$\mathrm{BB} 2(2)=\mathrm{BB}(2)$

$\mathrm{S} 2(2)=\mathrm{S}(2)$

$\mathrm{RB} 2(2)=\mathrm{RB}(2)$

C*** Calculate the insertion factor for monomer 1 and 2

$C \star \star *$ at Eta ranging from Eta $=0.0->0.5$ Increment $=0.005$

Neta $=$ INT (etain/0.0005)

do $50 \mathrm{~J}=1$, neta

eta $(J)=$ etain*REAL $(J) / R E A L($ neta)

50 continue

CALL SPTZ (BB, RB, S, X, eta, Zh1, neta)

$\mathrm{C} * * *$ Calculate the insertion factors for Dimers and Monomers

$C^{\star \star *}$ at Eta ranging from Eta $=0.0->0.5$ Increment $=0.005$

CALL SPTZ (BB2, RB2, S2, X2, eta, Zh2, neta)

C*** Calculate the SW contribution to the compressibility

C*** factor from coordination number models. 


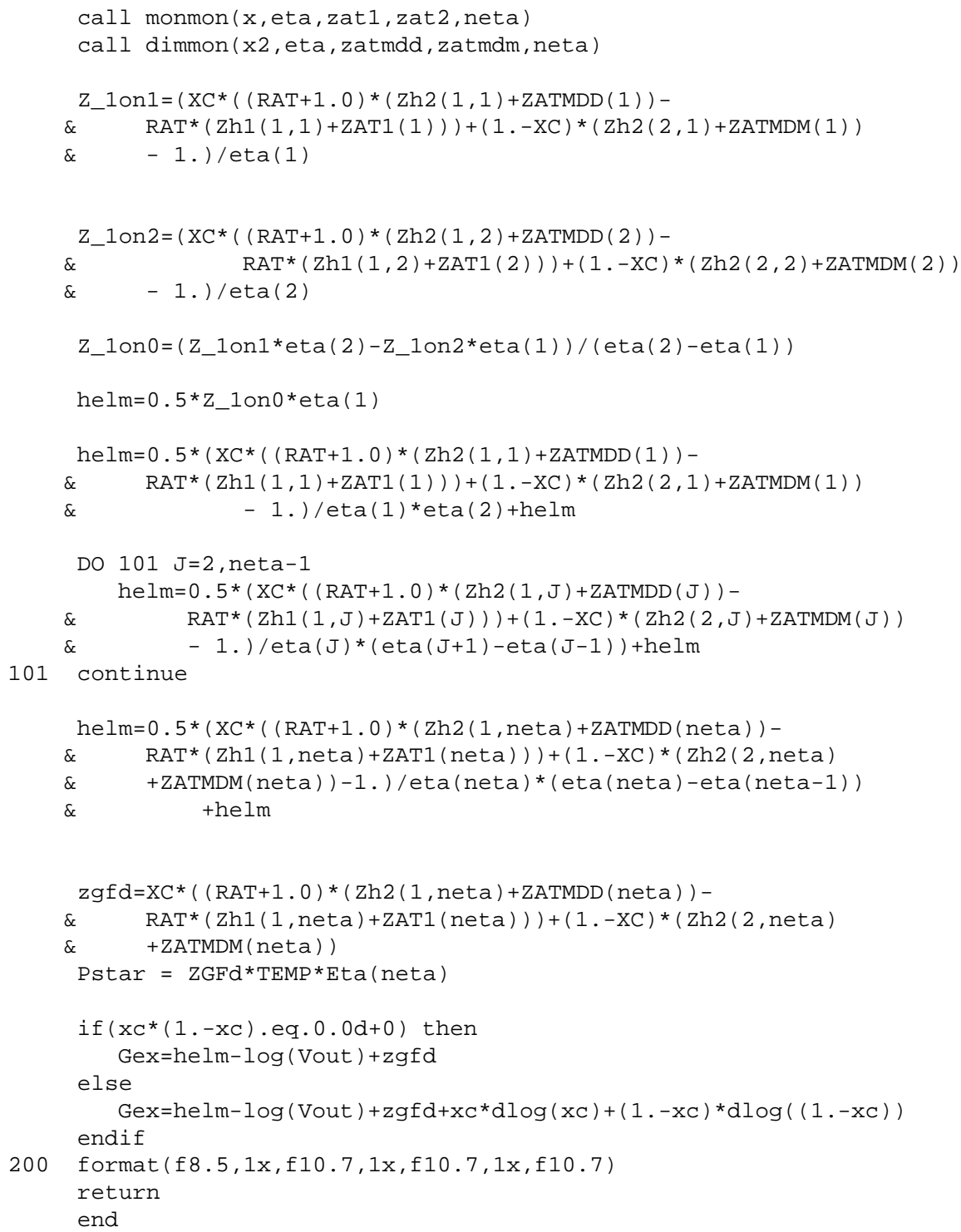

